Subscriber access provided by The Bodleian Libraries of the University of Oxford

\title{
Article
}

\section{Secondary products from intramolecular cycloadditions of azidoalkyl enol ethers and azidoalkyl vinyl bromides. 1-Azadienes, their reactions with diphenylketene, and radical cyclizations to form bi- and tricyclic lactams}

\author{
John T. R. Liddon, Peter J. Lindsay-Scott, and Jeremy Robertson
}

J. Org. Chem., Just Accepted Manuscript • DOI: 10.1021/acs.joc.9b02005 • Publication Date (Web): 23 Sep 2019

Downloaded from pubs.acs.org on September 24, 2019

\section{Just Accepted}

\begin{abstract}
"Just Accepted" manuscripts have been peer-reviewed and accepted for publication. They are posted online prior to technical editing, formatting for publication and author proofing. The American Chemical Society provides "Just Accepted" as a service to the research community to expedite the dissemination of scientific material as soon as possible after acceptance. "Just Accepted" manuscripts appear in full in PDF format accompanied by an HTML abstract. "Just Accepted" manuscripts have been fully peer reviewed, but should not be considered the official version of record. They are citable by the Digital Object Identifier (DOI®). "Just Accepted" is an optional service offered to authors. Therefore, the "Just Accepted" Web site may not include all articles that will be published in the journal. After a manuscript is technically edited and formatted, it will be removed from the "Just Accepted" Web site and published as an ASAP article. Note that technical editing may introduce minor changes to the manuscript text and/or graphics which could affect content, and all legal disclaimers and ethical guidelines that apply to the journal pertain. ACS cannot be held responsible for errors or consequences arising from the use of information contained in these "Just Accepted" manuscripts.
\end{abstract}




\title{
Secondary products from intramolecular cycloadditions of azidoalkyl enol ethers and azidoalkyl vinyl bromides. 1-Azadienes, their reactions with diphenylketene, and radical cyclizations to form bi- and tricyclic lactams.
}

John T. R. Liddon, ${ }^{\dagger}$ Peter J. Lindsay-Scott, ${ }^{\star}$ and Jeremy Robertson $*, \dagger$

$\dagger$ Department of Chemistry, University of Oxford, Chemistry Research Laboratory, Mansfield Road, Oxford, OX1 3TA, UK.

* Eli Lilly and Company Limited, Erl Wood Manor, Windlesham, Surrey, GU20 6PH, UK.

TABLE OF CONTENTS GRAPHIC

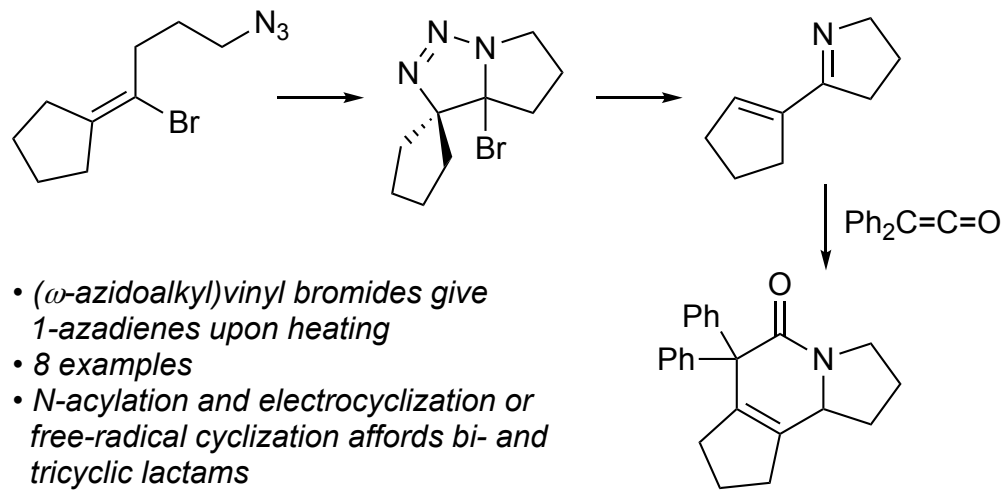

\begin{abstract}
Azidoalkyl enol ethers undergo intramolecular 1,3-dipolar cycloaddition to generate stable triazolines; in contrast, the cycloadducts formed by heating analogous azidoalkyl vinyl bromides are unstable with respect to elimination of $\mathrm{N}_{2}$ and $\mathrm{HBr}$, affording 1-azadienes (2-alkenyl cyclic imines). These primary products may be isolated or treated directly with diphenylketene to produce bi- and tri-cyclic 3,4-dihydropyridin-2(1H)-ones; similar ring systems may also be produced from the azadienes by $N$-acylation and radical cyclization.
\end{abstract}

\section{INTRODUCTION}

Within a general research program aiming to develop new methods for assembling multicyclic alkaloids, we speculated that triazolium ions $\mathbf{5}$ (Scheme 1 ), formed by hypothetical protonation of triazolines $\mathbf{2}$, might enter into potentially valuable chemistry centered on the addition of carbon-based nucleophiles either directly or following 
alkyl shift $(\rightarrow 6)$. Loss of nitrogen from the triazolium ions was expected to be unfavourable, in view of the likely high energy of the so-formed 1-azaallyl cations 10. ${ }^{1}$ The intramolecular dipolar cycloaddition of (2azidoethyl)allenes 1 (Scheme 1A) is an unsuitable route to the precursor triazolines $\mathbf{2}$ because the cycloadducts lose nitrogen directly to generate azatrimethylenemethane (ATMM) diyls $\mathbf{3}$ which, in turn, evolve into alkenyl isocyanides 4 unless tethered alkene or diene functionality is present to trap them rapidly. ${ }^{2}$ The same triazolium ions 5 could potentially be generated by Lewis-acid mediated abstraction of $X^{-}$from triazolines 8 (Scheme 1B), formed by intramolecular azide cycloaddition onto a suitable heteroatom-bearing alkene $7 .^{3}$ It was appreciated at the outset that triazolines $\mathbf{8}$ might, under the reaction conditions used for their formation, be susceptible to loss of nitrogen and migration of the X-group giving pyrrolines 9 . This paper summarizes the results of an evaluation of this approach, and selected transformations of the 1-azadienes produced from vinyl bromides 7 (X = Br) via pyrrolines 9 or equivalent species.

A

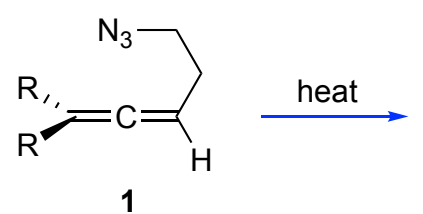

B

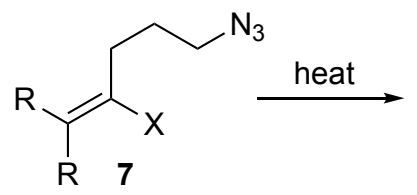

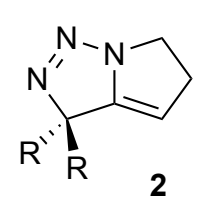

2
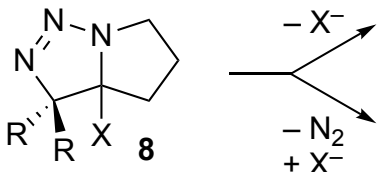

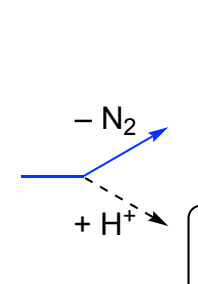

Ri

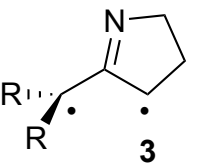

3
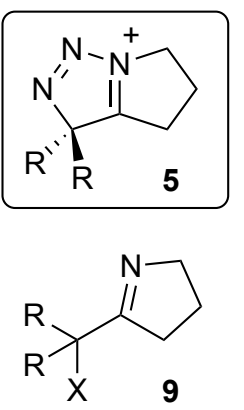
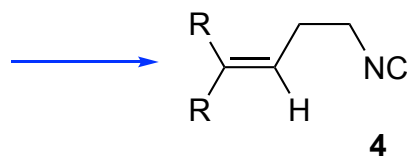

4
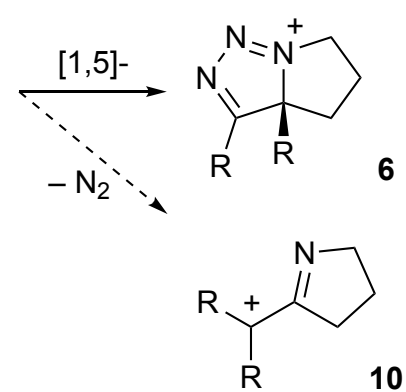

Scheme 1 A: Cycloaddition of (2-azidoethyl)allenes 1 leads to $\gamma, \delta$-unsaturated isocyanides 4; B: Cycloaddition of heteroatom-substituted ( $\omega$-azidoalkyl)alkenes $\mathbf{7}$ as a potential route to triazolium ions $\mathbf{5}$ and $\mathbf{6}$.

\section{RESULTS AND DISCUSSION}

\section{Enol ethers}

With some limited precedent available on intramolecular azide + enol ether cycloadditions to give isolable adducts $^{4,5}$ an initial exploration of this idea focused on substrates 11-13 (Figure 1), easily prepared by deprotonation and alkylation ${ }^{6}$ of ethyl vinyl ether, dihydrofuran, dihydropyran, or furan (Scheme S1). In general, the rate of azide + alkene cycloaddition varies dramatically with, inter alia, variation of the electronic character of the alkene component. ${ }^{7}$ For example, the second order rate constant for the reaction of azidobenzene with $N$ (cyclopenten-1-yl)pyrrolidine is around $235,000 \times$ larger than that for the reaction with 1-ethoxycyclopentene; ${ }^{8}$ accordingly, it was expected that the rate of reaction of substrates 11-13 would be low. In practice, heating the substrates in toluene at $130{ }^{\circ} \mathrm{C}$ (pressure tube), with $\mathrm{K}_{2} \mathrm{CO}_{3}$ present to minimize acid-mediated decomposition pathways, led to essentially complete conversion to bi- and tri-cyclic triazolines 14 and 15 over a period of 2-4 days, accompanied, in the reactions of 11 , by variable quantities of the triazoles $16^{9}$ and $17^{9,10}$ resulting from elimination of ethanol. The cycloadditions of the azidobutyl substrates $\mathbf{1 1} \mathbf{b}$ and $\mathbf{1 2 b}$ were somewhat slower than those of the corresponding azidopropyl substrates $\mathbf{1 1 a}$ and 12a, in line with a less unfavourable entropy of 
activation in the latter pair. The dihydrofuranyl substrates $\mathbf{1 2} \mathbf{a}, \mathbf{b}$ reacted more rapidly than their simpler ethoxy counterparts 11, presumably as a result of slight strain-activation. This advantage is absent in the dihydropyranyl substrate 12c which, in combination with the longer linking chain, required 10 days for full consumption of the substrate, and a crude product of poor quality was obtained from which only a $20 \%$ yield of cycloadduct $15 \mathrm{c}$ was isolated. The furanyl substrate $13^{11}$ merely decomposed unproductively at temperatures up to $170^{\circ} \mathrm{C}$.

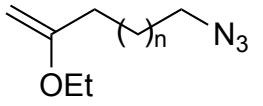

$11 a, n=1$

$11 \mathrm{~b}, \mathrm{n}=2$

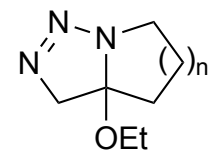

$14 a, n=1$

$14 b, n=2$

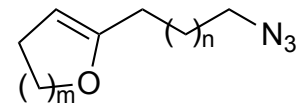

12a, $m, n=1$

$12 b, m=1, n=2$

$12 c, m, n=2$

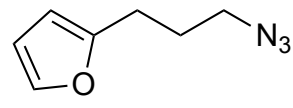

13

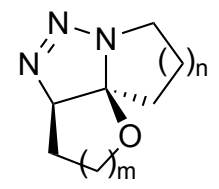

15a, $\mathrm{m}, \mathrm{n}=1$

$15 b, m=1, n=2$

$15 c, m, n=2$

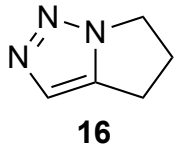<smiles>c1nnn2c1CCCC2</smiles>

17

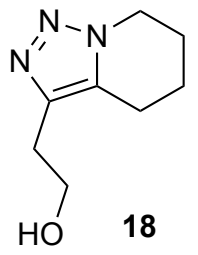

18

Figure 1 ( $\omega$-Azidoalkyl)enol ethers (11-13), the products of their intramolecular cycloaddition $(\mathbf{1 4}, \mathbf{1 5})$, and triazoles formed by elimination (16-18).

Attempts to generate and trap iminium intermediates from hemiaminals $\mathbf{1 4}$ and $\mathbf{1 5 b}$ resulted either in recovery of starting material (e.g. EtMgBr, $-78{ }^{\circ} \mathrm{C} \rightarrow \mathrm{RT}$; or $\mathrm{Et}_{3} \mathrm{Al}$ ) or aromatization ( $\rightarrow$ 16-18; e.g. EtMgBr, THF, reflux; or allyltributylstannane + a variety of Lewis acids), with nucleophilic addition being unable to compete with rapid proton loss. In order to shut down the aromatization pathway, significant effort was expended on a search for an effective general route to substrates bearing two alkyl substituents at the distal end of the alkene. Eventually, a small, impure sample of cyclohexylidene substrate 19 was prepared via Johnson-Claisen rearrangement of the adduct of lithiated ethyl vinyl ether and cyclohexanone (Scheme S2). ${ }^{12}$ With sufficient enol ether available for just a single experiment, the thermolysis was conducted in toluene- $d_{8}$ and the reaction progress was followed by NMR analysis. After 3.5 days at $130^{\circ} \mathrm{C}$, the major product of the reaction was assigned as pyrroline derivative $\mathbf{2 1}$ arising from overall loss of nitrogen and migration of the alkoxy group (Scheme 2; $c f .9$, Scheme 1). It appeared from this result that the combination of steric crowding and relatively favourable stabilization of any developing positive charge rendered the intermediate cycloadduct 20 unstable under the conditions of the reaction and, coupled with the difficulty of preparing suitable substrates, investigations on azidoalkyl enol ether cycloadditions were terminated. 


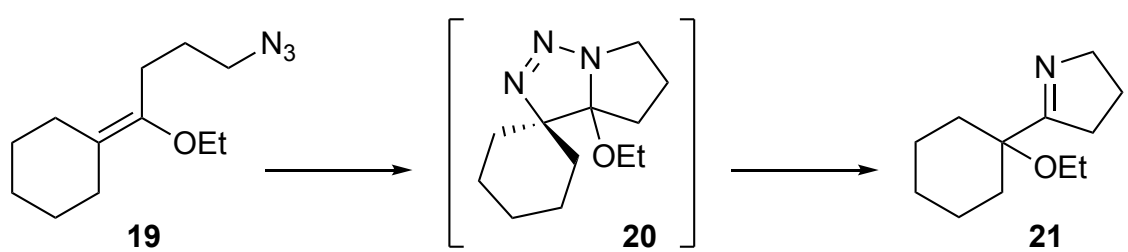

Scheme 2 Intramolecular azide + alkene cycloaddition of fully-substituted enol ether 19 with loss of $\mathrm{N}_{2}$ and accompanying 1,2-ethoxy-group shift.

\section{Vinyl bromides}

Without an efficient, general route available to fully-substituted enol ethers, focus switched to vinyl bromides since their preparation by bromination/dehydrobromination of the corresponding alkenes is straightforward. ${ }^{13}$ Little is reported on the non-catalysed, purely thermal, cycloaddition of azides and simple vinyl bromides ${ }^{14}$ and, to the best of our knowledge, intramolecular azide + vinyl bromide cycloadditions have not been studied. The results would therefore provide a comparison with analogous enol ethers as well as potentially providing access to triazolium ions as outlined in Scheme 1B.

Substrates 22a-f,i (Figure 2, preparation: Schemes S3-S7) were chosen so as to provide meaningful comparison with the enol ethers already studied, and to probe the effect of structural variations in the substrate (tether length, electronic and strain effects on the alkene, conformational restriction). The hydroxy- and azido-methyl substrates $\mathbf{2 2} \mathbf{g}$, $\mathbf{h}$ were prepared in order to gain information on functional group compatibility with the cycloaddition conditions and to introduce an intermediate level of conformational restriction compared with the un- and gemdimethyl-substituted substrates 22a and 22f, respectively.
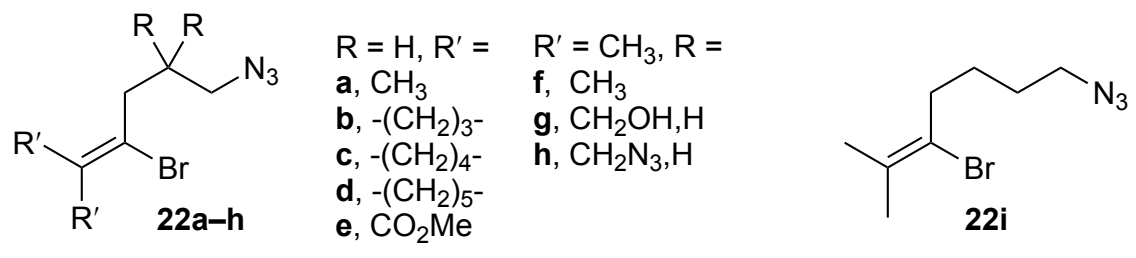

Figure 2 ( $\omega$-Azidoalkyl)vinyl bromide substrates.

A preliminary study of the reactivity of azidoalkyl vinyl bromides, using substrate 22a, showed that the choice of solvent ${ }^{15}$ (between available high-boiling deuterated solvents for an NMR study: DMSO- $d_{6}$, pyridine- $d_{5}$, toluene- $d_{8}$ ) had a merely marginal effect on reaction rate and, for ease of product isolation, toluene was chosen as in the enol ether work. Running the reactions in dilute solution (0.05-0.1 M) minimized the production of oligomeric products, and the formation of tarry deposits during the reaction was reduced by including a solid base to sequester the liberated $\mathrm{HBr}$ (see below), with a combination of $\mathrm{K}_{2} \mathrm{CO}_{3}$ (10.0 equiv.) and $\mathrm{NaOH}$ (2.0 equiv.) providing the cleanest product mixtures. Azide 22a converted into products very slowly at $100{ }^{\circ} \mathrm{C}$ but at $150{ }^{\circ} \mathrm{C}$ the reaction progressed steadily and was complete after around $40 \mathrm{~h}$. These reaction parameters were then employed in the thermolysis 
of the remaining substrates $\mathbf{2 2} \mathbf{b}-\mathbf{i}$, the corresponding products of which are shown in Figure 3 which also provides the approximate reaction times required for the starting material to be consumed (TLC).

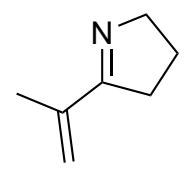

23a, $73 \%(44 \mathrm{~h})$

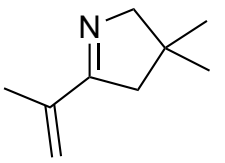

23f, $71 \%(24 \mathrm{~h})$

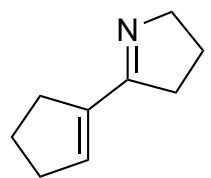

23c, $78 \%(48 \mathrm{~h})$

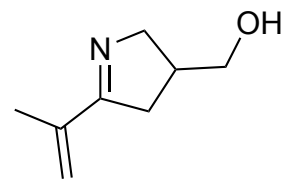

$23 g, 82 \%(27 \mathrm{~h})$

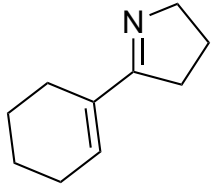

23d, $84 \%(30 \mathrm{~h})$

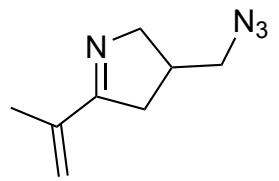

$23 \mathrm{~h}, 76 \%(27 \mathrm{~h})$<smiles>COCC(C(=O)OC)=C1CCCN1</smiles>

$24 \mathrm{e}, 20 \%^{\mathrm{a}}$

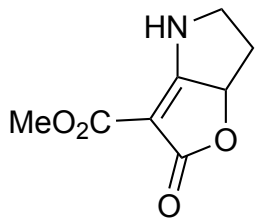

25e, $60 \%(11 \mathrm{~h})^{\mathrm{a}}$

Figure 3 Products formed from the thermolysis of azidopropyl vinyl bromides 22a-i. Reaction conditions: toluene, $\mathrm{K}_{2} \mathrm{CO}_{3}, \mathrm{NaOH}, 150{ }^{\circ} \mathrm{C}$ (pressure tube). Percentage yields refer to products isolated following column chromatography. The products from $\mathbf{2 2} \mathbf{b}$ are shown in Scheme $3 .{ }^{a}$ Reaction temperature $=100{ }^{\circ} \mathrm{C}$.

In all cases, the reaction temperature and time combined to ensure that the primary cycloadducts $(8, \mathrm{X}=\mathrm{Br}$, Scheme $1 \mathrm{~B}$ ) ejected $\mathrm{N}_{2}$ and $\mathrm{HBr}$ to generate cyclic imines 23 in all cases, except where noted below. The relative rates of reactions of homologous substrates $\mathbf{2 2} \mathbf{b}-\mathbf{d}$ roughly parallel the rates of $\mathrm{NaBH}_{4}$ reduction of the analogous ketones, ${ }^{16}$ reflecting differential contributions of angle strain at the non-brominated alkene carbon and significant rehybridization at that center in the cycloaddition transition state. The cyclobutyl substrate $\mathbf{2 2} \mathbf{b}$ reacted rapidly to give a complex product mixture from which three components $(\mathbf{2 7}, \mathbf{2 9}, \mathbf{3 0}$, Scheme 3 ) were isolated and characterized. These products gave some insight into the nature of the reactions to form azadienes $\mathbf{2 3}$, in particular the potential involvement of a cationic intermediate $(\mathbf{2 8} ; c f . \mathbf{1 0}$, Scheme 1$)$ en route to the azadienes. A fourth component, observed as a contaminant in some samples of alcohol $\mathbf{3 0}$ and not isolated as a pure compound, is tentatively assigned as cyclohexene derivative $\mathbf{3 2}$ on the basis of NMR spectroscopic and HRMS data; this is presumed to arise by electrocyclic ring-opening of intermediate $\mathbf{2 3 \mathbf { b }}$ and Diels-Alder dimerization.

As expected, the presence of the gem-dimethyl substituent in substrate $\mathbf{2 2 f}$ led to a significant acceleration of the reaction, ${ }^{17}$ supporting a rate-limiting cycloaddition step; even mono-substitution of the linking chain (in $\mathbf{2 2 g}$, h) led to an appreciably reduced reaction time compared to the unsubstituted analogue. Conversely, the extra conformational mobility offered by the additional methylene group linking the azide and vinyl bromide functionality in 22i led to a dramatic reduction in the rate of reaction; various decomposition pathways became significant during the extended reaction time, lowering the yield of the azadiene $\mathbf{2 3 i}$. 

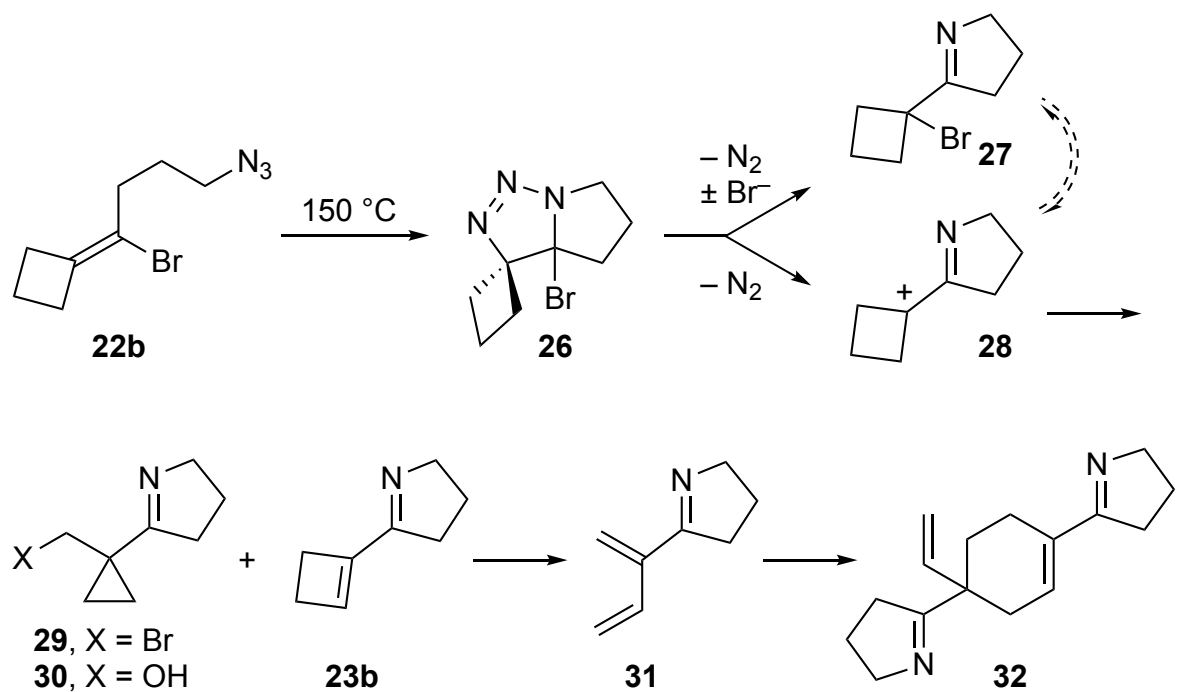

Scheme 3 Minor products $\mathbf{2 7}, \mathbf{2 9}, \mathbf{3 0}$, and $\mathbf{3 2}$ generated from the cyclobutylidene substrate $\mathbf{2 2 b}$.

The malonyl derivative 22e was prepared in the expectation that the electron-deficient alkene would react rapidly, possibly at a sufficiently low temperature to allow the primary cycloadduct $\mathbf{3 3}$ (Scheme 4) to be isolated, but also to block the final elimination reaction, potentially giving insight into the nature of the intermediates in the overall transformation. In the event, the reaction was complete within $11 \mathrm{~h}$ at $100^{\circ} \mathrm{C}$ (cf. $\sim 7 \%$ conversion of 22a after 89 $\mathrm{h}$ at $100^{\circ} \mathrm{C}$ ) but, again, the cycloadduct 33 was not isolated under these conditions. Accepting that any discussion of the mechanism leading to the obtained products $24 \mathrm{e}^{18}$ and $25 \mathrm{e}$ is purely speculative, it is conceivable that the major product 25e arises from bromoimine intermediate 34 via tautomerization $(\rightarrow 35)$ and a process formally equivalent to an oxapentadienyl cation electrocyclization. ${ }^{19}$ The minor component $\mathbf{2 4 e}$ could arise from the same bromoimine intermediate by providing $\mathrm{Br}^{+}$to a suitable nucleophile present in the reaction mixture (pathway $\mathbf{B}$ ) or, for example, by homolytic loss of $\mathrm{N}_{2}$ and $\mathrm{Br}^{\bullet}(\rightarrow 36)$ then abstraction of a hydrogen atom (e.g. from the solvent) (pathway A). Compounds with the tetrahydrofuro[3,2-b]pyrrol-2-one core present in product 25e are known, and generally prepared by intramolecular Wittig reaction of succinimide derivatives, ${ }^{20}$ but $25 e$ appears to be the first reported $\mathrm{N}$-unsubstituted example.<smiles>COC(=O)C(Br)(C(=O)OC)C1=CCCN1</smiles>

33

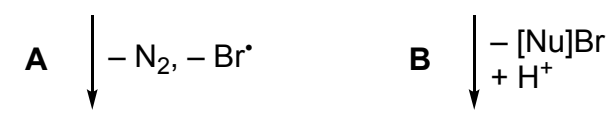<smiles></smiles>

36<smiles>COC(=O)C(C(C)=O)=C1CCCN1</smiles>

24e
35<smiles>[3H][13CH2][13Br]</smiles><smiles>CC(=O)C1=C2NCCC2OC1=O</smiles>

Scheme 4 Outline reaction pathways leading from primary cycloadduct 33 to enamides 24 e and 25 e. 


\section{1-Azadiene reactions}

With two exceptions ( $\mathbf{2 3} \mathbf{d}^{21}$ and $\mathbf{2 3 \mathbf { i } ^ { 2 2 }}$ ) the azadiene products are not reported in the literature and a survey of their reactivity ${ }^{23}$ was undertaken. ${ }^{24}$ Two aspects of this survey shall be described here, with the linking feature being the formation of 1-azabicyclo[4.3.0]nonane derivatives, the core ring system in, for example, the large class of indolizidine alkaloids. ${ }^{25}$ First, it was found that azadiene 23a showed little reactivity as a diene in attempted DielsAlder cycloadditions with both electron-deficient and electron-rich potential dienophiles; however, an equivalent transformation proceeded efficiently upon treatment with diphenylketene. ${ }^{26}$ For convenience, the reaction mixtures obtained from heating azides $\mathbf{2 2}$ were simply cooled to RT and diphenylketene added directly; the intermediate azadienes $\mathbf{2 3}$ reacted efficiently over a period of a few hours to generate the lactams shown in Figure 4. The isolated yields for this one-pot process were generally comparable to the yields obtained for the conversion of azides $\mathbf{2 2}$ to azadienes $\mathbf{2 3}$.

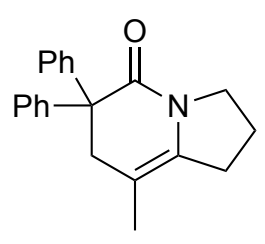

$37 a, 80 \%$

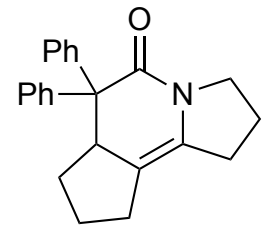

37c, $77 \%$

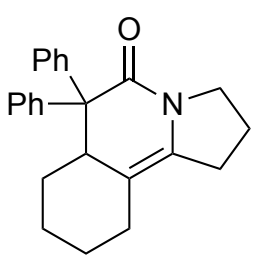

$37 d, 80 \%$

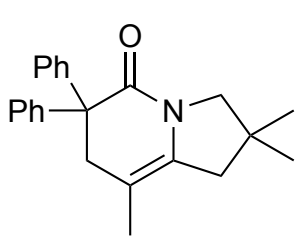

37f, $91 \%$

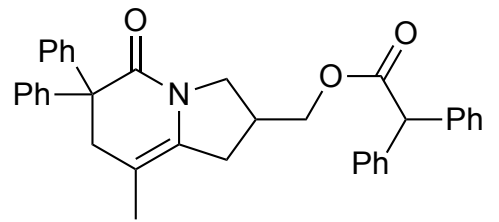

$37 g, 74 \%$

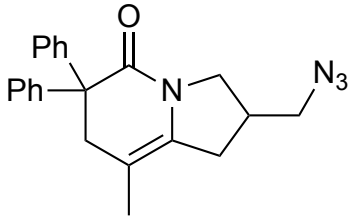

$37 \mathrm{~h}, 58 \%$

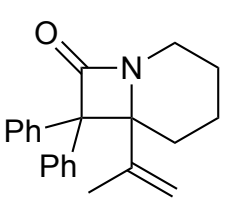

$38 \mathrm{i}, 35 \%$

Figure 4 Products formed, and yields of purified product, from the one-pot thermolysis and subsequent trapping of azidoalkyl vinyl bromides 22. Reaction conditions: toluene, $\mathrm{K}_{2} \mathrm{CO}_{3}, \mathrm{NaOH}, 150{ }^{\circ} \mathrm{C}$ (pressure tube) then $\mathrm{Ph}_{2} \mathrm{C}=\mathrm{C}=\mathrm{O}$, RT.

From Figure 4, the production of $\beta$-lactam 38i appears anomalous; however, the few analogous reported reactions of this type, that concern only acyclic azadienes ${ }^{27}$ tend to generate $\beta$-lactams kinetically which, upon heating, may rearrange to the $\delta$-lactam isomers. Thus, in fact, the production of $\delta$-lactams $\mathbf{3 7}$ at RT from the pyrroline azadienes deserves comment. On the basis that the reactions proceed via a stepwise acylation/(formal) electrocyclization process, ${ }^{27 a}$ the balance in favour of $\delta$-lactam formation from zwitterion 39 (Scheme 5) may reflect the relative resistance to introduce a further $\mathrm{sp}^{3}$-center into the five-membered heterocycle when compared to the ease with which six-membered rings accommodate full saturation. The production of $\delta$-lactam products (from $39, n=1$ ) maintains an $\mathrm{sp}^{2}$-carbon adjacent to the nitrogen throughout the reactions with diphenylketene, whereas $\beta$-lactam formation would necessarily increase unfavourable eclipsing interactions in the transition state for formal electrocyclization. 


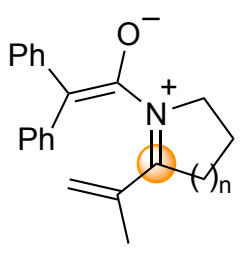

39

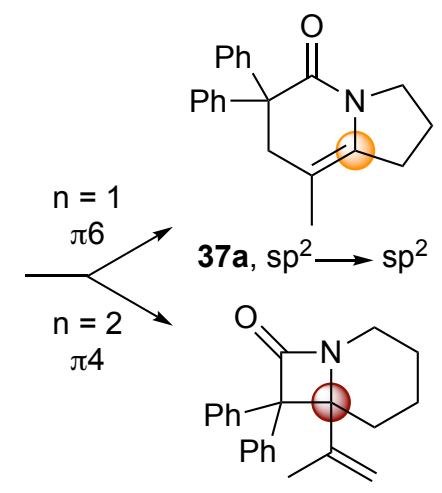

$38 \mathbf{i}, \mathrm{sp}^{2} \longrightarrow \mathrm{sp}^{3}$

Scheme 5 Divergent formal electrocylization modes from pyrroline- $(n=1)$ and piperideine- $(n=2)$ derived zwitterions.

The nucleophilicity of the azadienes $\mathbf{2 3}$ was harnessed for the production, by $\mathrm{N}$-acylation, ${ }^{28}$ of precursors for potential 6-endo-trig radical cyclization, the aim being to produce $\delta$-lactams of the same form as those shown in Figure 4 but with potentially more flexibility. In a proof-of-principle study, $\alpha$-bromoenamide $\mathbf{4 0}$ (Scheme 6), readily prepared from azadiene 23d, was employed in three sets of conditions established for radical cyclization. First, classical reductive cyclization with the tributyltin hydride/AIBN combination produced a mixture of alkene regioisomers $\mathbf{4 1}$ and $\mathbf{4 2}$ arising from $\mathrm{H}$-atom trapping at the termini of the allylic radical formed upon cyclization. However, under $\mathrm{Cu}(\mathrm{I})$-mediated atom-transfer radical cyclization (ATRC) conditions, ${ }^{29}$ using PMDTA as a tridentate ligand for the copper center, ${ }^{30}$ the fragile allylic bromide $\mathbf{4 3}$ was isolated in excellent yield provided that chromatographic purification was carried out rapidly through a short silica plug. When the reaction was conducted with bidentate TMEDA employed as ligand, ${ }^{31}$ slightly more forcing reaction conditions were necessary and diene 44 was obtained cleanly; here, chromatography required basic alumina in order to retain the integrity of the 2amido-1,3-diene motif as the use of silica gel as column support led to diene isomerization, generating a roughly equimolar mixture of isomers $\mathbf{4 4}$ and $\mathbf{4 5}$. 
(a)<smiles>CC1(C)C(=O)N2CCCCC2C2C(=C3CCCCC31)C1[CH+]CCN1C(=O)C2(C)C</smiles>

(b)<smiles>CC1(C)C(=O)N2CCC(Br)C2=C2CCCCC21</smiles>

(c)<smiles>CC1CCCC2=C1C(C)(C)C(=O)N1CCC=C21</smiles>

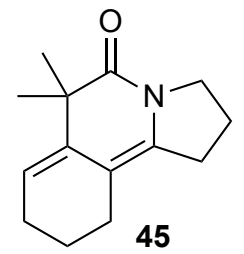

Scheme 6 Radical cyclizations from $\mathrm{N}$-( $\alpha$-bromoisobutyroyl)pyrroline 40 . Reagents and conditions: (a) $\mathrm{Bu}_{3} \mathrm{SnH}$, AIBN, toluene, $110{ }^{\circ} \mathrm{C}, 22 \mathrm{~h}$ (72\%); (b) $\mathrm{CuBr}$ (30 mol\%), PMDTA, $\mathrm{CH}_{2} \mathrm{Cl}_{2}, \mathrm{RT}, 0.25 \mathrm{~h}$ (90\%); (c) $\mathrm{CuBr}$ (110 mol\%), TMEDA, $\mathrm{CH}_{2} \mathrm{Cl}_{2}$, RT $40{ }^{\circ} \mathrm{C}, 2.5 \mathrm{~h}(44,77 \%$ [92\% based on $16 \%$ recovered starting material]).

\section{CONCLUSION}

This work originated from our group's interest in accessing azide/allene cycloadducts 2 (Scheme 1), expected to participate in diverse mechanistic pathways depending on the nature of subsequent reaction conditions and reagents added in situ. The isolable azide/enol ether cycloadducts $8(X=O R)$ are potentially equivalent to cycloadducts $\mathbf{2}$ but these merely aromatized when induced to form derived iminium ions $\mathbf{5}$. In substrates such as 19, whose primary cycloadduct cannot readily aromatize, secondary products were obtained and the possibility of generating extended iminium ions could not be explored. Analogous vinyl bromide substrates showed similar reactivity in the cycloaddition step but the primary adducts were found not to survive the reaction conditions, losing molecular $\mathrm{N}_{2}$ and either $\mathrm{Br}^{-}$or $\mathrm{Br}^{*}$, with the formation of alkenyl pyrrolidines (1-azadienes) in most cases. The chemistry of these secondary products, as a group, has not been reported on previously and, as part of a wider study of their diversification, ${ }^{24}$ this work demonstrates that $(4+2)$ and $(2+2)$ annulation modes can be achieved, following $\mathrm{N}$-acylation, via both electrocyclic and free-radical mechanisms. The resulting indolizidine ring systems, and the cyclopenta- and cyclohexa-fused variants, are core components of a variety of alkaloids; there is therefore clear potential to harness the reactivity of 1 -azadienes for the synthesis of specific natural products and their analogues.

\section{Experimental Section}

General information: Except where stated, all reagents were purchased from commercial sources and used without further purification. "Petrol" refers to the fraction of light petroleum ether boiling in the 
range $30-40^{\circ} \mathrm{C}$. "Ether" refers to diethyl ether. MTBE refers to methyl tert-butyl ether. Dry ether, dichloromethane and toluene were obtained from Grubbs canisters under argon. Tetrahydrofuran (THF) was freshly distilled from sodium and benzophenone under a nitrogen atmosphere. Pyridine and triethylamine were distilled prior to use and stored over $\mathrm{KOH}$. All reactions were performed using ovendried glassware under an atmosphere of argon unless otherwise stated. Base-washed glassware was prepared by soaking with saturated aqueous $\mathrm{NaOH}$ solution, washing successively with water and acetone, then oven dried. Reactions were heated, where stated, using oil baths. Pressure tube reactions were carried out in 4 inch "ACE glassware \#15" thick-walled vessels with an approximate volume of $14 \mathrm{~mL}$; tube threads were wrapped with PTFE tape and capped with PTFE screw tops complete with FETFE $^{\circledR}$ O-ring seal (from Sigma-Aldrich). Proton $\left({ }^{1} \mathrm{H}\right)$ and carbon $\left({ }^{13} \mathrm{C}\right)$ NMR spectra were recorded on Bruker AVII-500 (500/125 MHz), Bruker AVIII-400 (400/100 MHz), or Bruker AV-400 (400/100 $\mathrm{MHz}$ ) spectrometers in deuterated solvents; all ${ }^{13} \mathrm{C}$ NMR spectra were proton-decoupled and are reported as such. Chemical shifts $\left(\delta_{\mathrm{H}}\right.$ and $\left.\delta_{\mathrm{C}}\right)$ are quoted in parts per million (ppm), referenced to the appropriate solvent peak. Peak multiplicities are described as singlet (s), doublet (d), triplet (t), quartet (q), quintet (quin), sextet (sext), septet (sept), multiplet (m), apparent (app.) and broad (br). Coupling constants $(J)$ are rounded to the nearest $0.5 \mathrm{~Hz}$. Infrared spectra were recorded using a Bruker Tensor 27 FT-IR spectrometer. Absorption maxima $\left(v_{\max }\right)$ are reported in wavenumbers $\left(\mathrm{cm}^{-1}\right)$ and are described as strong (s), medium (m), weak (w) and broad (br). High-resolution mass spectra (HRMS) were recorded by the staff of the Chemistry Research Laboratory on a Bruker Daltonics MicroTOF spectrometer; mass to charge ratios $(\mathrm{m} / \mathrm{z})$ are reported in Daltons. Melting points were determined using a Griffin MFB-700010U melting point apparatus and are uncorrected. Thin layer chromatography (TLC) was carried out using Merck aluminum-backed DC60 $\mathrm{F}_{254}$ precoated plates $(0.2 \mathrm{~mm})$; spots were visualized by ultraviolet (UV) light $\left(\lambda_{\max }=254 \mathrm{~nm}\right)$ and then stained with anisaldehyde or $\mathrm{KMnO}_{4}$ dips as appropriate. Flash column chromatography was performed using Merck 60 silica gel (particle size 40-63 $\mu \mathrm{m}$ ) and eluted 
with the described solvent(s). The following starting materials were prepared according to literature procedures: 5-(3-chloropropyl)-2,3-dihydrofuran; ${ }^{32}$ 1-(1-ethoxyvinyl)cyclohexan-1-ol; ${ }^{33}$ 5-methylhex4-en-1-ol; ${ }^{34}$ 1-vinylcyclobutanol, ${ }^{35}$ 1-vinylcyclopentanol; ${ }^{36}$ 4-cyclohexylidenebutan-1-ol;37 2-(2-bromo3-methylbut-2-en-1-yl)malonate; ${ }^{38}$ 2,2,5-trimethylhex-4-en-1-ol; ${ }^{39}$ 4-azidobutyroyl chloride. ${ }^{40}$ Reaction schemes for the preparation of substrates, including a key to compound numbers, are included in the Supporting Information.

\section{5-Chloro-2-ethoxypent-1-ene (S1); general procedure for the preparation of w-(chloroalkyl)enol ethers S1-S5}

To an oven-dried $50 \mathrm{~mL}$ round-bottomed flask under an atmosphere of argon was added ethyl vinyl ether $(2.0 \mathrm{~mL}, 20.9 \mathrm{mmol})$ and freshly distilled THF $(15 \mathrm{~mL})$. The flask was cooled to $-78{ }^{\circ} \mathrm{C}$, and tertbutyllithium $(19.0 \mathrm{~mL}, 1.6 \mathrm{M}$ in pentane, $30.4 \mathrm{mmol})$ was added dropwise via syringe over $10 \mathrm{~min}$ causing an intense yellow coloration. The mixture was stirred at $-78^{\circ} \mathrm{C}$ for $1 \mathrm{~h}$ then warmed to $0{ }^{\circ} \mathrm{C}$ and stirred at this temperature for $2 \mathrm{~h}$, during which time the solution changed color from yellow to brown. The mixture was then cooled to $-78^{\circ} \mathrm{C}$, stirred for $1 \mathrm{~h}$, and neat 1-bromo-3-chloropropane $(1.0 \mathrm{~mL}, 10.1$ mmol) was added dropwise, causing a light purple color that faded to colorless within seconds. The reaction mixture was stirred for $14 \mathrm{~h}$ while the cooling bath was allowed to warm from $-78{ }^{\circ} \mathrm{C}$ to $\sim 15$ ${ }^{\circ} \mathrm{C}$. The resulting white slurry was cooled to $0{ }^{\circ} \mathrm{C}$, the reaction was quenched with brine $(6 \mathrm{~mL})$ and the aqueous layer extracted with ether $(3 \times 30 \mathrm{~mL})$. The combined organic extracts were washed successively with water $(5 \mathrm{~mL})$ and brine $(5 \mathrm{~mL})$, then dried $\left(\mathrm{Na}_{2} \mathrm{SO}_{4}\right)$ and concentrated under reduced pressure to yield a colorless oil (1.35 g, 90\%) that was sufficiently pure to be used in the next reaction. An analytical sample was obtained by chromatography using basic alumina and eluting with $1 \%$ triethylamine/petrol. $\mathrm{R}_{f} 0.37$ (petrol); $v_{\max } 2980 \mathrm{w}, 1655 \mathrm{~m}, 1276 \mathrm{~m}, 1077 \mathrm{~s}, 798 \mathrm{~s} ; \delta_{\mathrm{H}}\left(400 \mathrm{MHz}, \mathrm{C}_{6} \mathrm{D}_{6}\right) 1.02(3 \mathrm{H}, \mathrm{t}, J=7.0 \mathrm{~Hz})$, $1.77(2 \mathrm{H}$, app. quin, $J=7.0 \mathrm{~Hz}), 2.13(2 \mathrm{H}, \mathrm{t}, J=7.0 \mathrm{~Hz}), 3.17(2 \mathrm{H}, \mathrm{t}, J=7.0 \mathrm{~Hz}), 3.38(2 \mathrm{H}, \mathrm{q}, J=7.0$ 


\begin{abstract}
$\mathrm{Hz}), 3.79$ and $3.87(2 \times 1 \mathrm{H}, 2 \times \mathrm{d}, J=1.5 \mathrm{~Hz}) ; \delta_{\mathrm{C}}\left(100 \mathrm{MHz}, \mathrm{C}_{6} \mathrm{D}_{6}\right)$ 14.5, 30.6, 32.7, 44.3, 62.7, 81.8, 161.8; HRMS $\left(\mathrm{FI}^{+}\right) \mathrm{m} / z$ : $\left[\mathrm{M}^{+}\right]$Calcd for $\mathrm{C}_{7} \mathrm{H}_{13}{ }^{35} \mathrm{ClO}$ 148.0649; Found 148.0658.
\end{abstract}

\title{
6-Chloro-2-ethoxyhex-1-ene (S2)
}

Obtained as a colorless oil (1.36 g, quantitative) from ethyl vinyl ether and 1-chloro-4-iodobutane. $\mathrm{R}_{f}$ 0.25 (petrol); $v_{\max } 2934 \mathrm{w}, 2972 \mathrm{w}, 1653 \mathrm{~m}, 1277 \mathrm{~m}, 1081 \mathrm{~m}, 796 \mathrm{~s}, 652 \mathrm{~m} ; \delta_{\mathrm{H}}\left(400 \mathrm{MHz}, \mathrm{C}_{6} \mathrm{D}_{6}\right) 1.06(3 \mathrm{H}$, $\mathrm{t}, J=7.0 \mathrm{~Hz}), 1.42-1.55(4 \mathrm{H}, \mathrm{m}), 1.99(2 \mathrm{H}, \mathrm{t}, J=7.0 \mathrm{~Hz}), 3.08(2 \mathrm{H}, \mathrm{t}, J=6.5 \mathrm{~Hz}), 3.44(2 \mathrm{H}, \mathrm{q}, J=$ $7.0 \mathrm{~Hz}), 3.82$ and $3.86(2 \times 1 \mathrm{H}, 2 \times \mathrm{d}, J=1.5 \mathrm{~Hz}) ; \delta_{\mathrm{C}}\left(100 \mathrm{MHz}, \mathrm{C}_{6} \mathrm{D}_{6}\right)$ 14.5, 24.9, 32.2, 34.7, 44.7, 62.7, 81.2, 163.0; HRMS (FI') m/z: [M+] Calcd for $\mathrm{C}_{8} \mathrm{H}_{15}{ }^{35} \mathrm{ClO}$ 162.0806; Found 162.0813.

\section{5-(3-Chloropropyl)-2,3-dihydrofuran $(\mathrm{S3})^{32}$}

Obtained as a pale yellow oil (1.07 g, quantitative) from dihydrofuran and 1-bromo-3-chloropropane. $\mathrm{R}_{f}$ 0.60 (ethyl acetate/petrol, 1:10); $v_{\max } 2958 \mathrm{w}, 2862 \mathrm{w}, 1668 \mathrm{~m}, 1003 \mathrm{~s}, 930 \mathrm{~s}, 724 \mathrm{~s}, 651 \mathrm{~s} ; \delta_{\mathrm{H}}(400 \mathrm{MHz}$, $\left.\mathrm{C}_{6} \mathrm{D}_{6}\right) 1.71(2 \mathrm{H}$, app. quin, $J=7.0 \mathrm{~Hz}), 2.10(2 \mathrm{H}$, tq, $J=7.0,1.0 \mathrm{~Hz}), 2.24(2 \mathrm{H}, \mathrm{tq}, J=9.0,2.0 \mathrm{~Hz})$, $3.13(2 \mathrm{H}, \mathrm{t}, J=6.0 \mathrm{~Hz}), 3.99(2 \mathrm{H}, \mathrm{t}, J=9.0 \mathrm{~Hz}), 4.40(1 \mathrm{H}$, sept, $J=1.0 \mathrm{~Hz}) ; \delta_{\mathrm{C}}\left(100 \mathrm{MHz}, \mathrm{C}_{6} \mathrm{D}_{6}\right) 25.4$, 30.0, 30.2, 44.3, 69.8, 94.6, 157.7; $\mathrm{HRMS}\left(\mathrm{FI}^{+}\right) \mathrm{m} / z$ : $\left[\mathrm{M}^{+}\right]$Calcd for $\mathrm{C}_{7} \mathrm{H}_{11}{ }^{35} \mathrm{ClO} 146.0493$; Found 146.0502 .

\section{5-(4-Chlorobutyl)-2,3-dihydrofuran (S4)}

Obtained as a pale yellow oil (824 mg, quantitative) from dihydrofuran and 1-chloro-4-iodobutane. $\mathrm{R}_{f}$ 0.20 (petrol); $v_{\max } 2935 \mathrm{~s}, 2861 \mathrm{~m}, 1667 \mathrm{~s}, 1005 \mathrm{~s}, 725 \mathrm{~s} ; \delta_{\mathrm{H}}\left(400 \mathrm{MHz}, \mathrm{C}_{6} \mathrm{D}_{6}\right) 1.41-1.49(4 \mathrm{H}, \mathrm{m}), 1.93-$ $1.97(2 \mathrm{H}, \mathrm{m}), 2.28(2 \mathrm{H}, \mathrm{tq}, J=9.5,2.0 \mathrm{~Hz}), 3.06(2 \mathrm{H}, \mathrm{t}, J=6.0 \mathrm{~Hz}), 4.05(2 \mathrm{H}, \mathrm{t}, J=9.5 \mathrm{~Hz}), 4.42(1$ H, app. sept, $J=1.0 \mathrm{~Hz}) ; \delta_{\mathrm{C}}\left(100 \mathrm{MHz}, \mathrm{C}_{6} \mathrm{D}_{6}\right) 24.3,27.4,30.3,32.3,44.6,69.8,94.0,158.8$; HRMS $\left(\mathrm{ESI}^{+}\right) \mathrm{m} / z:[\mathrm{M}+\mathrm{H}]^{+}$Calcd for $\mathrm{C}_{8} \mathrm{H}_{14}{ }^{35} \mathrm{ClO}$ 161.0728; Found 161.0725. 


\section{6-(4-Chlorobutyl)-3,4-dihydro-2H-pyran (S5)}

Obtained as a pale yellow oil (1.08 g, quantitative) from dihydropyran and 1-chloro-4-iodobutane. $\mathrm{R}_{f}$ 0.80 (ethyl acetate/petrol, 1:1); $v_{\max } 2932 \mathrm{~m}, 2868 \mathrm{w}, 1675 \mathrm{~m}, 1234 \mathrm{~m}, 1062 \mathrm{~s}, 762 \mathrm{~s}, 649 \mathrm{~m} ; \delta_{\mathrm{H}}(400 \mathrm{MHz}$, $\left.\mathrm{C}_{6} \mathrm{D}_{6}\right) 1.40-1.51(6 \mathrm{H}, \mathrm{m}), 1.75-1.80(2 \mathrm{H}, \mathrm{m}), 1.90-1.92(2 \mathrm{H}, \mathrm{m}), 3.08-3.12(2 \mathrm{H}, \mathrm{m}), 3.72(2 \mathrm{H}, \mathrm{t}, J=$ $5.0 \mathrm{~Hz}), 4.37(1 \mathrm{H}, \mathrm{t}, J=4.0 \mathrm{~Hz}) ; \delta_{\mathrm{C}}\left(100 \mathrm{MHz}, \mathrm{C}_{6} \mathrm{D}_{6}\right) 20.6,22.7,24.6,32.3,33.9,44.8,66.0,95.6$, 154.3; HRMS $\left(\mathrm{FI}^{+}\right) \mathrm{m} / z$ : $\left[\mathrm{M}^{+}\right]$Calcd for $\mathrm{C}_{9} \mathrm{H}_{15}{ }^{35} \mathrm{ClO}$ 174.0806; Found 174.0804.

\section{5-Azido-2-ethoxypent-1-ene (11a); general procedure for the preparation of azides 11a,b and 12a-c}

To an oven-dried $50 \mathrm{~mL}$ round-bottomed flask was added chloride $\mathbf{S 1}(211 \mathrm{mg}, 1.42 \mathrm{mmol}), \mathrm{NaN}_{3}(146$ $\mathrm{mg}, 2.25 \mathrm{mmol})$ and DMSO $(2.0 \mathrm{~mL})$. The slurry was stirred under an atmosphere of argon at $50{ }^{\circ} \mathrm{C}$ for $21 \mathrm{~h}$ then cooled to RT; ether $(50 \mathrm{~mL})$ was added and the solution was washed successively with water $(10 \times 1 \mathrm{~mL})$ and brine $(1 \mathrm{~mL})$. The organic layer was then dried $\left(\mathrm{Na}_{2} \mathrm{SO}_{4}\right)$ and concentrated under reduced pressure to afford a yellow oil (160 mg, 73\%) that was sufficiently pure to be used in the next reaction. $\mathrm{R}_{f} 0.37$ (petrol); $v_{\max } 2932 \mathrm{w}, 2092 \mathrm{~s}, 1654 \mathrm{~m}, 1271 \mathrm{~m}, 1086 \mathrm{~m}, 799 \mathrm{~m} ; \delta_{\mathrm{H}}\left(400 \mathrm{MHz}, \mathrm{C}_{6} \mathrm{D}_{6}\right) 1.03(3 \mathrm{H}, \mathrm{t}, J$ $=7.0 \mathrm{~Hz}), 1.53(2 \mathrm{H}$, app. quin, $J=7.0 \mathrm{~Hz}), 2.00(2 \mathrm{H}, \mathrm{t}, J=7.0 \mathrm{~Hz}), 2.74(2 \mathrm{H}, \mathrm{t}, J=7.0 \mathrm{~Hz}), 3.39(2$ $\mathrm{H}, \mathrm{q}, J=7.0 \mathrm{~Hz}), 3.79$ and $3.84(2 \times 1 \mathrm{H}, 2 \times \mathrm{d}, J=1.5 \mathrm{~Hz}) ; \delta_{\mathrm{C}}\left(100 \mathrm{MHz}, \mathrm{C}_{6} \mathrm{D}_{6}\right) 14.5,26.9,32.5,50.6$, 62.8, 81.6, 162.1; HRMS (ESI $\left.{ }^{+}\right) m / z:[\mathrm{M}+\mathrm{H}]^{+}$Calcd for $\mathrm{C}_{7} \mathrm{H}_{14} \mathrm{~N}_{3} \mathrm{O}$ 156.1131; Found 156.0933 .

\section{6-Azido-2-ethoxyhex-1-ene (11b)}

Obtained as a pale yellow oil (1.02 g, 84\%) from chloride $\mathbf{S 2}$. $\mathrm{R}_{f} 0.25$ (petrol); $v_{\max } 2980 \mathrm{w}, 2091 \mathrm{~s}, 1653 \mathrm{~m}$, 1094m, 796s; $\delta_{\mathrm{H}}\left(400 \mathrm{MHz}, \mathrm{C}_{6} \mathrm{D}_{6}\right) 1.07(3 \mathrm{H}, \mathrm{t}, J=7.0 \mathrm{~Hz}), 1.22(2 \mathrm{H}$, app. quin, $J=7.0 \mathrm{~Hz}), 1.39(2 \mathrm{H}$, app. quin, $J=7.5 \mathrm{~Hz}) 1.98(2 \mathrm{H}, \mathrm{t}, J=7.5 \mathrm{~Hz}), 2.65(2 \mathrm{H}, \mathrm{t}, J=7.0 \mathrm{~Hz}), 3.45(2 \mathrm{H}, \mathrm{q}, J=7.0 \mathrm{~Hz}), 3.83$ 
and $3.87(2 \times 1 \mathrm{H}, 2 \times \mathrm{d}, J=1.0 \mathrm{~Hz}) ; \delta_{\mathrm{C}}\left(100 \mathrm{MHz}, \mathrm{C}_{6} \mathrm{D}_{6}\right) 14.5,24.7,28.4,34.9,51.1,62.7,81.2,163.0$; HRMS (ESI $\left.{ }^{+}\right) m / z:[\mathrm{M}+\mathrm{H}]^{+}$Calcd for $\mathrm{C}_{8} \mathrm{H}_{16} \mathrm{~N}_{3} \mathrm{O}$ 170.1288; Found 170.1289 .

\section{5-(3-Azidopropyl)-2,3-dihydrofuran (12a)}

Obtained as a yellow oil (178 mg, 55\%) from chloride S3. $\mathrm{R}_{f} 0.60$ (ethyl acetate/petrol, 1:10); $v_{\max }$ 2933w, 2864w, 2095s, 1668w, 1258m, 1005m, 961m. $\delta_{\mathrm{H}}\left(400 \mathrm{MHz}, \mathrm{C}_{6} \mathrm{D}_{6}\right) 1.47$ (2 H, app. quin, $J=7.0$ Hz), $1.97(2 \mathrm{H}, \mathrm{tq}, J=7.0,1.0 \mathrm{~Hz}), 2.25(2 \mathrm{H}, \mathrm{tq}, J=9.5,2.0 \mathrm{~Hz}), 2.72(2 \mathrm{H}, \mathrm{t}, J=7.0 \mathrm{~Hz}), 4.01(2 \mathrm{H}, \mathrm{t}$, $J=9.5 \mathrm{~Hz}), 4.39(1 \mathrm{H}$, sept, $J=1.0 \mathrm{~Hz}) ; \delta_{\mathrm{C}}\left(100 \mathrm{MHz}, \mathrm{C}_{6} \mathrm{D}_{6}\right) 25.2,26.3,30.3,50.7,69.8,94.5,157.9$; HRMS (FI') m/z: [M+] Calcd for $\mathrm{C}_{7} \mathrm{H}_{11} \mathrm{~N}_{3} \mathrm{O}$ 153.0897; Found 153.0899.

\section{5-(4-Azidobutyl)-2,3-dihydrofuran (12b)}

Obtained as a pale yellow oil (497 mg, 84\%) from chloride $\mathbf{S} 4 . \mathbf{R}_{f}=0.20$ (petrol); $v_{\max } 2941 \mathrm{~m}, 2864 \mathrm{~m}$, 2091s, 1667m, 1005m, 723m; $\delta_{\mathrm{H}}\left(400 \mathrm{MHz}, \mathrm{C}_{6} \mathrm{D}_{6}\right) 1.17-1.24(2 \mathrm{H}, \mathrm{m}), 1.30-1.38(2 \mathrm{H}, \mathrm{m}), 1.94(2 \mathrm{H}$, tq, $J=7.5,1.0 \mathrm{~Hz}), 2.29(2 \mathrm{H}, \mathrm{tq}, J=9.5,2.0 \mathrm{~Hz}), 2.63(2 \mathrm{H}, \mathrm{t}, J=7.0 \mathrm{~Hz}), 4.06(2 \mathrm{H}, \mathrm{t}, J=9.5 \mathrm{~Hz})$, $4.43(1 \mathrm{H}$, app. sept, $J=1.0 \mathrm{~Hz}) ; \delta_{\mathrm{C}}\left(100 \mathrm{MHz}, \mathrm{C}_{6} \mathrm{D}_{6}\right) 24.0,27.7,28.5,30.3,51.1,69.8,94.0,158.8$; HRMS $\left(\mathrm{ESI}^{+}\right) \mathrm{m} / z:[\mathrm{M}+\mathrm{H}]^{+}$Calcd for $\mathrm{C}_{8} \mathrm{H}_{14} \mathrm{~N}_{3} \mathrm{O}$ 168.1131; Found 168.1131 .

\section{6-(4-Azidobutyl)-3,4-dihydro-2H-pyran (12c)}

Obtained as a pale yellow oil (398 $\mathrm{mg}, 72 \%$ ) from chloride $\mathbf{S 5}$. $\mathrm{R}_{f} 0.80$ (ethyl acetate/petrol, 1:1); $v_{\max }$ 2970m, 2867w, 2090s, 1739m, 1675m, 1233m, 1063m, 763m; $\delta_{\mathrm{H}}\left(400 \mathrm{MHz}, \mathrm{C}_{6} \mathrm{D}_{6}\right) 1.25(2 \mathrm{H}$, app. quin, $J=7.0 \mathrm{~Hz}), 1.36-1.49(4 \mathrm{H}, \mathrm{m}), 1.78-1.81(2 \mathrm{H}, \mathrm{m}), 1.92(2 \mathrm{H}, \mathrm{t}, J=7.5 \mathrm{~Hz}), 2.68(2 \mathrm{H}, \mathrm{t}, J=7.0 \mathrm{~Hz})$, $3.74(2 \mathrm{H}, \mathrm{t}, J=5.0 \mathrm{~Hz}), 4.39(1 \mathrm{H}, \mathrm{t}, J=4.0 \mathrm{~Hz}) ; \delta_{\mathrm{C}}\left(100 \mathrm{MHz}, \mathrm{C}_{6} \mathrm{D}_{6}\right) 20.6,22.8,24.3,28.4,34.2,51.2$, 66.0, 95.6, 154.3; HRMS $\left(\mathrm{FI}^{+}\right) \mathrm{m} / z$ : $\left[\mathrm{M}^{+}\right]$Calcd for $\mathrm{C}_{9} \mathrm{H}_{15} \mathrm{~N}_{3} \mathrm{O}$ 181.1210; Found 181.1210. 


\section{3a-Ethoxy-3a,4,5,6-tetrahydro-3H-pyrrolo[1,2-c][1,2,3]triazole (14a); general procedure for thermolysis of azides 11 and 12}

To an oven-dried $14 \mathrm{~mL}$ Ace pressure tube was added azide 11a (467 mg, $3.01 \mathrm{mmol})$, dry toluene (8.0 $\mathrm{mL}$ ), and solid $\mathrm{K}_{2} \mathrm{CO}_{3}(128 \mathrm{mg}, 0.926 \mathrm{mmol})$. The vessel was flushed with argon, sealed with a PTFE screw cap with $\mathrm{FETFE}^{\circledR}$ O-ring and stirred at $130{ }^{\circ} \mathrm{C}$ for $96 \mathrm{~h}$, during which time the white slurry became brown. The tube was cooled to RT and the solution was filtered then concentrated to yield a yellow oil (460 mg), revealed by ${ }^{1} \mathrm{H}-\mathrm{NMR}$ spectroscopy to be a 4:1 mixture of the title compound (14a) and 5,6dihydro-4H-pyrrolo[1,2-c] $[1,2,3]$ triazole (16). ${ }^{9}$ An analytical sample of 14a was obtained as a colorless oil by chromatography over basic alumina eluting with $1 \%$ triethylamine in $5 \%$ ether/petrol. $\mathrm{R}_{f}=0.28$ (ether/petrol, 1:1); $v_{\max } 2971 \mathrm{~m}, 1476 \mathrm{w}, 1443 \mathrm{w}, 1366 \mathrm{~m}, 1216 \mathrm{~s}, 1106 \mathrm{~s} ; \delta_{\mathrm{H}}\left(400 \mathrm{MHz}, \mathrm{C}_{6} \mathrm{D}_{6}\right) 0.98(3 \mathrm{H}, \mathrm{t}$, $J=7.0 \mathrm{~Hz}), 1.03-1.18(2 \mathrm{H}, \mathrm{m}), 1.43-1.53(1 \mathrm{H}, \mathrm{m}), 1.76(1 \mathrm{H}, \mathrm{dt}, J=12.5,6.5 \mathrm{~Hz}), 2.90-2.98(1 \mathrm{H}, \mathrm{m}$, $J=7.0 \mathrm{~Hz}), 3.12-3.23(2 \mathrm{H}, \mathrm{m}), 3.45(1 \mathrm{H}, \mathrm{dt}, J=11.5,6.0 \mathrm{~Hz}), 3.60$ and $4.10(2 \times 1 \mathrm{H}, 2 \times \mathrm{d}, J=18.5$ $\mathrm{Hz}) ; \delta_{\mathrm{C}}\left(100 \mathrm{MHz}, \mathrm{C}_{6} \mathrm{D}_{6}\right) 15.5,24.9,36.9,49.2,57.9,72.6,101.0 ; \mathrm{HRMS}\left(\mathrm{ESI}^{+}\right) \mathrm{m} / z:[\mathrm{M}+\mathrm{H}]^{+}$Calcd for $\mathrm{C}_{7} \mathrm{H}_{14} \mathrm{~N}_{3} \mathrm{O}$ 156.1131; Found 156.1138. Data for 16: $\mathrm{R}_{f}=0.10$ (ethyl acetate/petrol, 1:4); $v_{\max } 2961 \mathrm{~m}$, $1654 \mathrm{w}, 1454 \mathrm{w}, 1438 \mathrm{~m}, 1311 \mathrm{~m}, 1224 \mathrm{~m}, 1177 \mathrm{~m}, 1094 \mathrm{~s}, 724 \mathrm{~m} ; \delta_{\mathrm{H}}\left(400 \mathrm{MHz}, \mathrm{CDCl}_{3}\right) 2.80(2 \mathrm{H}$, app. quin, $J=7.0 \mathrm{~Hz}), 2.92(2 \mathrm{H}, \mathrm{t}, J=7.0 \mathrm{~Hz}), 4.33(2 \mathrm{H}, \mathrm{t}, J=7.0 \mathrm{~Hz}), 7.39(1 \mathrm{H}, \mathrm{s}) ; \delta_{\mathrm{C}}\left(100 \mathrm{MHz}, \mathrm{CDCl}_{3}\right)$ 20.7, 28.4, 46.3, 126.9, 142.0; HRMS $\left(\mathrm{ESI}^{+}\right) \mathrm{m} / z:[\mathrm{M}+\mathrm{H}]^{+}$Calcd for $\mathrm{C}_{5} \mathrm{H}_{8} \mathrm{~N}_{3}$ 110.0713; Found 110.0710.

\section{3a-Ethoxy-3,3a,4,5,6,7-hexahydro[1,2,3]triazolo[1,5-a]pyridine (14b)}

The crude product (69 mg), a yellow oil, was obtained from azide $\mathbf{1 1 b}(70 \mathrm{mg}, 0.43 \mathrm{mmol})$ and shown by ${ }^{1} \mathrm{H}$ NMR analysis to be a $6: 4$ mixture of the title compound (14b) and 4,5,6,7tetrahydro[1,2,3]triazolo[1,5-a]pyridine (17). ${ }^{9,10}$ An analytical sample of $\mathbf{1 4 b}$ was obtained as a colorless oil by chromatography over basic alumina eluting with $1 \%$ triethylamine in $5 \%$ ether/petrol. $\mathrm{R}_{f} 0.33$ (petrol/ether, 1:1); $v_{\max } 2971 \mathrm{w}, 2943 \mathrm{w}, 1444 \mathrm{w}, 1316 \mathrm{~m}, 1217 \mathrm{~m}, 1046 \mathrm{~m}, 904 \mathrm{w} ; \delta_{\mathrm{H}}\left(400 \mathrm{MHz}, \mathrm{C}_{6} \mathrm{D}_{6}\right)$ 
0.90-0.98 (1 H, m) overlays $0.94(3 \mathrm{H}, \mathrm{t}, J=7.0 \mathrm{~Hz}), 1.10-1.27(3 \mathrm{H}, \mathrm{m}), 1.66(1 \mathrm{H}, \mathrm{qt}, J=13.0,4.0$ $\mathrm{Hz}), 1.91(1 \mathrm{H}, \mathrm{dt}, J=13.0,3.0 \mathrm{~Hz}), 2.71$ and $2.79(2 \times 1 \mathrm{H}, 2 \times \mathrm{dq}, J=9.0,7.0 \mathrm{~Hz}), 3.05(1 \mathrm{H}, \mathrm{td}, J=$ 12.0, 4.0 Hz), $3.34(1 \mathrm{H}, \mathrm{d}, J=17.5 \mathrm{~Hz}), 3.90-3.94(1 \mathrm{H}, \mathrm{m}), 3.99(1 \mathrm{H}, \mathrm{d}, J=17.5 \mathrm{~Hz}) ; \delta_{\mathrm{C}}(100 \mathrm{MHz}$, $\left.\mathrm{C}_{6} \mathrm{D}_{6}\right)$ 15.2, 20.7, 25.0, 34.7, 43.0, 58.1, 71.4, 88.0; HRMS $\left(\mathrm{ESI}^{+}\right) \mathrm{m} / z$ : $[\mathrm{M}+\mathrm{H}]^{+}$Calcd for $\mathrm{C}_{8} \mathrm{H}_{16} \mathrm{~N}_{3} \mathrm{O}$ 170.1288; Found 170.1283. Data for 17: $\mathrm{R}_{f} 0.10$ (petrol/ethyl acetate, 4:1); $v_{\max } 2950 \mathrm{~m}, 2868 \mathrm{w}, 2095 \mathrm{w}$, $1552 \mathrm{w}, 1431 \mathrm{~m}, 1236 \mathrm{~s}, 990 \mathrm{~s}, 811 \mathrm{~m}, 645 \mathrm{~m} ; \delta_{\mathrm{H}}\left(400 \mathrm{MHz}, \mathrm{CDCl}_{3}\right) 1.88-1.94(2 \mathrm{H}, \mathrm{m}), 2.05-2.10(2 \mathrm{H}$, m), $2.84(2 \mathrm{H}, \mathrm{t}, J=6.5 \mathrm{~Hz}), 4.37(2 \mathrm{H}, \mathrm{t}, J=6.0 \mathrm{~Hz}) 7.44(1 \mathrm{H}, \mathrm{s}) ; \delta_{\mathrm{C}}\left(100 \mathrm{MHz}, \mathrm{CDCl}_{3}\right) 20.3$ (two peaks), 22.9, 46.1, 130.9, 133.3 .

\section{2,3,3a,7,8,9-Hexahydrofuro[2,3-d]pyrrolo[1,2-c][1,2,3]triazole (15a)}

The crude product (38 mg), a yellow oil, was obtained from azide 12a (40 mg, $0.26 \mathrm{mmol}$ ). An analytical sample of 15a was obtained as a colorless oil by chromatography over basic alumina eluting with $1 \%$ triethylamine in 5\% ether/petrol. $\mathrm{R}_{f} 0.20$ (petrol/ether, $1: 1$ ); $v_{\max } 2951 \mathrm{w}, 2872 \mathrm{w}, 1491 \mathrm{~m}, 1442 \mathrm{~m}, 1121 \mathrm{~s}$, $1029 \mathrm{~s}, 991 \mathrm{~s}, 843 \mathrm{~m}, 676 \mathrm{~m} ; \delta_{\mathrm{H}}\left(400 \mathrm{MHz}, \mathrm{C}_{6} \mathrm{D}_{6}\right) 1.08-1.16(1 \mathrm{H}, \mathrm{m}), 1.33-1.46(2 \mathrm{H}, \mathrm{m}) .1 .62-1.73(1$ H, m), 1.80-1.90 (2 H, m), 3.12-3.23 (2 H, m), 3.44-3.53 (2 H, m), $4.17(1 \mathrm{H}, \mathrm{d}, J=9.5 \mathrm{~Hz}) ; \delta_{\mathrm{C}}(100$ $\left.\mathrm{MHz}, \mathrm{C}_{6} \mathrm{D}_{6}\right)$ 25.5, 32.0, 33.5, 48.9, 65.2, 85.6, 108.0; HRMS $\left(\mathrm{ESI}^{+}\right) \mathrm{m} / z$ : $\left[\mathrm{M}+\mathrm{H}-\mathrm{N}_{2}\right]^{+}$Calcd for $\mathrm{C}_{7} \mathrm{H}_{12} \mathrm{NO}$ 126.0913; Found 126.0918.

\section{3,3a,7,8,9,10-Hexahydro-2H-furo[3', 3 ':4,5]triazolo[1,5-a]pyridine (15b)}

The crude product (42 mg), a yellow oil, was obtained from azide 12b (40 mg, $0.26 \mathrm{mmol}$ ). An analytical sample of $\mathbf{1 5 b}$ was obtained as a colorless oil by chromatography over basic alumina eluting with $1 \%$ triethylamine in 5\% ether/petrol. $\mathrm{R}_{f}=0.19$ (petrol ether, 1:1) $v_{\max } 2946 \mathrm{~m}, 2864 \mathrm{w}, 1440 \mathrm{~m}, 1366 \mathrm{~s}, 1216 \mathrm{~s}$, $1032 \mathrm{~m} ; \delta_{\mathrm{H}}\left(400 \mathrm{MHz}, \mathrm{C}_{6} \mathrm{D}_{6}\right) 1.10-1.21(3 \mathrm{H}, \mathrm{m}), 1.32(1 \mathrm{H}$, dquin, $J=13.0,3.5 \mathrm{~Hz}), 1.60-1.75(3 \mathrm{H}$, m), $2.04(1 \mathrm{H}, \mathrm{dd}, J=12.0,5.0 \mathrm{~Hz}), 3.14(1 \mathrm{H}, \mathrm{ddd}, J=12.0,8.5,5.0 \mathrm{~Hz}), 3.40(1 \mathrm{H}, \mathrm{td}, J=12.0,4.5$ 


\begin{abstract}
$\mathrm{Hz}), 3.51(1 \mathrm{H}, \mathrm{ddd}, J=8.0,7.5,1.0 \mathrm{~Hz}), 3.94-3.98(1 \mathrm{H}, \mathrm{m}), 4.09(1 \mathrm{H}, \mathrm{d}, J=8.0 \mathrm{~Hz}) ; \delta_{\mathrm{C}}(100 \mathrm{MHz}$, $\left.\mathrm{C}_{6} \mathrm{D}_{6}\right)$ 21.1, 25.3, 32.1, 32.9, 43.4, 65.8, 83.7, 95.1; HRMS $\left(\mathrm{ESI}^{+}\right) \mathrm{m} / z$ : $[\mathrm{M}+\mathrm{H}]^{+}$Calcd for $\mathrm{C}_{8} \mathrm{H}_{14} \mathrm{~N}_{3} \mathrm{O}$ 168.1131; Found 168.1136.
\end{abstract}

\title{
12-Oxa-6,7,8-triazatricyclo[7.4.0.0 $\left.{ }^{1,6}\right]$ tridec-7-ene (15c)
}

The crude product (114 mg), a yellow waxy solid, was obtained from azide 12c (147 mg, $0.81 \mathrm{mmol})$. The title compound (15c) was obtained pure, as a colorless oil, by chromatography over basic alumina, eluting with $1 \%$ triethylamine in $10 \%$ ether/petrol $(30 \mathrm{mg}, 20 \%) . \mathrm{R}_{f} 0.30$ (petrol/ether, $\left.1: 1\right) ; v_{\max } 2942 \mathrm{~s}$, 2859m, 1445m, 1094m, 1033s, 942m, 808m; $\delta_{\mathrm{H}}\left(400 \mathrm{MHz}, \mathrm{C}_{6} \mathrm{D}_{6}\right)$ 0.93-1.03 (2 H, m), 1.23-1.30 (3 H, m), 1.39-1.51 (2 H, m), 1.57-1.69 (1 H, m), $1.73(1 \mathrm{H}, \mathrm{dt}, J=13.0,3.0 \mathrm{~Hz}), 2.13-2.20(1 \mathrm{H}, \mathrm{m}), 2.95-$ $3.01(1 \mathrm{H}, \mathrm{m}), 3.24-3.31(2 \mathrm{H}, \mathrm{m}), 3.39-3.46$ (1 H, m), 3.89-3.94 (1 H, m); $\delta_{\mathrm{C}}\left(100 \mathrm{MHz}, \mathrm{C}_{6} \mathrm{D}_{6}\right) 20.1$, 21.2, 21.6, 24.9, 30.4, 43.0. 60.3, 76.5, 87.7; HRMS $\left(\mathrm{ESI}^{+}\right) \mathrm{m} / z:[\mathrm{M}+\mathrm{H}]^{+}$Calcd for $\mathrm{C}_{9} \mathrm{H}_{16} \mathrm{~N}_{3} \mathrm{O}$ 182.1288; Found 182.1285.

\section{2-(4,5,6,7-Tetrahydro[1,2,3]triazolo[1,5-a]pyridin-3-yl)ethanol (18)}

The title compound was obtained from a failed attempt to achieve iminium formation and allylation from cycloadduct $\mathbf{1 5 b}$. To an oven-dried $10 \mathrm{~mL}$ round-bottomed flask was added cycloadduct $\mathbf{1 5 b}$ (19 $\mathrm{mg}$, $0.11 \mathrm{mmol})$ and dry dichloromethane $(2.0 \mathrm{~mL})$; the flask was flushed with argon and the mixture cooled to $-78^{\circ} \mathrm{C}$. Allyltributyl tin $(38 \mu \mathrm{L}, 0.12 \mathrm{mmol})$ was added via syringe, followed by TMSOTf $(28 \mu \mathrm{L}$, $0.15 \mathrm{mmol}$ ) after $10 \mathrm{~min}$. The reaction mixture was stirred for $16 \mathrm{~h}$ during which time the cooling bath was allowed to warm from $-78^{\circ} \mathrm{C}$ to $\sim 12^{\circ} \mathrm{C}$; the reaction was quenched with saturated aqueous $\mathrm{NaHCO}_{3}$ solution $(1.0 \mathrm{~mL})$, and extracted with dichloromethane $(3 \times 7 \mathrm{~mL})$. The combined extracts were dried $\left(\mathrm{K}_{2} \mathrm{CO}_{3}\right)$ and concentrated to yield a colorless oil with solid particles suspended within (62 mg). The solid was found to be insoluble in $\mathrm{C}_{6} \mathrm{D}_{6}$ but dissolved in $\mathrm{CDCl}_{3}$, and was revealed to be the triazole 18 . 
The crude triazole was passed through a silica gel column gradient eluting with ethyl acetate to methanol, yielding a the title compound as a colorless oil $(7.0 \mathrm{mg}, 38 \%)$. $\mathrm{R}_{f} 0.18$ (petrol/ethyl acetate, $\left.1: 1\right) ; v_{\max }$ $3362 \mathrm{br}, 2949 \mathrm{~s}, 2867 \mathrm{~m}, 1574 \mathrm{~m}, 1224 \mathrm{~m}, 1052 \mathrm{~s}, 668 \mathrm{~m}$; $\delta_{\mathrm{H}}\left(400 \mathrm{MHz}, \mathrm{CDCl}_{3}\right) 1.89-1.95$ (2 H, m), $2.03-$ $2.09(2 \mathrm{H}, \mathrm{m}), 2.75(2 \mathrm{H}, \mathrm{t}, J=6.5 \mathrm{~Hz}), 2.83(2 \mathrm{H}, \mathrm{t}, J=6.0 \mathrm{~Hz}), 3.97(2 \mathrm{H}, \mathrm{t}, J=6.0 \mathrm{~Hz}), 4.35(2 \mathrm{H}, \mathrm{t}, J$ $=6.0 \mathrm{~Hz}) ; \delta_{\mathrm{C}}\left(100 \mathrm{MHz}, \mathrm{CDCl}_{3}\right)$ 19.9, 20.2, 22.8, 27.8, 46.4, 61.7 (the $4^{\circ}$-carbons were not observed in this weak NMR sample); HRMS (ESI $\left.{ }^{+}\right) m / z$ : $[\mathrm{M}+\mathrm{H}]^{+}$Calcd for $\mathrm{C}_{8} \mathrm{H}_{14} \mathrm{~N}_{3} \mathrm{O}$ 168.1131; Found 168.1130.

\section{Ethyl 4-cyclohexylidene-4-ethoxybutanoate (S6)}

To an oven-dried $25 \mathrm{~mL}$ round-bottomed flask equipped with stirrer bar, distillation head and thermometer was added triethylorthoacetate $(4.0 \mathrm{~mL}), 1$-(1-ethoxyvinyl)cyclohexan-1-ol ${ }^{33}(340 \mathrm{mg}, 2.0$ mmol), and a few crystals of hydroquinone. The set-up was flushed with argon, then placed into a preheated oil bath at $135^{\circ} \mathrm{C}$. Propionic acid ( 2 drops) was added at hourly intervals over $5 \mathrm{~h}$, then the reaction mixture was heated for a further $16 \mathrm{~h}$. Ethanol was observed distilling off at $78^{\circ} \mathrm{C}$. The mixture was cooled to RT, diluted with ether $(70 \mathrm{~mL})$ and washed successively with saturated aqueous $\mathrm{NH}_{4} \mathrm{Cl}$ $(20 \mathrm{~mL})$, saturated aqueous $\mathrm{NaHCO}_{3}(20 \mathrm{~mL})$, and brine $(20 \mathrm{~mL})$. The organic layer was dried $\left(\mathrm{Na}_{2} \mathrm{SO}_{4}\right)$ and concentrated onto basic alumina for purification over alumina eluting with $2 \%$ triethylamine in $5 \%$ ether/petrol (30 mg, 6\%). $\mathrm{R}_{f} 0.66$ (petrol/ether, 2:1); $v_{\max } 2926 \mathrm{~m}, 1735 \mathrm{~s}, 1164 \mathrm{~m}, 1112 \mathrm{~m}, 1046 \mathrm{~m} ; \delta_{\mathrm{H}}(400$ MHz, $\left.\mathrm{C}_{6} \mathrm{D}_{6}\right) 0.96(3 \mathrm{H}, \mathrm{t}, J 7.5 \mathrm{~Hz}), 1.06(3 \mathrm{H}, \mathrm{t}, J=7.5 \mathrm{~Hz}), 1.41-1.49(6 \mathrm{H}, \mathrm{m}), 2.00-2.07(2 \mathrm{H}, \mathrm{m})$, 2.29-2.34 (2 H, m), 2.42-2.48 (2 H, m), 2.52-2.57 (2 H, m), $3.42(2 \mathrm{H}, \mathrm{q}, J=7.5 \mathrm{~Hz}), 3.97(2 \mathrm{H}, \mathrm{q}, J=$ $7.5 \mathrm{~Hz}) ; \delta_{\mathrm{C}}\left(100 \mathrm{MHz}, \mathrm{C}_{6} \mathrm{D}_{6}\right)$ 14.3, 15.6, 22.9, 27.2, 27.7, 28.1, 28.5, 29.7, 32.9, 60.1, 64.9, 123.7, 145.1, 172.8; HRMS $\left(\mathrm{ESI}^{+}\right) \mathrm{m} / z$ : $[\mathrm{M}+\mathrm{Na}]^{+} \mathrm{Calcd}$ for $\mathrm{C}_{14} \mathrm{H}_{24} \mathrm{NaO}_{3}$ 263.1618; Found 263.1621. ${ }^{41}$

(4-Azido-1-ethoxybutylidene)cyclohexane (19) 
To an oven-dried $25 \mathrm{~mL}$ round-bottomed flask was added ester S6 (30 mg, $0.12 \mathrm{mmol}$ ) and THF (1.0 $\mathrm{mL})$, and the solution was stirred at $0^{\circ} \mathrm{C}$ under argon. $\mathrm{LiAlH}_{4}(0.20 \mathrm{~mL}, 1.0 \mathrm{M}$ in THF, $0.20 \mathrm{mmol})$ was added at $0{ }^{\circ} \mathrm{C}$, the reaction stirred for $2 \mathrm{~h}$, and then quenched with saturated aqueous Rochelle's salt (1.0 $\mathrm{mL})$. The layers were separated and the aqueous layer was extracted successively with ether $(3 \times 7 \mathrm{~mL})$, the combined organic layers were washed with brine $(3 \mathrm{~mL})$, dried $\left(\mathrm{Na}_{2} \mathrm{SO}_{4}\right)$, and then concentrated to yield an oil that was purified over basic alumina eluting with $2 \%$ triethylamine in $10 \%$ ether/petrol, yielding 4-cyclohexylidene-4-ethoxybutan-1-ol (S7) as a colorless oil (22 $\mathrm{mg}, 92 \%)$ that was taken directly into the next reaction. $\mathrm{R}_{f} 0.41$ (petrol/ether, $\left.2: 1\right)$; $\delta_{\mathrm{H}}\left(200 \mathrm{MHz}, \mathrm{C}_{6} \mathrm{D}_{6}\right) 1.09(3 \mathrm{H}, \mathrm{t}, J=7.0 \mathrm{~Hz})$, 1.30-1.78 (8 H, m), 2.00-2.11 (2 H, m), $2.19(2 \mathrm{H}, \mathrm{t}, J=7.0 \mathrm{~Hz}), 2.32-2.44(2 \mathrm{H}, \mathrm{m}), 3.32-3.50(4 \mathrm{H}$, $\mathrm{m})$. To a $7.0 \mathrm{~mL}$ sample vial was added alcohol $\mathbf{S} 7(20 \mathrm{mg}, 0.10 \mathrm{mmol})$ in DMF $(1.0 \mathrm{~mL})$ and the solution was cooled to $0{ }^{\circ} \mathrm{C}$ under argon. Freshly recystallised carbon tetrabromide $(66 \mathrm{mg}, 0.20 \mathrm{mmol})$ and triphenylphosphine $(52 \mathrm{mg}, 0.20 \mathrm{mmol})$ were added, leading the colorless solution to become yellow. After $0.5 \mathrm{~h}$ at $0{ }^{\circ} \mathrm{C}, \mathrm{NaN}_{3}(26 \mathrm{mg}, 0.40 \mathrm{mmol})$ was added and the reaction was stirred for $14 \mathrm{~h}$, during which the ice bath warmed from $0{ }^{\circ} \mathrm{C}$ to $15^{\circ} \mathrm{C}$. The cloudy brown solution was extracted with petrol $(8$ $\times 3 \mathrm{~mL}$ ) until no further product was observed by TLC analysis in the DMF layer. The combined petrol extracts were washed successively with saturated aqueous $\mathrm{NaHCO}_{3}(5 \mathrm{~mL})$ and brine $(5 \mathrm{~mL})$, then dried $\left(\mathrm{Na}_{2} \mathrm{SO}_{4}\right)$ and concentrated to yield an oil that was purified over basic alumina eluting with $1 \%$ triethylamine and 5\% ether/petrol, yielding the title compound as a colorless oil (13 mg, 58\%). $\mathrm{R}_{f} 0.88$ (petrol); $\delta_{\mathrm{H}}\left(400 \mathrm{MHz}, \mathrm{CDCl}_{3}\right) 1.08(3 \mathrm{H}, \mathrm{t}, J=7.0 \mathrm{~Hz}), 1.42-1.52(6 \mathrm{H}, \mathrm{m}), 1.99-2.12(4 \mathrm{H}, \mathrm{m}), 2.34-$ $2.36(2 \mathrm{H}, \mathrm{m}), 2.83(2 \mathrm{H}, \mathrm{t}, J=6.5 \mathrm{~Hz}), 3.39(2 \mathrm{H}, \mathrm{q}, J=7.0 \mathrm{~Hz}) ; \delta_{\mathrm{C}}\left(100 \mathrm{MHz}, \mathrm{C}_{6} \mathrm{D}_{6}\right)$ [from HSQC] $15.6,23.8,26.8,27.2,27.6,28.2,28.5,29.6,50.6,64.6,123.8,144.9$. 


\section{5-(1-Ethoxycyclohexyl)-3,4-dihydro-2H-pyrrole (21)}

To an oven-dried $14 \mathrm{~mL}$ Ace pressure tube was added azide 19 (6.0 mg, $0.027 \mathrm{mmol})$ and toluene- $d_{8}(1.0$ $\mathrm{mL}$ ). The reaction vessel was flushed with argon, the thread sealed with PTFE tape, and the screw cap with FETFE ${ }^{\circledR}$ O-ring fitted; the whole was wrapped in cotton wool/aluminum foil and the colorless solution was stirred at $130{ }^{\circ} \mathrm{C}$ for $84 \mathrm{~h}$. The tube was cooled to $\mathrm{RT}$, the brown reaction solution was filtered through a sintered glass funnel, washing through with ether, and the solution concentrated onto silica for chromatography on silica gel, eluting with $10 \% \rightarrow 40 \%$ ether/petrol. The volatile product was obtained as a $78 \% \mathrm{w} / \mathrm{w}$ solution in ether $(\sim 1.6 \mathrm{mg}, \sim 30 \%) . \mathrm{R}_{f} 0.61$ (ether); $\delta_{\mathrm{H}}\left(400 \mathrm{MHz}, \mathrm{CDCl}_{3}\right) 1.19(3$ $\mathrm{H}, \mathrm{t}, J=7.0 \mathrm{~Hz}), 1.48-1.74(8 \mathrm{H}, \mathrm{m}), 1.84-1.92(4 \mathrm{H}, \mathrm{m}), 2.59(2 \mathrm{H}, \mathrm{tt}, J=8.0,2.0 \mathrm{~Hz}), 3.21(2 \mathrm{H}, \mathrm{q}, J$ $=7.0 \mathrm{~Hz}), 3.89(2 \mathrm{H}, \mathrm{tt}, J=7.5,2.0 \mathrm{~Hz}) ; \mathrm{m} / z\left(\mathrm{ESI}^{+}\right) 196.2\left(\mathrm{MH}^{+}\right)$.

\section{Ethyl 4-cyclobutylidenebutanoate (S8); ${ }^{42}$ general procedure for Johnson-Claisen rearrangement}

To an oven-dried $50 \mathrm{~mL}$ round-bottomed flask equipped with stirrer bar, distillation head and thermometer was added triethylorthoacetate $(3.7 \mathrm{~mL}), 1$-vinylcyclobutanol ${ }^{35}(190 \mathrm{mg}, 1.94 \mathrm{mmol})$, and a few crystals of hydroquinone. The set-up was flushed with argon, then placed into an oil bath preheated to $140{ }^{\circ} \mathrm{C}$. Two drops of propionic acid were added and the mixture was stirred for $22 \mathrm{~h}$. Ethanol was observed distilling off at $78^{\circ} \mathrm{C}$. Reaction completion was indicated by TLC. The mixture was cooled to RT, diluted with ether $(50 \mathrm{~mL})$ and washed successively with hydrochloric acid $(0.1 \mathrm{M}, 20 \mathrm{~mL})$, saturated aqueous $\mathrm{NaHCO}_{3}$ solution $(10 \mathrm{~mL})$, and brine $(10 \mathrm{~mL})$. The organic layer was dried $\left(\mathrm{MgSO}_{4}\right)$ and concentrated to afford an oil that was purified over silica gel, eluting with petrol, to yield a colorless oil (253 mg, 78\%). $\mathrm{R}_{f} 0.60$ (petrol/ether, 3:1); $v_{\max } 1735 \mathrm{~s}, 1164 \mathrm{~s} ; \delta_{\mathrm{H}}\left(400 \mathrm{MHz}, \mathrm{CDCl}_{3}\right) 1.26(3 \mathrm{H}, \mathrm{t}, J=$ $7.0 \mathrm{~Hz}), 1.92(2 \mathrm{H}$, app. quin, $J=8.0 \mathrm{~Hz}), 2.18(2 \mathrm{H}$, app. q, $J=7.0 \mathrm{~Hz}), 2.31(2 \mathrm{H}, \mathrm{t}, J=7.0 \mathrm{~Hz}), 2.54-$ $2.59(4 \mathrm{H}, \mathrm{m}), 4.13(2 \mathrm{H}, \mathrm{q}, J=8.0 \mathrm{~Hz}), 4.98-5.05(1 \mathrm{H}, \mathrm{m}) ; \delta_{\mathrm{C}}\left(100 \mathrm{MHz}, \mathrm{CDCl}_{3}\right)$ 14.4, 17.1, 23.7, 29.3, 
31.0, 34.6, 60.3, 118.3, 141.7, 173.6; HRMS $\left(\mathrm{FI}^{+}\right) \mathrm{m} / \mathrm{z}:\left[\mathrm{M}^{+}\right]$Calcd for $\mathrm{C}_{10} \mathrm{H}_{16} \mathrm{O}_{2}$ 168.1145; Found 168.1148.

\section{Ethyl 4-cyclopentylidenebutanoate (S9) ${ }^{43}$}

Application of the general procedure described above (for S8) afforded ester S9 (1.25 g, 61\% from 1vinylcyclopentanol ${ }^{36}$ ) as a colorless oil. $\mathrm{R}_{f} 0.82$ (petrol/ether, $\left.3: 1\right) ; v_{\max } 1736 \mathrm{~s} ; \delta_{\mathrm{H}}\left(400 \mathrm{MHz}, \mathrm{CDCl}_{3}\right)$ $1.26(3 \mathrm{H}, \mathrm{t}, J=7.0 \mathrm{~Hz}), 1.57-1.69(4 \mathrm{H}, \mathrm{m}), 2.20$ (4 H, app. q, $J=7.0 \mathrm{~Hz}), 2.28-2.36(4 \mathrm{H}, \mathrm{m}), 4.11(2$ $\mathrm{H}, \mathrm{q}, J=7.0 \mathrm{~Hz}), 5.17-5.23(1 \mathrm{H}, \mathrm{m}) ; \delta_{\mathrm{C}}\left(100 \mathrm{MHz}, \mathrm{CDCl}_{3}\right)$ 14.4, 25.3, 26.4, 26.5, 28.7, 33.7, 34.5, 60.3, 118.0, 144.9, 173.7; $m / z\left(\mathrm{ESI}^{+}\right) 205.1\left(\mathrm{MNa}^{+}\right), 183.1\left(\mathrm{MH}^{+}\right)$.

\section{4-Cyclobutylidenebutan-1-ol (S10); general procedure for ester reduction}

To an oven-dried $10 \mathrm{~mL}$ round-bottomed flask was added ester $\mathbf{S 8}$ (230 mg, $1.37 \mathrm{mmol})$ in THF (1.0 $\mathrm{mL})$ and the solution was stirred at $0{ }^{\circ} \mathrm{C}$ under argon. $\mathrm{LiAlH}_{4}(1.5 \mathrm{~mL}, 1.0 \mathrm{M}$ in $\mathrm{THF}, 1.5 \mathrm{mmol})$ was added dropwise via syringe over $1 \mathrm{~min}$, and the reaction was stirred for $13 \mathrm{~h}$ during which the ice bath warmed to $15^{\circ} \mathrm{C}$. The reaction was quenched carefully with saturated aqueous Rochelle's salt (1 mL) then brine $(10 \mathrm{~mL})$, and the mixture was extracted with ether $(3 \times 20 \mathrm{~mL})$. The combined organic extracts were washed successively with saturated aqueous $\mathrm{NaHCO}_{3}$ solution $(10 \mathrm{~mL})$, then brine $(10 \mathrm{~mL})$, dried $\left(\mathrm{Na}_{2} \mathrm{SO}_{4}\right)$, and concentrated to yield a colorless oil (168 mg, 97\%). $\mathrm{R}_{f} 0.37$ (petrol/ether, 1:1); $v_{\max }$ $3327 \mathrm{br} ; \delta_{\mathrm{H}}\left(400 \mathrm{MHz}, \mathrm{CDCl}_{3}\right) 1.60(2 \mathrm{H}$, app. quin, $J=6.5 \mathrm{~Hz}), 1.89-1.99(4 \mathrm{H}, \mathrm{m}), 2.61-2.66(4 \mathrm{H}, \mathrm{m})$, $3.64(2 \mathrm{H}, \mathrm{t}, J=6.5 \mathrm{~Hz}), 5.04-5.09(1 \mathrm{H}, \mathrm{m}) ; \delta_{\mathrm{C}}\left(100 \mathrm{MHz}, \mathrm{CDCl}_{3}\right)$ 17.1, 24.5, 29.4, 31.0, 32.7, 62.9,

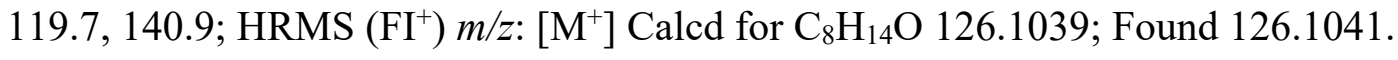

\section{4-Cyclopentylidenebutan-1-ol (S11)}




\begin{abstract}
Application of the general procedure described above (for S10) afforded alcohol S11 (851 mg, 92\% from ester S9) as a colorless oil. $\mathrm{R}_{f} 0.32$ (petrol/ether, 1:1); $v_{\max } 3331 \mathrm{br} ; \delta_{\mathrm{H}}\left(400 \mathrm{MHz}, \mathrm{CDCl}_{3}\right) 1.55-1.69$ (6 H, m), 2.05 (2 H, app. q, $J=7.0 \mathrm{~Hz}), 2.16-2.23(4 \mathrm{H}, \mathrm{m}), 3.64(2 \mathrm{H}, \mathrm{t}, J=6.5 \mathrm{~Hz}), 5.20-5.27(1 \mathrm{H}, \mathrm{m})$; $\delta_{\mathrm{C}}\left(100 \mathrm{MHz}, \mathrm{CDCl}_{3}\right)$ 26.1, 26.5 (two peaks), 28.7, 32.7, 33.7, 62.9, 119.4, 144.1; m/z $\left(\mathrm{ESI}^{+}\right) 141.2$ $\left(\mathrm{MH}^{+}\right)$.
\end{abstract}

\title{
6-Azido-2-methylhex-2-ene (S12);34 general procedure for mesylation/azide displacement
}

To an oven-dried $100 \mathrm{~mL}$ round-bottomed flask was added 5-methylhex-4-en-1-ol ${ }^{34}$ (948 mg, 8.30 $\mathrm{mmol})$, ether $(20 \mathrm{~mL})$ and triethylamine $(1.40 \mathrm{~mL}, 10.0 \mathrm{mmol})$ and the solution was cooled to $0{ }^{\circ} \mathrm{C}$ under argon. Methanesulfonyl chloride $(0.80 \mathrm{~mL}, 10.3 \mathrm{mmol})$ was added dropwise via syringe over $5 \mathrm{~min}$, causing a white precipitate to form. After $0.5 \mathrm{~h}$, the reaction mixture was warmed to RT and stirred for $3 \mathrm{~h}$. The reaction was quenched with brine $(15 \mathrm{~mL})$, and the separated aqueous layer extracted with ether $(3 \times 15 \mathrm{~mL})$. The organic extracts were washed with brine $(5 \mathrm{~mL})$, dried $\left(\mathrm{Na}_{2} \mathrm{SO}_{4}\right)$ and concentrated to yield a colorless oil (the mesylate) that was used directly in the next reaction. The crude mesylate was transferred to a $50 \mathrm{~mL}$ round-bottomed flask with DMSO $(4 \mathrm{~mL})$ and $\mathrm{NaN}_{3}(1.0 \mathrm{~g}, 15.4 \mathrm{mmol})$, and the mixture was stirred at $35^{\circ} \mathrm{C}$ for $18 \mathrm{~h}$. The product was extracted into petrol $(80 \mathrm{~mL})$ and the petrol layer was washed with water $(4 \times 10 \mathrm{~mL})$. The combined aqueous washes were extracted with petrol $(3 \times 10$ $\mathrm{mL})$, then the combined organic portions were dried $\left(\mathrm{MgSO}_{4}\right)$ and concentrated. The crude product was purified over silica gel, eluting with petrol to yield the title compound (S12) as a colorless oil (900 mg, $78 \%$ over the two steps). $\mathrm{R}_{f} 0.76$ (petrol); $v_{\max } 2093 \mathrm{~s} ; \delta_{\mathrm{H}}\left(400 \mathrm{MHz}, \mathrm{CDCl}_{3}\right) 1.62(3 \mathrm{H}, \mathrm{s}), 1.64(2 \mathrm{H}$, app. quin, $J=7.0 \mathrm{~Hz}), 1.71(3 \mathrm{H}, \mathrm{d}, J=1.0 \mathrm{~Hz}), 2.08(2 \mathrm{H}$, app. q, $J=7.0 \mathrm{~Hz}), 3.27(2 \mathrm{H}, \mathrm{t}, J=7.0 \mathrm{~Hz})$, $5.09(1 \mathrm{H}$, t. hept, $J=7.0,1.0 \mathrm{~Hz}) ; \delta_{\mathrm{C}}\left(100 \mathrm{MHz}, \mathrm{CDCl}_{3}\right) 17.8,25.2,25.8,29.0,51.1,123.0,133.0$; HRMS $\left(\mathrm{FI}^{+}\right) m / z:\left[\mathrm{M}^{+}\right]$Calcd for $\mathrm{C}_{7} \mathrm{H}_{13} \mathrm{~N}_{3}$ 139.1104; Found 139.1106. 


\section{(4-Azidobutylidene)cyclobutane (S13)}

Application of the general procedure described above (for S12) afforded azide S13 (119 mg, 70\% from alcohol S10) as a colorless oil. $\mathrm{R}_{f} 0.40$ (petrol); $v_{\max } 2093 \mathrm{~s} ; \delta_{\mathrm{H}}\left(400 \mathrm{MHz}, \mathrm{CDCl}_{3}\right) 1.62(2 \mathrm{H}$, app. quin, $J=7.0 \mathrm{~Hz}), 1.90-2.00(4 \mathrm{H}, \mathrm{m}), 2.61-2.67(4 \mathrm{H}, \mathrm{m}), 3.26(2 \mathrm{H}, \mathrm{t}, J=7.0 \mathrm{~Hz}), 4.97-5.05(1 \mathrm{H}, \mathrm{m}) ; \delta_{\mathrm{C}}$

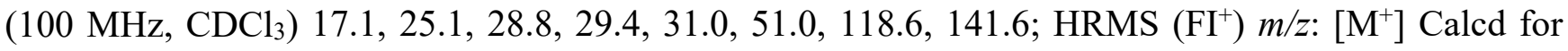
$\mathrm{C}_{8} \mathrm{H}_{13} \mathrm{~N}_{3}$ 151.1104; Found 151.1113.

\section{(4-Azidobutylidene)cyclopentane (S14)}

Application of the general procedure described above (for S12) afforded azide S14 as a colorless oil (711 $\mathrm{mg}, 77 \%$ from alcohol S11). $\mathrm{R}_{f} 0.38$ (petrol); $v_{\max } 2090 \mathrm{~s} ; \delta_{\mathrm{H}}\left(400 \mathrm{MHz}, \mathrm{CDCl}_{3}\right) 1.59-1.69(6 \mathrm{H}, \mathrm{m}), 2.07$ $(2 \mathrm{H}, \mathrm{qt}, J=7.5,1.5 \mathrm{~Hz}), 2.16-2.25(4 \mathrm{H}, \mathrm{m}), 3.27(2 \mathrm{H}, \mathrm{t}, J=7.0 \mathrm{~Hz}), 5.16-5.23(1 \mathrm{H}, \mathrm{m}) ; \delta_{\mathrm{C}}(100 \mathrm{MHz}$, $\mathrm{CDCl}_{3}$ ) 26.5 (two peaks), 26.7, 28.8 (two peaks), 33.7, 51.1, 118.4, 144.8; $\mathrm{HRMS}\left(\mathrm{FI}^{+}\right) \mathrm{m} / z$ : $\left[\mathrm{M}^{+}\right] \mathrm{Calcd}$ for $\mathrm{C}_{9} \mathrm{H}_{15} \mathrm{~N}_{3}$ 165.1260; Found 165.1254.

\section{6-Azido-3-bromo-2-methylhex-2-ene (22a); general procedure for bromination/dehydrobromination of trisubstituted alkenes}

To an oven-dried $50 \mathrm{~mL}$ round-bottomed flask was added 6-azido-2-methylhex-2-ene $\mathbf{S 1 2}$ (420 mg, 3.02 $\mathrm{mmol})$ and petrol $(10 \mathrm{~mL})$, and the solution was cooled to $0{ }^{\circ} \mathrm{C}$ under argon. A solution of $\mathrm{Br}_{2}(0.16 \mathrm{~mL}$, $3.12 \mathrm{mmol})$ in petrol $(0.5 \mathrm{~mL})$ was added dropwise to the reaction via syringe over $15 \mathrm{~min}$, causing an orange coloration that subsided and then persisted. The mixture was warmed to RT, stirred for $5 \mathrm{~min}$, then cooled to $0{ }^{\circ} \mathrm{C}$ and quenched with saturated aqueous $\mathrm{Na}_{2} \mathrm{~S}_{2} \mathrm{O}_{3}$ solution $(30 \mathrm{~mL})$. After the orange color faded to colorless, the layers were separated and the aqueous extracted with petrol $(3 \times 30 \mathrm{~mL})$, the combined organic extracts were washed with brine $(5 \mathrm{~mL})$, dried $\left(\mathrm{MgSO}_{4}\right)$, and concentrated to yield a 
yellow oil. The crude product was transferred to a $50 \mathrm{~mL}$ round-bottomed flask with DMSO (1.0 mL) and stirred at RT under argon. Potassium tert-butoxide (4.5 mL, 1.0 M in tert-butanol, $4.5 \mathrm{mmol})$ was added dropwise via syringe pump over $20 \mathrm{~min}$, causing the colorless solution to become brown, and stirring was continued for a further $14 \mathrm{~h}$. The reaction mixture was concentrated to half its volume under vacuum to remove tert-butanol, then was diluted with saturated aqueous $\mathrm{NaHCO}_{3}$ solution $(20 \mathrm{~mL})$. The solution was extracted with ether $(4 \times 20 \mathrm{~mL})$, the combined organics were washed with brine $(10 \mathrm{~mL})$, dried $\left(\mathrm{MgSO}_{4}\right)$, and concentrated to yield a brown oil. The crude product was purified over silica gel, eluting with petrol to yield the title compound as a pale yellow oil ( $0.49 \mathrm{~g}, 74 \%$ over two steps). $\mathrm{R}_{f} 0.31$ (petrol); $v_{\max } 2092 \mathrm{~s} ; \delta_{\mathrm{H}}\left(400 \mathrm{MHz}, \mathrm{CDCl}_{3}\right) 1.80(3 \mathrm{H}, \mathrm{s}), 1.85(2 \mathrm{H}$, app. quin, $J=7.0 \mathrm{~Hz}), 1.89(3 \mathrm{H}, \mathrm{s})$, $2.60(2 \mathrm{H}, \mathrm{t}, J=7.0 \mathrm{~Hz}), 3.30(2 \mathrm{H}, \mathrm{t}, J=7.0 \mathrm{~Hz}) ; \delta_{\mathrm{C}}\left(100 \mathrm{MHz}, \mathrm{CDCl}_{3}\right) 20.5,25.5,27.5,34.4,50.3$, 120.1, 131.8; HRMS (FI') m/z: $\left[\mathrm{M}^{+}\right]$Calcd for $\mathrm{C}_{7} \mathrm{H}_{12}{ }^{79} \mathrm{BrN}_{3} 217.0209$; Found 217.0131.

\section{(4-Azido-1-bromobutylidene)cyclobutane (22b); alternative general procedure for} bromination/dehydrobromination of trisubstituted alkenes

To an oven-dried $25 \mathrm{~mL}$ round-bottomed flask was added azide $\mathbf{S 1 3}$ (330 mg, $2.18 \mathrm{mmol}$ ) and dichloromethane $(6.0 \mathrm{~mL})$, and the solution was cooled to $0{ }^{\circ} \mathrm{C}$ under argon. A solution of $\mathrm{Br}_{2}(0.12 \mathrm{~mL}$, $2.34 \mathrm{mmol})$ in petrol $(1.0 \mathrm{~mL})$ was added dropwise to the reaction via syringe over $1 \mathrm{~min}$, causing an orange coloration that subsided and then persisted. The mixture was stirred at $0{ }^{\circ} \mathrm{C}$ for 5 min, then warmed to RT. After stirring for $0.5 \mathrm{~h}$, the reaction mixture was concentrated under vaccum to yield an orange oil. To the crude dibromide was added THF $(5.0 \mathrm{~mL})$, and the solution was cooled to $-78{ }^{\circ} \mathrm{C}$ under argon. KHMDS (7.2 mL, 0.91 M in THF, $6.6 \mathrm{mmol})$ was added dropwise via syringe pump over 20 min causing the clear orange solution to become dark brown, and the mixture was stirred for a further $14 \mathrm{~h}$ during which the cooling bath warmed from $-78^{\circ} \mathrm{C}$ to $\mathrm{RT}$. The reaction was quenched with saturated aqueous $\mathrm{NH}_{4} \mathrm{Cl}$ solution $(50 \mathrm{~mL})$ and the product was extracted into petrol $(3 \times 20 \mathrm{~mL})$. The combined 
organic extracts were washed successively with saturated aqueous $\mathrm{NaHCO}_{3}$ solution $(5 \mathrm{~mL})$, brine (5 $\mathrm{mL})$, then dried $\left(\mathrm{MgSO}_{4}\right)$, and concentrated to yield a brown oil. The crude product was purified over silica gel, eluting with petrol to yield a colorless oil (282 mg, $56 \%$ over two steps). $\mathrm{R}_{f} 0.31$ (petrol); $v_{\max }$ 2096s; $\delta_{\mathrm{H}}\left(400 \mathrm{MHz}, \mathrm{CDCl}_{3}\right) 1.82(2 \mathrm{H}$, app. quin, $J=7.0 \mathrm{~Hz}), 1.94(2 \mathrm{H}$, app. quin, $J=7.0 \mathrm{~Hz}), 2.38$ $(2 \mathrm{H}, \mathrm{tt}, J=7.0,1.0 \mathrm{~Hz}), 2.60-2.69(4 \mathrm{H}, \mathrm{m}), 3.30(2 \mathrm{H}, \mathrm{t}, J=7.0 \mathrm{~Hz}) ; \delta_{\mathrm{C}}\left(100 \mathrm{MHz}, \mathrm{CDCl}_{3}\right) 15.1,27.0$, 29.7, 32.2, 32.3, 115.1, 140.5; HRMS $\left(\mathrm{FI}^{+}\right) \mathrm{m} / z$ : $\left[\mathrm{M}^{+}\right]$Calcd for $\mathrm{C}_{8} \mathrm{H}_{12}{ }^{79} \mathrm{BrN}_{3}$ 229.0209; Found 229.0215

\section{(4-Azido-1-bromobutylidene)cyclopentane (22c)}

Application of the general procedure described above (for 22a) afforded bromide 22c (279 mg, $75 \%$ from azide S14) as a colorless oil. $\mathrm{R}_{f} 0.17$ (petrol); $v_{\max } 2094 \mathrm{~s} ; \delta_{\mathrm{H}}\left(400 \mathrm{MHz}, \mathrm{CDCl}_{3}\right) 1.69$ (2 H, app. quin, $J$ $=7.0 \mathrm{~Hz}), 1.78(2 \mathrm{H}$, app. quin, $J=7.0 \mathrm{~Hz}), 1.85(2 \mathrm{H}$, app. quin, $J=6.5 \mathrm{~Hz}), 2.30(2 \mathrm{H}, \mathrm{t}, J=7.0 \mathrm{~Hz})$, $2.34(2 \mathrm{H}, \mathrm{t}, J=7.0 \mathrm{~Hz}), 2.53(2 \mathrm{H}, \mathrm{t}, J=7.0 \mathrm{~Hz}), 3.30(2 \mathrm{H}, \mathrm{t}, J=6.5 \mathrm{~Hz}) ; \delta_{\mathrm{C}}\left(100 \mathrm{MHz}, \mathrm{CDCl}_{3}\right) 26.1$, 27.3, 28.0, 32.0, 35.2, 36.1, 50.3, 115.7, 144.3; HRMS $\left(\mathrm{FI}^{+}\right) \mathrm{m} / z$ : $\left[\mathrm{M}-\mathrm{Br}-\mathrm{N}_{2}\right]^{+}$Calcd for $\mathrm{C}_{9} \mathrm{H}_{14} \mathrm{~N}$ 136.1121; Found 136.1135.

\section{(4-Azidobutylidene)cyclohexane (S15) and (4-azido-1-bromobutylidene)cyclohexane (22d)}

Application of the general procedure described above (for S12) afforded crude azide S15 (from 4cyclohexylidenebutan-1-ol ${ }^{37}$ ) as a pale brown oil that was taken directly into the next reaction. $\mathrm{R}_{f} 0.40$ (petrol); $v_{\max } 2092 \mathrm{~s} ; \delta_{\mathrm{H}}\left(400 \mathrm{MHz}, \mathrm{CDCl}_{3}\right) 1.47-1.58(6 \mathrm{H}, \mathrm{m}), 1.63(2 \mathrm{H}$, app. quin, $J=7.0 \mathrm{~Hz}), 2.06-$ $2.14(6 \mathrm{H}, \mathrm{m}), 3.27(2 \mathrm{H}, \mathrm{t}, J=7.0 \mathrm{~Hz}), 5.04(1 \mathrm{H}, \mathrm{t}, J=7.0) ; \delta_{\mathrm{C}}\left(100 \mathrm{MHz}, \mathrm{CDCl}_{3}\right) 24.2,27.0,28.0$, 28.8 (two peaks), 29.3, 37.3, 51.0, 119.5, 141.3; $\mathrm{HRMS}\left(\mathrm{FI}^{+}\right) \mathrm{m} / z:\left[\mathrm{M}-\mathrm{N}_{2}\right]^{+}$Calcd for $\mathrm{C}_{10} \mathrm{H}_{17} \mathrm{~N} 151.1356$; Found 151.1336. This crude azide was then taken through the general procedure described above (for 22a) to yield the product as a pale yellow oil (11.0 g, 52\% from 4-cyclohexylidenebutan-1-ol). $\mathrm{R}_{f} 0.21$ (petrol); $v_{\max } 2093 \mathrm{~s} ; \delta_{\mathrm{H}}\left(400 \mathrm{MHz}, \mathrm{CDCl}_{3}\right) 1.56(6 \mathrm{H}$, br. s), $1.85(2 \mathrm{H}$, app. quin, $J=7.0 \mathrm{~Hz}), 2.25-2.28$ 


\begin{abstract}
(2 H, m), $2.40\left(2 \mathrm{H}\right.$, br. s), $2.63(2 \mathrm{H}, \mathrm{t}, J=7.0 \mathrm{~Hz}), 3.30(2 \mathrm{H}, \mathrm{t}, J=6.5 \mathrm{~Hz}) ; \delta_{\mathrm{C}}\left(100 \mathrm{MHz}, \mathrm{CDCl}_{3}\right) 26.5$, 27.4, 27.5, 28.0, 31.3, 34.0, 35.6, 50.2, 117.6, 139.4; HRMS $\left(\mathrm{FI}^{+}\right) m / z:\left[\mathrm{M}^{+}\right]$Calcd for $\mathrm{C}_{10} \mathrm{H}_{16}{ }^{79} \mathrm{BrN}_{3}$ 257.0522; Found 257.0543.
\end{abstract}

\title{
Dimethyl 2-(4-azido-1-hydroxybutylidene)malonate (S16)
}

To a $250 \mathrm{~mL}$ oven-dried round-bottomed flask was added anhydrous $\mathrm{MgCl}_{2}(1.70 \mathrm{~g}, 17.9 \mathrm{mmol})$ and acetonitrile $(100 \mathrm{~mL})$ at $0{ }^{\circ} \mathrm{C}$ under argon. Dimethyl malonate $(1.80 \mathrm{~mL}, 15.8 \mathrm{mmol})$ and triethylamine (4.40 mL, $31.6 \mathrm{mmol})$ were added dropwise, followed $10 \mathrm{~min}$ later by a solution of 4-azidobutyryl chloride $^{40}(3.48 \mathrm{~g}, 23.6 \mathrm{mmol})$ in acetonitrile $(3.0 \mathrm{~mL})$, dropwise over $45 \mathrm{~min}$. The mixture was stirred at $0{ }^{\circ} \mathrm{C}$ for $1 \mathrm{~h}$ then warmed to ambient temperature, and, after $1 \mathrm{~h}$ stirring, quenched with water $(150$ $\mathrm{mL})$ and extracted with ether $(3 \times 200 \mathrm{~mL})$; the combined extracts were washed with brine $(20 \mathrm{~mL})$, dried $\left(\mathrm{MgSO}_{4}\right)$, concentrated, and purified on silica gel, eluting with $10 \%$ ether/petrol to yield the title compound as a ca. 60:40 mixture of keto/enol tautomers and as a pale yellow oil (2.66 g, 69\%). $\mathrm{R}_{f} 0.40$ (petrol/ether, 1:1); $v_{\max } 2097 \mathrm{~s}, 1722 \mathrm{~s}, 1651 \mathrm{~m}, 1602 \mathrm{~m} ; \delta_{\mathrm{H}}\left(400 \mathrm{MHz}, \mathrm{CDCl}_{3}\right)$ [keto/enol mixture] 1.87$1.97(2 \mathrm{H}, \mathrm{m}), 2.55(0.8 \mathrm{H}, \mathrm{t}, J=7.5 \mathrm{~Hz}), 2.73(1.2 \mathrm{H}, \mathrm{t}, J=7.0 \mathrm{~Hz}), 3.32-3.37(2 \mathrm{H}, \mathrm{m}), 3.79(1.2 \mathrm{H}, \mathrm{s})$, $3.81(3.6 \mathrm{H}, \mathrm{s}), 3.82(1.2 \mathrm{H}, \mathrm{s}), 4.51(0.6 \mathrm{H}, \mathrm{s}), 13.50(0.4 \mathrm{H}, \mathrm{s}) ; \delta_{\mathrm{C}}\left(100 \mathrm{MHz}, \mathrm{CDCl}_{3}\right)$ [keto/enol mixture] $22.9,26.0,31.1,38.7,50.4,50.7,52.3,52.5,53.4,53.8,65.0,99.8,165.0,166.6,171.6,182.2,197.9$; HRMS $\left(\mathrm{ESI}^{+}\right) \mathrm{m} / z$ : $[\mathrm{M}+\mathrm{Na}]^{+}$Calcd for $\mathrm{C}_{9} \mathrm{H}_{13} \mathrm{~N}_{3} \mathrm{NaO}_{5}$ 266.0747; Found 266.0755.

\section{Dimethyl 2-(4-azido-1-bromobutylidene)malonate (22e)}

To a $50 \mathrm{~mL}$ oven-dried round-bottomed flask was added DMF $(3.4 \mathrm{~mL})$ and the solution was stirred at 0 ${ }^{\circ} \mathrm{C}$ under argon. $\mathrm{PBr}_{3}(0.25 \mathrm{~mL}, 2.63 \mathrm{mmol})$ in ether $(2.0 \mathrm{~mL})$ was added dropwise over 40 min leading to a white precipitate to form. After $10 \mathrm{~min}$, a solution of malonate derivative $\mathbf{S 1 6}(200 \mathrm{mg}, 0.822 \mathrm{mmol})$ in DMF (1.0 mL) was added over $25 \mathrm{~min}$, washing in with further DMF (1.0 mL). An oven-dried reflux 
condenser was attached under argon, and the reaction mixture was heated to $45^{\circ} \mathrm{C}$ for $22 \mathrm{~h}$, during which time the white precipitate became green. The flask was removed from the heat, quenched with brine ( 60 $\mathrm{mL})$, extracted with ether $(3 \times 20 \mathrm{~mL})$, and the combined extracts were washed with brine $(10 \mathrm{~mL})$, dried $\left(\mathrm{MgSO}_{4}\right)$, and concentrated. The crude product was purified over silica gel, eluting with $6 \%$ ether/petrol, yielding a yellow oil (83 mg, 33\%). $\mathrm{R}_{f} 0.50$ (petrol/ether, 1:1); $v_{\max } 2098 \mathrm{~s}, 1729 \mathrm{~s}, 1621 \mathrm{~m} ; \delta_{\mathrm{H}}(400 \mathrm{MHz}$, $\left.\mathrm{CDCl}_{3}\right) 1.96(2 \mathrm{H}$, app. quin, $J=7.0 \mathrm{~Hz}), 3.19(2 \mathrm{H}$, app. t, $J=7.5 \mathrm{~Hz}), 3.38(2 \mathrm{H}, \mathrm{t}, J=7.0 \mathrm{~Hz}), 3.79(3$ $\mathrm{H}, \mathrm{s}), 3.86(3 \mathrm{H}, \mathrm{s}) ; \delta_{\mathrm{C}}\left(100 \mathrm{MHz}, \mathrm{CDCl}_{3}\right) 27.7,36.3,50.4,52.9,53.0,129.9,146.6,162.2,165.4$; HRMS $\left(\mathrm{ESI}^{+}\right) \mathrm{m} / z:[\mathrm{M}+\mathrm{Na}]^{+}$Calcd for $\mathrm{C}_{9} \mathrm{H}_{12}{ }^{79} \mathrm{BrN}_{3} \mathrm{NaO}_{4}$ 327.9903; Found 327.9910 .

\section{6-Azido-2,5,5-trimethylhex-2-ene (S17)}

To an oven-dried $25 \mathrm{~mL}$ round-bottomed flask was added 2,2,5-trimethylhex-4-en-1-ol ${ }^{39}$ (500 mg, 3.52 $\mathrm{mmol})$, pyridine $(0.50 \mathrm{ml}, 6.18 \mathrm{mmol})$ and dry dichloromethane $(5.0 \mathrm{~mL})$. The flask was cooled to -78 ${ }^{\circ} \mathrm{C}$ under argon and trifluoromethanesulfonic acid anhydride $(0.88 \mathrm{~mL}, 5.23 \mathrm{mmol})$ was added dropwise; the mixture was stirred for $10 \mathrm{~min}$, then the cold bath was removed to allow the mixture to reach RT during which time the solution turned from yellow to pink to purple. TLC analysis after $1.5 \mathrm{~h}$ revealed the reaction to be complete. The reaction was quenched with brine $(50 \mathrm{~mL})$, the mixture was extracted with ether $(3 \times 20 \mathrm{~mL})$, and the combined organic extracts were washed with saturated aqueous $\mathrm{NaHCO}_{3}$ solution $(3 \times 2 \mathrm{~mL})$, then dried $\left(\mathrm{Na}_{2} \mathrm{SO}_{4}\right)$ and concentrated to yield the product as a red oil that was used directly in the next step. The crude triflate was dissolved in DMF (2.0 mL) and stirred with $\mathrm{NaN}_{3}(340$ $\mathrm{mg}, 5.23 \mathrm{mmol})$ under argon at $30^{\circ} \mathrm{C}$ overnight. The mixture was cooled to RT, diluted with ether (100 $\mathrm{mL})$, and washed successively with water $(10 \times 1 \mathrm{~mL})$ and brine $(1 \mathrm{~mL})$, then dried $\left(\mathrm{Na}_{2} \mathrm{SO}_{4}\right)$ and concentrated to yield the product as an oil that was sufficiently pure to use in the next step (313 mg, 53\% from the starting alcohol). $\mathrm{R}_{f} 0.58$ (petrol); $v_{\max } 2965 \mathrm{~m}, 2928 \mathrm{~m}, 2096 \mathrm{~s}, 1470 \mathrm{~m}, 1365 \mathrm{~m}, 845 \mathrm{w} ; \delta_{\mathrm{H}}(400$ $\left.\mathrm{MHz}, \mathrm{CDCl}_{3}\right) 0.91(6 \mathrm{H}, \mathrm{s}), 1.62$ and $1.74(2 \times 3 \mathrm{H}, 2 \times \mathrm{s}), 1.94(2 \mathrm{H}, \mathrm{d}, J=8.0 \mathrm{~Hz}), 3.09(2 \mathrm{H}, \mathrm{s}), 5.14$ 


\begin{abstract}
$(1 \mathrm{H}, \mathrm{tt}, J=8.0,1.5 \mathrm{~Hz}) ; \delta_{\mathrm{C}}\left(100 \mathrm{MHz}, \mathrm{CDCl}_{3}\right) 18.0,25.0,26.2,36.5,37.9,62.3,120.0,134.2$; HRMS
$\left(\mathrm{ESI}^{+}\right) \mathrm{m} / z:\left[\mathrm{M}+\mathrm{H}-\mathrm{N}_{2}\right]^{+}$Calcd for $\mathrm{C}_{9} \mathrm{H}_{18} \mathrm{~N}$ 140.1434; Found 140.1434.
\end{abstract}

\title{
6-Azido-3-bromo-2,5,5-trimethylhex-2-ene (22f)
}

To an oven-dried $50 \mathrm{~mL}$ round-bottomed flask was added azide $\mathbf{S 1 7}$ (1.12 g, $6.69 \mathrm{mmol})$ and dry dichloromethane $(7.0 \mathrm{~mL})$, and the flask was cooled to $-78^{\circ} \mathrm{C}$ under argon. A solution of $\operatorname{Br}_{2}(0.34 \mathrm{~mL}$, $6.64 \mathrm{mmol})$ in dichloromethane $(1 \mathrm{~mL})$ was added dropwise until the brown color remained. After stirring for $2 \mathrm{~h}$, the cooling bath was removed and the flask was allowed to warm to RT for $0.5 \mathrm{~h}$. The mixture was concentrated to an orange oil and purified over silica gel eluting with petrol, yielding 1-azido-4,5dibromo-2,2,5-trimethylhexane as a yellow oil (470 mg, 21\%). $\mathrm{R}_{f} 0.36$ (petrol); $v_{\max } 2964 \mathrm{w}, 2100 \mathrm{~s}$, $1457 \mathrm{w}, 1096 \mathrm{~m}, 805 \mathrm{w} ; \delta_{\mathrm{H}}\left(500 \mathrm{MHz}, \mathrm{CDCl}_{3}\right) 1.06$ and $1.08(2 \times 3 \mathrm{H}, 2 \times \mathrm{s}), 1.81$ and $2.00(2 \times 3 \mathrm{H}, 2 \times$ s), $2.07(1 \mathrm{H}, \mathrm{dd}, J=16.0,8.5 \mathrm{~Hz}), 2.50(1 \mathrm{H}, \mathrm{dd}, J=16.0,1.0 \mathrm{~Hz}), 3.24$ and $3.32(2 \times 1 \mathrm{H}, 2 \times \mathrm{d}, J=$ $12.0 \mathrm{~Hz}), 4.30(1 \mathrm{H}, \mathrm{d}, J=8.5 \mathrm{~Hz}) ; \delta_{\mathrm{C}}\left(125 \mathrm{MHz} \mathrm{CDCl}_{3}\right)$ 24.9, 25.5, 27.7, 35.2, 45.5, 59.8, 62.2, 69.5;

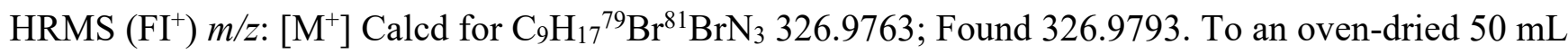
round-bottomed flask was added a sample of this dibromide (116 mg, $0.355 \mathrm{mmol})$ and dry THF (6.0 $\mathrm{mL}$ ), and the flask was cooled to $0{ }^{\circ} \mathrm{C}$ under argon. KHMDS (4.0 mL, 0.5 $\mathrm{M}$ in toluene, $2.0 \mathrm{mmol}$ ) was added dropwise over $1 \mathrm{~h}$, and the mixture was stirred for $14 \mathrm{~h}$ during which time the ice bath warmed to $10{ }^{\circ} \mathrm{C}$. The reaction was quenched with brine $(5 \mathrm{~mL})$ and the mixture was extracted with petrol $(3 \times 20$ $\mathrm{mL}$ ); the combined organic extracts were washed sequentially with saturated aqueous $\mathrm{NaHCO}_{3}$ solution $(10 \mathrm{~mL})$ and brine $(10 \mathrm{~mL})$, then dried $\left(\mathrm{Na}_{2} \mathrm{SO}_{4}\right)$ and concentrated to yield a brown oil. The crude product was purified over silica gel eluting with petrol, yielding the title compound (22f) as a colorless oil (53 $\mathrm{mg}, 61 \%$ ). $\mathrm{R}_{f} 0.40$ (petrol); $v_{\max } 2925 \mathrm{~m}, 2101 \mathrm{~s}, 1457 \mathrm{w}, 1389 \mathrm{w}, 802 \mathrm{w} ; \delta_{\mathrm{H}}\left(400 \mathrm{MHz}, \mathrm{CDCl}_{3}\right) 1.01(6 \mathrm{H}$, s), 1.78 and $1.91(2 \times 3 \mathrm{H}, 2 \times \mathrm{s}), 2.60(2 \mathrm{H}, \mathrm{s}), 3.25(2 \mathrm{H}, \mathrm{s}) ; \delta_{\mathrm{C}}\left(100 \mathrm{MHz}, \mathrm{CDCl}_{3}\right) 21.8,25.7,26.1$, 37.9, 46.1, 62.4, 117.1, 134.3; HRMS (FI') m/z: $\left[\mathrm{M}^{+}\right]$Calcd for $\mathrm{C}_{9} \mathrm{H}_{16}{ }^{79} \mathrm{BrN}_{3}$ 245.0522; Found 245.0535. 


\section{2-(2-Bromo-3-methylbut-2-en-1-yl)propane-1,3-diol (S18)}

Application of the general procedure described above (for S10) afforded diol S18 [780 mg, 78\% from dimethyl 2-(2-bromo-3-methylbut-2-en-1-yl)malonate ${ }^{38}$ ] as a colorless oil after purification by chromatography on silica gel, eluting with a gradient of $50 \%$ ether/petrol to pure ether. $\mathrm{R}_{f} 0.36$ (ether); $v_{\max } 3269 \mathrm{br} ; \delta_{\mathrm{H}}\left(400 \mathrm{MHz}, \mathrm{CDCl}_{3}\right) 1.62(1 \mathrm{H}, \mathrm{br} \mathrm{s}), 1.81(3 \mathrm{H}, \mathrm{s}), 1.90(3 \mathrm{H}, \mathrm{s}), 2.14-2.22(2 \mathrm{H}, \mathrm{m}), 2.60$ $(2 \mathrm{H}, \mathrm{d}, J=7.5 \mathrm{~Hz}), 3.70(2 \mathrm{H}, \mathrm{dd}, J=10.5,6.0 \mathrm{~Hz}), 3.87(2 \mathrm{H}, \mathrm{dd}, J=10.5,3.5 \mathrm{~Hz}) ; \delta_{\mathrm{C}}(100 \mathrm{MHz}$, $\left.\mathrm{CDCl}_{3}\right)$ 20.8, 25.6, 35.9, 41.2, 65.0, 119.6, 132.4; HRMS $\left(\mathrm{FI}^{+}\right) \mathrm{m} / \mathrm{z}:\left[\mathrm{M}^{+}\right]$Calcd for $\mathrm{C}_{8} \mathrm{H}_{15}{ }^{79} \mathrm{BrO}_{2}$ 222.0250; Found 222.0254.

\section{2-(Azidomethyl)-4-bromo-5-methylhex-4-en-1-ol (22g)}

Application of the general procedure described above (for S12) afforded azide 22g as a colorless oil (54 $\mathrm{mg}$, 35\% from diol $\mathbf{S 1 8}$ using 1.0 molar equivalent of $\mathrm{MsCl}$ in the first step); the diazide (22h) was also obtained as a colorless oil (31 mg, 18\%). Data for $22 \mathrm{~g}$ : $\mathrm{R}_{f} 0.28$ (petrol/ether, 2:1); $v_{\max } 3354 \mathrm{br}, 2095 \mathrm{~s}$; $\delta_{\mathrm{H}}\left(500 \mathrm{MHz}, \mathrm{CDCl}_{3}\right) 1.82(3 \mathrm{H}, \mathrm{s}), 1.91(3 \mathrm{H}, \mathrm{s}), 2.19-2.26(1 \mathrm{H}, \mathrm{m}), 2.51-2.62(2 \mathrm{H}, \mathrm{m}), 3.40(2 \mathrm{H}, \mathrm{dd}$, $J=12.0,6.0 \mathrm{~Hz}), 3.52(2 \mathrm{H}, \mathrm{dd}, J=12.0,5.0 \mathrm{~Hz}), 3.63(2 \mathrm{H}, \mathrm{dd}, J=11.0,6.0 \mathrm{~Hz}), 3.72(2 \mathrm{H}, \mathrm{dd}, J=$ 11.0, $4.0 \mathrm{~Hz}) ; \delta_{\mathrm{C}}\left(125 \mathrm{MHz}, \mathrm{CDCl}_{3}\right)$ 20.8, 25.7, 36.6, 39.8, 52.0, 62.7, 119.0, 133.0; HRMS $\left(\mathrm{ESI}^{+}\right) \mathrm{m} / z$ : $[\mathrm{M}+\mathrm{H}]^{+}$Calcd for $\mathrm{C}_{8} \mathrm{H}_{15}{ }^{79} \mathrm{BrN}_{3} \mathrm{O}$ 248.0393; Found 248.0424. Data for 22h: $\mathrm{R}_{f} 0.94$ (ether); $v_{\max } 2091 \mathrm{~s}$; $\delta_{\mathrm{H}}\left(400 \mathrm{MHz}, \mathrm{CDCl}_{3}\right) 1.82(3 \mathrm{H}, \mathrm{s}), 1.91(3 \mathrm{H}, \mathrm{s}), 2.22-2.32(1 \mathrm{H}, \mathrm{m}), 2.54(2 \mathrm{H}, \mathrm{d}, J=7.5 \mathrm{~Hz}), 3.34(2$ H, dd, $J=12.0,6.5 \mathrm{~Hz}), 3.46(2 \mathrm{H}, \mathrm{dd}, J=12.0,5.0 \mathrm{~Hz}) ; \delta_{\mathrm{C}}\left(100 \mathrm{MHz}, \mathrm{CDCl}_{3}\right) 20.8,25.7,37.1,37.7$, 51.7, 118.1, 133.6; HRMS $\left(\mathrm{FI}^{+}\right) \mathrm{m} / z$ : $\left[\mathrm{M}-\mathrm{Br}-\mathrm{N}_{2}\right]^{+}$Calcd for $\mathrm{C}_{8} \mathrm{H}_{13} \mathrm{~N}_{4}$ 165.1135; Found 165.1136 .

6-Azido-5-(azidomethyl)-3-bromo-2-methylhex-2-ene (22h) 


\begin{abstract}
Application of the general procedure described above (for S12) afforded azide $\mathbf{2 2 h}$ as a pale yellow oil (241 mg, 78\% from diol S18 using 2.2 molar equivalents of $\mathrm{MsCl}$ in the first step). Data as above.
\end{abstract}

\title{
7-Chloro-2-methylhept-2-ene (S19)
}

To an oven-dried $100 \mathrm{~mL}$ round-bottomed flask was added isopropyltriphenylphosphonium iodide (3.28 $\mathrm{g}, 7.59 \mathrm{mmol})$ and ether $(40 \mathrm{~mL})$, and the flask was cooled to $0{ }^{\circ} \mathrm{C}$ under argon. Butyllithium $(4.70 \mathrm{~mL}$, $1.60 \mathrm{M}$ in pentane, $7.52 \mathrm{mmol}$ ) was added dropwise over $1 \mathrm{~h}$, leading to an intense red coloration indicative of ylid formation. Stirring was continued for $2 \mathrm{~h}$, then a solution of 5-chloropentanal (726 mg, $6.02 \mathrm{mmol})$ in ether $(5.0 \mathrm{~mL})$ was added dropwise via syringe pump over $40 \mathrm{~min}$; the residue in the syringe was washed in with ether $(2.0 \mathrm{~mL})$, and the reaction mixture was stirred for $14 \mathrm{~h}$, during which time the ice bath thawed to $\sim \mathrm{RT}$. The cream-colored reaction mixture was quenched with brine $(70 \mathrm{~mL})$ and filtered through Celite $^{\circledR}$, washing through with ether $(60 \mathrm{~mL})$. The phases were separated and the aqueous layer was extracted with ether $(3 \times 10 \mathrm{~mL})$ and then dried $\left(\mathrm{MgSO}_{4}\right)$. The organic solution was concentrated onto silica gel and purified by eluting with petrol and the product-containing fractions collected. The combined fractions $(\sim 14 \mathrm{~mL})$ were used directly in the next reaction, although an analytical sample was obtained by careful removal of the solvent. $\mathrm{R}_{f} 0.60$ (petrol); $v_{\max } 2928 \mathrm{~s}, 1447 \mathrm{~m}$, $731 \mathrm{~s}, 653 \mathrm{~s} ; \delta_{\mathrm{H}}\left(400 \mathrm{MHz}, \mathrm{CDCl}_{3}\right) 1.48(2 \mathrm{H}$. app. quin, $J=7.5 \mathrm{~Hz}), 1.61(3 \mathrm{H}, \mathrm{s}), 1.70(3 \mathrm{H}, \mathrm{s}), 1.77(2$ $\mathrm{H}$, app. quin, $J=7.0 \mathrm{~Hz}), 2.01(2 \mathrm{H}$, app. q, $J=7.5 \mathrm{~Hz}), 3.53(2 \mathrm{H}, \mathrm{t}, J=7.0 \mathrm{~Hz}), 5.11(1 \mathrm{H}$, app. tquin, $J=7.0,1.0 \mathrm{~Hz}) ; \delta_{\mathrm{C}}\left(100 \mathrm{MHz}, \mathrm{CDCl}_{3}\right) 17.8,25.8,27.2,27.3,32.3,45.2,124.1,132.1 ; \mathrm{HRMS}\left(\mathrm{FI}^{+}\right) m / z:$ $\left[\mathrm{M}^{+}\right]$Calcd for $\mathrm{C}_{8} \mathrm{H}_{15}{ }^{35} \mathrm{Cl} 146.0857$; Found 146.0862.

\section{3-Bromo-7-chloro-2-methylhept-2-ene (S20)}

Application of the general procedure described above (for 22a) afforded bromide S20 (604 mg, 44\% overall from 5-chloropentanal) as a colorless oil. $\mathrm{R}_{f} 0.40$ (petrol); $v_{\max } 2864 \mathrm{~s}, 1447 \mathrm{~m}, 732 \mathrm{~s}, 653 \mathrm{~s} ; \delta_{\mathrm{H}}$ 
$\left(400 \mathrm{MHz} \mathrm{CDCl}_{3}\right)$ 1.64-1.82 (4 H, m) overlays $1.78(3 \mathrm{H}, \mathrm{s}), 1.87(3 \mathrm{H}, \mathrm{s}), 2.53(2 \mathrm{H}, \mathrm{t}, J=7.0 \mathrm{~Hz})$, $3.56(2 \mathrm{H}, \mathrm{t}, J=6.5 \mathrm{~Hz}) ; \delta_{\mathrm{C}}\left(100 \mathrm{MHz}, \mathrm{CDCl}_{3}\right) 20.6,25.5,25.8,31.6,36.8,45.0,121.2$, 130.9; HRMS $\left(\mathrm{FI}^{+}\right) \mathrm{m} / z:\left[\mathrm{M}^{+}\right]$Calcd for $\mathrm{C}_{8} \mathrm{H}_{14}{ }^{79} \mathrm{Br}^{35} \mathrm{Cl} 223.9962$; Found 223.9969 .

\section{7-Azido-3-bromo-2-methylhept-2-ene (22i)}

To an oven-dried $25 \mathrm{~mL}$ round-bottomed flask was added chloride $\mathbf{S 2 0}$ (600 mg, 2.66 mmol), NaN 3 (346 $\mathrm{mg}, 5.32 \mathrm{mmol})$ and DMSO $(4.0 \mathrm{~mL})$, and the reaction mixture was stirred at $50{ }^{\circ} \mathrm{C}$ under argon for 24 h. The mixture was diluted with petrol $(20 \mathrm{~mL})$ and washed with water $(3 \times 5 \mathrm{~mL})$. The combined aqueous washes were back-extracted with petrol $(3 \times 20 \mathrm{~mL})$ and the combined organic portions were concentrated onto silica gel and purified by eluting with petrol to yield a colorless oil (528 mg, $86 \%$ ). $\mathrm{R}_{f}$ 0.43 (petrol); $v_{\max } 2092 \mathrm{~s} ; \delta_{\mathrm{H}}\left(400 \mathrm{MHz}, \mathrm{CDCl}_{3}\right) 1.57-1.66(4 \mathrm{H}, \mathrm{m}), 1.78(3 \mathrm{H}, \mathrm{s}), 1.88(3 \mathrm{H}, \mathrm{s}), 2.53(2$ $\mathrm{H}, \mathrm{t}, J=6.5 \mathrm{~Hz}), 3.30(2 \mathrm{H}, \mathrm{t}, J=6.5 \mathrm{~Hz}) ; \delta_{\mathrm{C}}\left(100 \mathrm{MHz}, \mathrm{CDCl}_{3}\right) 20.6,25.5,25.6,28.0,37.0,51.5,121.2$, 130.9; HRMS $\left(\mathrm{FI}^{+}\right) m / z:\left[\mathrm{M}-\mathrm{Br}-\mathrm{N}_{2}\right]^{+}$Calcd for $\mathrm{C}_{8} \mathrm{H}_{14} \mathrm{~N}$ 124.1121; Found 124.1062.

\section{5-(Prop-1-en-2-yl)-3,4-dihydro-2H-pyrrole (23a); general procedure for the thermolysis of azidoalkyl vinyl bromides 22}

To an oven-dried $14 \mathrm{~mL}$ Ace pressure tube was added an $\sim 0.05 \mathrm{M}$ solution of azide 22a $(60 \mathrm{mg}, 0.28$ mmol) in toluene $(5.5 \mathrm{~mL})$, solid $\mathrm{K}_{2} \mathrm{CO}_{3}(381 \mathrm{mg}, 2.76 \mathrm{mmol})$ and $\mathrm{NaOH}(20 \mathrm{mg}, 0.50 \mathrm{mmol})$. The reaction vessel was flushed with argon, and the thread was sealed with PTFE tape and the PTFE screwcap fitted with FETFE $^{\circledR}$ O-ring. The whole tube was wrapped in cotton wool/aluminum foil, and the mixture was stirred at $150{ }^{\circ} \mathrm{C}$ for $44 \mathrm{~h}$. Stirring was maintained vigorously to disperse the inorganic solids evenly throughout the solution. The vessel was removed from the oil bath, cooled to RT, and the solution was filtered through a sintered glass funnel, washing through with ether $(20 \mathrm{~mL})$, and concentrated to a toluene solution that was purified on silica gel, eluting with $0.1 \%$ triethylamine/petrol to $0.1 \%$ 
triethylamine $/ 10 \%$ ether/petrol. The volatile product was isolated as a yellow oil $(22.5 \mathrm{mg}, 73 \%)$. $\mathrm{R}_{f} 0.33$ (ether); $v_{\max } 1633 \mathrm{~s} ; \delta_{\mathrm{H}}\left(500 \mathrm{MHz}, \mathrm{C}_{6} \mathrm{D}_{6}\right) 1.45(2 \mathrm{H}$, app. quin, $J=7.5 \mathrm{~Hz}), 2.20(3 \mathrm{H}$, app. s), $2.26(2 \mathrm{H}$, tt, $J=8.0,2.0 \mathrm{~Hz}), 3.85(2 \mathrm{H}$, app. t, $J=7.5 \mathrm{~Hz}), 5.16\left(1 \mathrm{H}\right.$, app. s), $5.23(1 \mathrm{H}, \mathrm{s}) ; \delta_{\mathrm{C}}\left(125 \mathrm{MHz}, \mathrm{C}_{6} \mathrm{D}_{6}\right)$ 19.7, 23.1, 34.0, 61.9, 119.6, 142.2, 173.9; HRMS $\left(\mathrm{ESI}^{+}\right) \mathrm{m} / z:[\mathrm{M}+\mathrm{H}]^{+}$Calcd for $\mathrm{C}_{7} \mathrm{H}_{12} \mathrm{~N}$ 110.0964; Found 110.0963.

\section{5-Cyclopentenyl-3,4-dihydro-2H-pyrrole (23c)}

Application of the general procedure described above (for 23a) afforded cyclic imine $\mathbf{2 3 c}(43 \mathrm{mg}, 78 \%$ from azide 22c) as a pale yellow oil. $\mathrm{R}_{f} 0.34$ (1\% MeOH in ether); $v_{\max } 1680 \mathrm{~m}, 1629 \mathrm{~m}, 1590 \mathrm{~m}, 1260 \mathrm{~s}$; $\delta_{\mathrm{H}}\left(500 \mathrm{MHz}, \mathrm{CDCl}_{3}\right) 1.90(2 \mathrm{H}$, app. quin, $J=7.5 \mathrm{~Hz}), 1.96(2 \mathrm{H}$, app. quin, $J=7.5 \mathrm{~Hz}), 2.49-2.53(2$ H, m), 2.65-2.72 (4 H, m), $3.94(2 \mathrm{H}, \mathrm{t}, J=7.0 \mathrm{~Hz}), 6.19(1 \mathrm{H}$, app. t, $J=2.0 \mathrm{~Hz}) ; \delta_{\mathrm{C}}\left(125 \mathrm{MHz}, \mathrm{CDCl}_{3}\right)$ 22.8, 23.4, 32.1, 33.7, 35.1, 61.4, 137.5, 141.1, 171.6; HRMS $\left(\mathrm{ESI}^{+}\right) \mathrm{m} / z$ : $[\mathrm{M}+\mathrm{H}]^{+}$Calcd for $\mathrm{C}_{9} \mathrm{H}_{14} \mathrm{~N}$ 136.1121; Found 136.1116.

\section{5-Cyclohexenyl-3,4-dihydro-2H-pyrrole (23d $)^{44}$}

Application of the general procedure described above (for 23a) afforded cyclic imine $23 \mathbf{d}$ (73 $\mathrm{mg}, 84 \%$ from azide $22 \mathrm{~d}$ ) as a pale yellow oil. $\mathrm{R}_{f} 0.40$ (ether); $v_{\max } 1642 \mathrm{~m} ; \delta_{\mathrm{H}}\left(400 \mathrm{MHz}, \mathrm{CDCl}_{3}\right) 1.61-1.71(4 \mathrm{H}$, m), $1.86(2 \mathrm{H}$, app. quin, $J=7.5 \mathrm{~Hz}), 2.16-2.21(2 \mathrm{H}, \mathrm{m}), 2.36-2.42(2 \mathrm{H}, \mathrm{m}), 2.65(2 \mathrm{H}, \mathrm{tt}, J=8.0,1.5$ $\mathrm{Hz}), 3.91(2 \mathrm{H}, \mathrm{t}, J=6.5 \mathrm{~Hz}), 6.26(1 \mathrm{H}, \mathrm{m}) ; \delta_{\mathrm{C}}\left(100 \mathrm{MHz}, \mathrm{CDCl}_{3}\right) 22.2,22.5,22.6,25.1,26.1,33.9$, 61.2, 134.6, 135.0, 175.2; HRMS (FI') m/z: [M+] Calcd for $\mathrm{C}_{10} \mathrm{H}_{15} \mathrm{~N}$ 149.1199; Found 149.1199.

\section{3,3-Dimethyl-5-(prop-1-en-2-yl)-3,4-dihydro-2H-pyrrole (23f)}

Application of the general procedure described above (for 23a) afforded cyclic imine $23 \mathbf{f}$ (15 mg, 71\% from azide $22 \mathrm{f}$ ) as a pale yellow oil. $\mathrm{R}_{f} 0.46$ (petrol/ether, $\left.1: 1\right)$; $v_{\max } 1591 \mathrm{~s} ; \delta_{\mathrm{H}}\left(500 \mathrm{MHz}, \mathrm{C}_{6} \mathrm{D}_{6}\right) 0.84(6$ 


\begin{abstract}
H, s), $2.20\left(5 \mathrm{H}\right.$, app. s), $3.66(2 \mathrm{H}, \mathrm{s}), 5.16(1 \mathrm{H}, \mathrm{s}), 5.24(1 \mathrm{H}, \mathrm{s}) ; \delta_{\mathrm{C}}\left(125 \mathrm{MHz}, \mathrm{C}_{6} \mathrm{D}_{6}\right)$ 19.4, 27.9, 38.4, 49.2, 75.2, 119.6, 142.4, 173.6; HRMS (ESI $\left.{ }^{+}\right) \mathrm{m} / z$ : $[\mathrm{M}+\mathrm{H}]^{+}$Calcd for $\mathrm{C}_{9} \mathrm{H}_{16} \mathrm{~N}$ 138.1277; Found 138.1275.
\end{abstract}

\title{
[(5-(Prop-1-en-2-yl)-3,4-dihydro-2H-pyrrol-3-yl]methanol (23g)
}

Application of the general procedure described above (for 23a) afforded cyclic imine $\mathbf{2 3 g}$ ( $46 \mathrm{mg}, 82 \%$ from azide $22 \mathrm{~g}$ ) as a pale yellow oil. $\mathrm{R}_{f} 0.17$ (ether); $v_{\max } 3272 \mathrm{br}, 1596 \mathrm{~m} ; \delta_{\mathrm{H}}\left(500 \mathrm{MHz}, \mathrm{CDCl}_{3}\right) 2.00(3$ $\mathrm{H}, \mathrm{s}), 2.54-2.63(2 \mathrm{H}, \mathrm{m}), 2.81-2.87(2 \mathrm{H}, \mathrm{m}), 3.49$ and $3.53(2 \times 1 \mathrm{H}, 2 \times \mathrm{dd}, J=10.5,6.5 \mathrm{~Hz}), 3.78(1$ H, dd, $J=16.5,4.0 \mathrm{~Hz}), 4.04(1 \mathrm{H}$, app. dd, $J=16.5,7.0 \mathrm{~Hz}), 5.42$ and $5.46(2 \times 1 \mathrm{H}, 2 \times \mathrm{s}) ; \delta_{\mathrm{C}}(125$ $\left.\mathrm{MHz}, \mathrm{CDCl}_{3}\right)$ 19.3, 37.6, 39.2, 64.0, 65.4, 121.5, 140.7, 174.7; HRMS $\left(\mathrm{ESI}^{+}\right) \mathrm{m} / z:[\mathrm{M}+\mathrm{H}]^{+} \mathrm{Calcd}$ for $\mathrm{C}_{8} \mathrm{H}_{14} \mathrm{NO}$ 140.1070; Found 140.1065.

\section{3-(Azidomethyl)-5-(prop-1-en-2-yl)-3,4-dihydro-2H-pyrrole (23h)}

Application of the general procedure described above (for 23a) afforded cyclic imine $\mathbf{2 3 h}$ (32 $\mathrm{mg}, 76 \%$ from azide $22 \mathrm{~h}$ ) as a pale yellow oil. $\mathrm{R}_{f} 0.65$ (ether); $v_{\max } 2095 \mathrm{~s}$; $\delta_{\mathrm{H}}\left(400 \mathrm{MHz}, \mathrm{CDCl}_{3}\right) 2.02(3 \mathrm{H}, \mathrm{s})$, 2.54-2.68 (2 H, m), $2.91(1 \mathrm{H}$, app. qt, $J=7.5,1.5 \mathrm{~Hz}), 3.24(1 \mathrm{H}, \mathrm{dd}, J=12.0,7.5 \mathrm{~Hz}), 3.32(1 \mathrm{H}, \mathrm{dd}, J$ $=12.0,6.5 \mathrm{~Hz}), 3.76(1 \mathrm{H}, \mathrm{dd}, J=17.0,4.5 \mathrm{~Hz}), 4.11(1 \mathrm{H}, \mathrm{dd}, J=17.0,8.0 \mathrm{~Hz}), 5.42$ and $5.49(2 \times 1$ $\mathrm{H}, 2 \times \mathrm{s}) ; \delta_{\mathrm{C}}\left(100 \mathrm{MHz}, \mathrm{CDCl}_{3}\right)$ 19.3, 36.9, 38.5, 55.1, 64.8, 121.5, 140.7, 174.1; HRMS $\left(\mathrm{ESI}^{+}\right) \mathrm{m} / z$ : $[\mathrm{M}+\mathrm{H}]^{+}$Calcd for $\mathrm{C}_{8} \mathrm{H}_{13} \mathrm{~N}_{4}$ 165.1135; Found 165.1134.

\section{6-(Prop-1-en-2-yl)-2,3,4,5-tetrahydropyridine (23i) $)^{22}$}

Application of the general procedure described above (for 23a) afforded cyclic imine $\mathbf{2 3 i}$ ( $17 \mathrm{mg}, 32 \%$ from azide $22 \mathrm{~h}$ ) as a colorless oil. $\mathrm{R}_{f} 0.10$ (ether); $v_{\max } 2096 \mathrm{~m}, 1767 \mathrm{~s}, 1725 \mathrm{~s}, 1695 \mathrm{~s}, 1619 \mathrm{~s} ; \delta_{\mathrm{H}}(500$ MHz, $\left.\mathrm{C}_{6} \mathrm{D}_{6}\right) 1.17-1.22(2 \mathrm{H}, \mathrm{m}), 1.26-1.31(2 \mathrm{H}, \mathrm{m}), 2.02(2 \mathrm{H}, \mathrm{tt}, J=6.5,2.0 \mathrm{~Hz}), 2.17(3 \mathrm{H}, \mathrm{s}), 3.70$ 


\begin{abstract}
(2 H, app. t, $J=5.5 \mathrm{~Hz}), 5.23$ and $5.25(2 \times 1 \mathrm{H}, 2 \times \mathrm{s}) ; \delta_{\mathrm{C}}\left(125 \mathrm{MHz}, \mathrm{C}_{6} \mathrm{D}_{6}\right) 20.0$ (two peaks), 22.2, 25.3, 50.0, 115.4, 146.8, 165.3; HRMS (ESI $\left.{ }^{+}\right) m / z:[\mathrm{M}+\mathrm{H}]^{+}$Calcd for $\mathrm{C}_{8} \mathrm{H}_{14} \mathrm{~N}$ 124.1121; Found 124.1119.
\end{abstract}

\title{
Dimethyl 2-(pyrrolidin-2-ylidene)malonate $(24 e)^{18}$ and methyl 2-oxo-4,5,6,6a-tetrahydro-2H- furo[3,2-b]pyrrole-3-carboxylate (25e)
}

Application of the general procedure described above (for 23a) using azide 22e, with heating at $100{ }^{\circ} \mathrm{C}$ for $11 \mathrm{~h}$, led to the formation of a white solid while the solution became yellow. The pressure tube was removed from the oil bath, cooled to $\mathrm{RT}$, and the reaction mixture was filtered to collect the solid precipitate which was dried at $\sim 1 \mathrm{mmHg}$ and identified as the bicyclic product $25 \mathrm{e}(24 \mathrm{mg}, 60 \%)$. Meanwhile, the filtrate was concentrated and purified on silica gel, eluting with a gradient of petrol to $25 \%$ ether/petrol, yielding the enamide $24 \mathrm{e}\left(9 \mathrm{mg}, 20 \%\right.$ ) as a red oil. Data for $24 \mathrm{e}: \mathrm{R}_{f} 0.34$ (ether); $v_{\max }$ 3308br, 1642s, 1573s; $\delta_{\mathrm{H}}\left(500 \mathrm{MHz}, \mathrm{CDCl}_{3}\right) 2.05(2 \mathrm{H}$, app. quin, $J=7.5 \mathrm{~Hz}), 3.13(2 \mathrm{H}, \mathrm{t}, J=8.0 \mathrm{~Hz})$, $3.62(2 \mathrm{H}, \mathrm{t}, J=7.5 \mathrm{~Hz}), 3.73(3 \mathrm{H}, \mathrm{s}), 3.75(3 \mathrm{H}, \mathrm{s}), 9.66(1 \mathrm{H}, \mathrm{br}) ; \delta_{\mathrm{C}}\left(125 \mathrm{MHz}, \mathrm{CDCl}_{3}\right) 21.8,34.6$, 47.7, 51.1, 51.3, 86.8, 168.2, 170.4, 174.1; m/z (ESI+) 222.1 $\left(\mathrm{MNa}^{+}\right), 200.1\left(\mathrm{MH}^{+}\right)$. Data for 25e: M.p $235^{\circ} \mathrm{C} ; \mathrm{R}_{f} 0.13$ (ether/methanol, 8:1); $v_{\max } 3165 \mathrm{br}, 1739 \mathrm{~m}, 1702 \mathrm{~m}, 1636 \mathrm{~s} ; \delta_{\mathrm{H}}\left(400 \mathrm{MHz}, \mathrm{CDCl}_{3}\right) 2.13$ (1 H, tddd, $J=11.5,11.0,8.5 \mathrm{~Hz}), 2.61(1 \mathrm{H}, \mathrm{ddd}, J=11.5,7.0,4.5 \mathrm{~Hz}), 3.83(3 \mathrm{H}, \mathrm{s}), 3.86-4.00(2 \mathrm{H}$, m), $5.00(1 \mathrm{H}, \mathrm{dd}, J=12.0,7.0 \mathrm{~Hz}), 6.86(1 \mathrm{H}, \mathrm{br}) ; \delta_{\mathrm{C}}\left(125 \mathrm{MHz}, \mathrm{CDCl}_{3}\right) 32.5,50.7,51.7,77.6,85.9$, 164.0, 170.8, 180.8; HRMS (ESI ${ }^{+}$) m/z: $[\mathrm{M}+\mathrm{Na}]^{+}$Calcd for $\mathrm{C}_{8} \mathrm{H}_{9} \mathrm{NNaO}_{4}$ 206.0424; Found 206.0429.

\section{5-(1-Bromocyclobutyl)-3,4-dihydro-2H-pyrrole (27), 5-[1-(bromomethyl)cyclopropyl]-3,4-dihydro- 2H-pyrrole (29), [1-(3,4-dihydro-2H-pyrrol-5-yl)cyclopropyl]methanol (30), and 5,5'-(4- vinylcyclohex-1-ene-1,4-diyl)bis(3,4-dihydro-2H-pyrrole) (32)}

Application of the general procedure described above (for 23a) using azide 22b gave product mixtures containing variable quantities of secondary products $\mathbf{2 7}, \mathbf{2 9}, \mathbf{3 0}$, and $\mathbf{3 2}$, each of which was typically 
isolated in less than $10 \%$ yield. Data for $27: \mathrm{R}_{f} 0.78$ (ether); $v_{\max } 1627 \mathrm{~m} ; \delta_{\mathrm{H}}\left(500 \mathrm{MHz}, \mathrm{CDCl}_{3}\right) 1.86(1$ $\mathrm{H}, \mathrm{dtt}, J=10.5,8.5,6.5 \mathrm{~Hz}), 1.99(2 \mathrm{H}$, app. quin, $J=7.5 \mathrm{~Hz}), 2.23-2.32(1 \mathrm{H}, \mathrm{m}), 2.58-2.74(2 \mathrm{H}, \mathrm{m})$, 2.74-2.79 (2 H, m), 2.89-2.96 (2 H, m), $3.93(2 \mathrm{H}, \mathrm{tt}, J=7.0,2.0 \mathrm{~Hz}) ; \delta_{\mathrm{C}}\left(125 \mathrm{MHz}, \mathrm{CDCl}_{3}\right) 17.1,23.4$, 33.3, 37.9 (two peaks), 61.1, 178.2; $\mathrm{HRMS}\left(\mathrm{ESI}^{+}\right) \mathrm{m} / z$ : $[\mathrm{M}+\mathrm{H}]^{+}$Calcd for $\mathrm{C}_{8} \mathrm{H}_{13}{ }^{79} \mathrm{BrN}$ 202.0226; Found 202.0224. Data for 29: $\mathrm{R}_{f} 0.55$ (ether); $v_{\max } 1629 \mathrm{~m}$; $\delta_{\mathrm{H}}\left(500 \mathrm{MHz}, \mathrm{C}_{6} \mathrm{D}_{6}\right) 0.53(2 \mathrm{H}, \mathrm{dt}, J=7.0,4.5 \mathrm{~Hz})$, $1.09(2 \mathrm{H}, \mathrm{dt}, J=7.0,4.5 \mathrm{~Hz}), 1.40(2 \mathrm{H}$, app. quin. $J=7.5 \mathrm{~Hz}), 1.95(2 \mathrm{H}, \mathrm{tt}, J=8.0,2.0 \mathrm{~Hz}), 3.36(2$ $\mathrm{H}, \mathrm{s}), 3.71(2 \mathrm{H}, \mathrm{tt}, J=7.0,2.0 \mathrm{~Hz}) ; \delta_{\mathrm{C}}\left(125 \mathrm{MHz}, \mathrm{C}_{6} \mathrm{D}_{6}\right) 17.8,23.1,26.2,34.0,40.5,61.2,175.7$; HRMS $\left(\mathrm{ESI}^{+}\right) \mathrm{m} / z:[\mathrm{M}+\mathrm{H}]^{+}$Calcd for $\mathrm{C}_{8} \mathrm{H}_{13}{ }^{79} \mathrm{BrN}$ 202.0226; Found 202.0228. Data for 30: $\mathrm{R}_{f} 0.18(5 \%$ methanol/ether); $v_{\max } 3334 \mathrm{br}, 1621 \mathrm{~m} ; \delta_{\mathrm{H}}\left(500 \mathrm{MHz}, \mathrm{CDCl}_{3}\right) 0.84(2 \mathrm{H}, \mathrm{dt}, J=6.5,4.5 \mathrm{~Hz}), 0.99(2 \mathrm{H}$, dt, $J=6.5,4.5 \mathrm{~Hz}), 1.83(2 \mathrm{H}$, app. quin, $J=7.0 \mathrm{~Hz}), 2.23(2 \mathrm{H}, \mathrm{tt}, J=7.5,1.5 \mathrm{~Hz}), 3.65(2 \mathrm{H}, \mathrm{s}), 3.85$ $(2 \mathrm{H}, \mathrm{tt}, J=7.0,1.5 \mathrm{~Hz}) ; \delta_{\mathrm{C}}\left(125 \mathrm{MHz}, \mathrm{CDCl}_{3}\right) 11.8,22.1,26.2,33.3,61.0,68.6,180.9$; HRMS $\left(\mathrm{ESI}^{+}\right)$ $m / z$ : $[\mathrm{M}+\mathrm{Na}]^{+}$Calcd for $\mathrm{C}_{8} \mathrm{H}_{13} \mathrm{NNaO}$ 162.0889; Found 162.0889. Data for 32 (only observed as a contaminant of incompletely-separated alcohol 30): $\mathrm{R}_{f} 0.28$ (methanol/ether, 1:10); $\delta_{\mathrm{H}}\left(500 \mathrm{MHz}, \mathrm{C}_{6} \mathrm{D}_{6}\right)$ 1.39-1.46 (4 H, m), 1.78 (1 H, ddd, $J=13.5,8.0,5.5 \mathrm{~Hz}), 1.97$ (1 H, ddd, $J=13.0,6.5,6.5 \mathrm{~Hz}), 2.16(2$ $\mathrm{H}, \mathrm{tq}, J=8.0,2.0 \mathrm{~Hz}), 2.27(2 \mathrm{H}, \mathrm{tt}, J=8.0,2.0 \mathrm{~Hz})$ overlays $2.25-2.28(1 \mathrm{H}, \mathrm{m}), 2.60-2.69(1 \mathrm{H}, \mathrm{m})$, 2.81-2.87 (1 H, m), $3.02(1 \mathrm{H}$, br d, $J=18.5 \mathrm{~Hz}), 3.68(2 \mathrm{H}, \mathrm{tq}, J=7.0,1.5 \mathrm{~Hz}), 3.86(2 \mathrm{H}$, app. t, $J=$ $7.0 \mathrm{~Hz}), 4.95-5.00(2 \mathrm{H}, \mathrm{m}), 5.74(1 \mathrm{H}, \mathrm{dd}, J=17.5,10.5 \mathrm{~Hz}), 6.03(1 \mathrm{H}, \mathrm{t}, J=4.0 \mathrm{~Hz}) ; \delta_{\mathrm{C}}(125 \mathrm{MHz}$, $\left.\mathrm{C}_{6} \mathrm{D}_{6}\right) 22.8,23.1,23.2,30.4,33.2,33.8,34.1,44.8,61.0,61.5,113.7,1131.8,134.8,143.2,173.4,178.9$; HRMS (ESI ${ }^{+}$) m/z: $[\mathrm{M}+\mathrm{Na}]^{+}$Calcd for $\mathrm{C}_{16} \mathrm{H}_{22} \mathrm{~N}_{2} \mathrm{Na}$ 265.1675; Found 265.1667.

\section{8-Methyl-6,6-diphenyl-2,3,6,7-tetrahydroindolizin-5(1H)-one (37a); general procedure for the thermolysis of azidoalkyl vinyl bromides 22, and one-pot formal cycloaddition with diphenylketene}

The general procedure described above (for 23a) was followed with azide 22a (100 $\mathrm{mg}, 0.459 \mathrm{mmol}$ ) until the point where the heated vessel is removed from the oil bath and cooled to RT. The PTFE screw 
cap was removed and replaced with a suba cap, the headspace was flushed with argon, and a solution of diphenylketene $(266 \mathrm{mg}, 1.37 \mathrm{mmol})$ in toluene $(2.0 \mathrm{~mL})$ was added dropwise over $50 \mathrm{~min}$, causing the yellow solution to become bright yellow. After stirring for $15 \mathrm{~h}$, the cloudy solution was filtered through a sintered glass funnel, washing through with ether $(30 \mathrm{~mL})$; the filtrate was concentrated onto silica, and the residue was purified on silica gel, eluting with a gradient of $0.5 \%$ triethylamine/petrol to $0.5 \%$ triethylamine $/ 25 \%$ ether/petrol. The lactam product was isolated as a white crystalline solid (112 mg, 80\%). M.p $156-160{ }^{\circ} \mathrm{C} ; \mathrm{R}_{f} 0.28$ (petrol/ether, 1:1); $v_{\max } 2964 \mathrm{w}, 1651 \mathrm{~s}, 1395 \mathrm{~m}, 1260 \mathrm{~m}, 1021 \mathrm{~m}, 800 \mathrm{~m}$, 728s; $\delta_{\mathrm{H}}\left(400 \mathrm{MHz}, \mathrm{C}_{6} \mathrm{D}_{6}\right) 1.11(2 \mathrm{H}$, app. quin, $J=7.0 \mathrm{~Hz}), 1.49(3 \mathrm{H}$, app. quin, $J=1.0 \mathrm{~Hz}), 1.80(2$ H, ddd, $J=7.5,3.5,2.0 \mathrm{~Hz}), 2.67(2 \mathrm{H}$, oct, $J=1.0 \mathrm{~Hz}), 3.52(2 \mathrm{H}$, app. t, $J=7.0 \mathrm{~Hz}), 7.05(2 \mathrm{H}, \mathrm{tt}, J=$ 7.5, 1.0 Hz), 7.13-7.18 (4 H, m), 7.38-7.41 (4 H, m); $\delta_{\mathrm{C}}\left(100 \mathrm{MHz}, \mathrm{C}_{6} \mathrm{D}_{6}\right)$ 17.5, 21.9, 27.1, 42.7, 46.5, 57.3, 103.4, 126.9, 128.2, 129.1, 133.7, 144.1, 169.2; HRMS $\left(\mathrm{ESI}^{+}\right) \mathrm{m} / z:[\mathrm{M}+\mathrm{Na}]^{+}$Calcd for $\mathrm{C}_{21} \mathrm{H}_{21} \mathrm{NNaO} 326.1515$; Found 326.1500 .

\section{6,6-Diphenyl-2,3,6a,7,8,9-hexahydro-1H-cyclopentalg/indolizin-5(6H)-one (37c)}

Application of the general procedure described above (for 37a) afforded lactam 37c (52 mg, 77\% from azide 22c) as a pale orange amorphous solid. M.p $159-161^{\circ} \mathrm{C} ; \mathrm{R}_{f} 0.20$ (petrol/ether, $1: 1$ ); $v_{\max } 1645 \mathrm{~s} ; \delta_{\mathrm{H}}$ (500 MHz, $\left.\mathrm{C}_{6} \mathrm{D}_{6}\right) 1.21-1.36(3 \mathrm{H}, \mathrm{m}), 1.50-1.59(2 \mathrm{H}, \mathrm{m}), 1.75(1 \mathrm{H}$, app. dq, $J=11.5,6.5 \mathrm{~Hz}), 1.97-$ $2.01(2 \mathrm{H}, \mathrm{m}), 2.02-2.09(1 \mathrm{H}, \mathrm{m}), 2.16(1 \mathrm{H}, \mathrm{dd}, J=16.0,8.0 \mathrm{~Hz}), 3.37-3.42(1 \mathrm{H}, \mathrm{m}), 3.45-3.56(2 \mathrm{H}$, m), $7.03(1 \mathrm{H}$, app. t, $J=7.0 \mathrm{~Hz}), 7.10(2 \mathrm{H}$, app. t, $J=7.5 \mathrm{~Hz}), 7.13(1 \mathrm{H}$, app. t, $J=7.0 \mathrm{~Hz}), 7.22(2 \mathrm{H}$, app. t, $J=7.5 \mathrm{~Hz}), 7.35(2 \mathrm{H}$, app. d, $J=8.0 \mathrm{~Hz}), 7.52(2 \mathrm{H}$, app. d, $J=8.0 \mathrm{~Hz}) ; \delta_{\mathrm{C}}\left(125 \mathrm{MHz}, \mathrm{C}_{6} \mathrm{D}_{6}\right)$ $22.5,26.0,27.4,28.1,28.4,46.5,50.4,60.1,114.3,126.5,127.1,127.8,128.4,129.5,130.5,130.8,140.5$, 145.5, 169.7; HRMS $\left(\mathrm{ESI}^{+}\right) \mathrm{m} / z$ : $[\mathrm{M}+\mathrm{Na}]^{+}$Calcd for $\mathrm{C}_{23} \mathrm{H}_{23} \mathrm{NNaO}$ 352.1672; Found 352.1673.

6,6-Diphenyl-2,3,6,6a,7,8,9,10-octahydropyrrolo[2,1-a]isoquinolin-5(1H)-one (37d) 
Application of the general procedure described above (for 37a) afforded lactam $\mathbf{3 7 d}$ (54 mg, $80 \%$ from azide 22d) as a low-density white solid foam. M.p 51-53 ${ }^{\circ} \mathrm{C} ; \mathrm{R}_{f} 0.28$ (petrol/ether, 1:1); $v_{\max } 1651 \mathrm{~s} ; \delta_{\mathrm{H}}$ (400 MHz, C6 6 6) 0.92-1.18 (6 H, m), 1.51-1.54 (1 H, m), 1.60-1.63 (1 H, m), 1.87-2.00 (3 H, m), 2.19$2.23(1 \mathrm{H}, \mathrm{m}), 2.68$ (1 H, app. d, $J=11.5 \mathrm{~Hz}), 3.34(1 \mathrm{H}, \mathrm{ddd}, J=11.5,8.0,5.5 \mathrm{~Hz}), 3.52(1 \mathrm{H}, \mathrm{ddd}, J=$ 11.5, 7.5, 7.0 Hz), 7.05 (2 H, tq, $J=7.5,1.5 \mathrm{~Hz}), 7.13-7.18(4 \mathrm{H}, \mathrm{m}), 7.46(2 \mathrm{H}$, app. d, $J=7.5 \mathrm{~Hz}), 7.75$ (2 H, app. d, $J=7.5 \mathrm{~Hz}) ; \delta_{\mathrm{C}}\left(100 \mathrm{MHz}, \mathrm{C}_{6} \mathrm{D}_{6}\right) 21.5,27.1,27.4,29.2,30.9,31.7,46.7,49.6,59.8,112.6$, 126.3, 127.4, 127.6, 129.3, 129.6, 131.0, 143.8, 144.6, 168.1 [one Ph resonance obscured]; HRMS (ESI $\left.{ }^{+}\right)$ $m / z:[\mathrm{M}+\mathrm{Na}]^{+}$Calcd for $\mathrm{C}_{24} \mathrm{H}_{25} \mathrm{NNaO} 366.1828$; Found 366.1812.

\section{2,2,8-Trimethyl-6,6-diphenyl-2,3,6,7-tetrahydroindolizin-5(1H)-one (37f)}

Application of the general procedure described above (for 37a) afforded lactam $\mathbf{3 7 f}$ (63 mg, 91\% from azide 22f) as a yellow viscous oil. $\mathrm{R}_{f} 0.60$ (petrol/ether, 1:1); $v_{\max } 2957 \mathrm{w}, 1658 \mathrm{~s}, 1397 \mathrm{~m}, 1035 \mathrm{w}, 698 \mathrm{~s}$; $\delta_{\mathrm{H}}\left(400 \mathrm{MHz}, \mathrm{C}_{6} \mathrm{D}_{6}\right) 0.57(6 \mathrm{H}, \mathrm{s}), 1.52(3 \mathrm{H}, \mathrm{s}), 1.71(2 \mathrm{H}, \mathrm{s}), 2.70(2 \mathrm{H}, \mathrm{s}), 3.35(2 \mathrm{H}, \mathrm{s}), 7.06(2 \mathrm{H}, \mathrm{tt}$, $J=7.5,1.0 \mathrm{~Hz}), 7.14-7.18(4 \mathrm{H}, \mathrm{m}), 7.40-7.42(4 \mathrm{H}, \mathrm{m}) ; \delta_{\mathrm{C}}\left(100 \mathrm{MHz}, \mathrm{C}_{6} \mathrm{D}_{6}\right)$ 17.5, 26.0, 35.8, 41.8, 42.7, 57.1, 58.8, 104.5, 126.9, 128.2, 129.1, 133.9, 143.9, 169.4; HRMS $\left(\mathrm{ESI}^{+}\right) \mathrm{m} / z:[\mathrm{M}+\mathrm{Na}]^{+}$Calcd for $\mathrm{C}_{23} \mathrm{H}_{25} \mathrm{NNaO}$ 354.1828; Found 354.1821.

\section{(8-Methyl-5-oxo-6,6-diphenyl-1,2,3,5,6,7-hexahydroindolizin-2-yl)methyl 2,2-diphenylacetate (37g)}

Application of the general procedure described above (for 37a) afforded lactam $\mathbf{3 7 g}$ (60 mg, 74\% from azide $22 \mathrm{~g}$ ) as a yellow oil. $\mathrm{R}_{f} 0.40$ (petrol/ether, $\left.1: 1\right) ; v_{\max } 1735 \mathrm{~s}, 1655 \mathrm{~m} ; \delta_{\mathrm{H}}\left(500 \mathrm{MHz}, \mathrm{CDCl}_{3}\right) 1.70(3$ H, s), $2.12(1 \mathrm{H}, \mathrm{d}, J=11.0 \mathrm{~Hz}), 2.45-2.55(2 \mathrm{H}, \mathrm{m}), 2.88(2 \mathrm{H}, \mathrm{s}), 3.49(1 \mathrm{H}, \mathrm{dd}, J=12.0,5.5 \mathrm{~Hz}), 3.79$ $(1 \mathrm{H}, \mathrm{dd}, J=12.0,7.0 \mathrm{~Hz}), 3.90(1 \mathrm{H}, \mathrm{dd}, J=11.0,7.5 \mathrm{~Hz}), 3.99(1 \mathrm{H}, \mathrm{dd}, J=11.0,6.5 \mathrm{~Hz}), 5.01(1 \mathrm{H}$, s), 7.11-7.35 (20 H, m); $\delta_{\mathrm{C}}\left(125 \mathrm{MHz}, \mathrm{CDCl}_{3}\right)$ 17.7, 30.1, 34.6, 42.1, 48.7, 56.9, 57.1, 66.0, 106.0, 126.9, 
127.0, 127.5, 128.1, 128.2, 128.4, 128.5, 128.7, 128.8, 131.8, 138.5, 142.5, 142.9, 169.9, 172.4 [some Ph resonances obscured]; HRMS (ESI ${ }^{+}$) m/z: $[\mathrm{M}+\mathrm{Na}]^{+}$Calcd for $\mathrm{C}_{36} \mathrm{H}_{33} \mathrm{NNaO}_{3}$ 550.2353; Found 550.2334.

\section{2-(Azidomethyl)-8-methyl-6,6-diphenyl-2,3,6,7-tetrahydroindolizin-5(1H)-one (37h)}

Application of the general procedure described above (for 37a) afforded lactam $\mathbf{3 7 h}$ (38 $\mathrm{mg}, 58 \%$ from azide $22 \mathrm{~h}$ ) as a yellow oil. $\mathrm{R}_{f} 0.34$ (petrol/ether, $\left.1: 1\right)$; $v_{\max } 2097 \mathrm{~s}, 1655 \mathrm{~m} ; \delta_{\mathrm{H}}\left(400 \mathrm{MHz}, \mathrm{CDCl}_{3}\right) 1.76(3$ $\mathrm{H}, \mathrm{s}), 2.27(1 \mathrm{H}, \mathrm{dd}, J=15.5,4.0 \mathrm{~Hz}), 2.36-2.46(1 \mathrm{H}, \mathrm{m}), 3.56-3.63(1 \mathrm{H}, \mathrm{m}), 2.86$ and $2.92(2 \times 1 \mathrm{H}$, $2 \times \mathrm{d}, J=17.0 \mathrm{~Hz}), 3.06(1 \mathrm{H}, \mathrm{dd}, J=12.5,7.5 \mathrm{~Hz}), 3.12(1 \mathrm{H}, \mathrm{dd}, J=12.5,7.0 \mathrm{~Hz}), 3.55(1 \mathrm{H}, \mathrm{dd}, J=$ 12.0, $5.0 \mathrm{~Hz}), 3.83(1 \mathrm{H}, \mathrm{dd}, J=12.0,7.0 \mathrm{~Hz}), 7.12-7.32(10 \mathrm{H}, \mathrm{m}) ; \delta_{\mathrm{C}}\left(100 \mathrm{MHz}, \mathrm{CDCl}_{3}\right) 17.8,31.0$, $35.5,42.2,49.2,53.7,56.8,106.2,126.9,127.1,128.1,128.2,128.4,128.5,131.7,142.0,143.2,170.0$; HRMS (ESI ${ }^{+}$) found 381.1679, $\mathrm{C}_{22} \mathrm{H}_{24} \mathrm{~N}_{4} \mathrm{ONa}\left(\mathrm{MNa}^{+}\right)$requires 381.1686. $\mathrm{HRMS}\left(\mathrm{ESI}^{+}\right) \mathrm{m} / z$ : $[\mathrm{M}+\mathrm{Na}]^{+}$ Calcd for $\mathrm{C}_{22} \mathrm{H}_{22} \mathrm{~N}_{4} \mathrm{NaO} 381.1686$; Found 381.1679.

\section{7,7-Diphenyl-6-(prop-1-en-2-yl)-1-azabicyclo[4.2.0]octan-8-one (38i)}

Application of the general procedure described above (for 37a) afforded lactam 37i (24 mg, 35\% from azide 22i) as a white solid. M.p $105-107^{\circ} \mathrm{C} ; \mathrm{R}_{f} 0.41$ (petrol/ether, $\left.2: 1\right)$; $v_{\max } 1746 \mathrm{~s} ; \delta_{\mathrm{H}}\left(500 \mathrm{MHz}, \mathrm{C}_{6} \mathrm{D}_{6}\right)$ 0.81-1.14 (4 H, m), $1.22(1 \mathrm{H}, \mathrm{dt}, J=13.0,3.0 \mathrm{~Hz}), 1.27(3 \mathrm{H}, \mathrm{s}), 1.92(1 \mathrm{H}, \mathrm{d}, J=12.5 \mathrm{~Hz}), 2.43(1 \mathrm{H}$, br s), $3.86(1 \mathrm{H}, \mathrm{dd}, J=13.5,6.0 \mathrm{~Hz}), 4.77$ (1 H, br s), 4.80 (1 H, br s), 6.93-6.96 (1 H, m), 7.00-7.04 (2 H, m), $7.08(1 \mathrm{H}$, app. t, $J=7.5 \mathrm{~Hz}), 7.21(2 \mathrm{H}$, app. t, $J=7.5 \mathrm{~Hz}), 7.50\left(2 \mathrm{H}\right.$, br s), $7.91\left(2 \mathrm{H}\right.$, br s); $\delta_{\mathrm{C}}$ (125 MHz, $\left.\mathrm{C}_{6} \mathrm{D}_{6}\right)$ 20.3, 20.4, 24.7, 33.9, 37.5, 70.3, 77.2, 116.4, 127.1 (2 peaks), 128.4, 128.6, 129.2, 138.8, 139.6, 145.8, 168.9 [one $\mathrm{Ph}$ resonance obscured]; HRMS $\left(\mathrm{ESI}^{+}\right) \mathrm{m} / z:[\mathrm{M}+\mathrm{Na}]^{+} \mathrm{Calcd}$ for $\mathrm{C}_{22} \mathrm{H}_{23} \mathrm{NNaO} 340.1672$; Found 340.1661.

\section{2-Bromo-1-(5-cyclohexenyl-2,3-dihydro-1H-pyrrol-1-yl)-2-methylpropan-1-one (40)}


To a $14 \mathrm{~mL}$ sample vial was added cyclic imine $\mathbf{2 3 d}(640 \mathrm{mg}, 4.29 \mathrm{mmol})$, toluene (10 mL), and $4 \AA$ molecular sieves $(1.0 \mathrm{~g})$, and the mixture was stirred under argon at $0{ }^{\circ} \mathrm{C}$. Pyridine $(380 \mu \mathrm{L}, 4.70 \mathrm{mmol})$ and $\alpha$-bromoisobutyryl bromide $(550 \mu \mathrm{L}, 4.45 \mathrm{mmol})$ were added to the solution, which became turgid and immobile before regaining mobility after $5 \mathrm{~min}$. After $10 \mathrm{~min}$, the solution was warmed to RT and stirred for a further $1 \mathrm{~h}$. The reaction mixture was filtered through a sintered glass funnel, washing through with petrol, and the filtrate was concentrated under vacuum to a toluene solution. The solution was quickly purified by silica gel chromatography, eluting with a gradient of $0.5 \%$ triethylamine/petrol to $0.5 \%$ triethylamine $/ 20 \%$ ether/petrol, yielding the title compound as a white solid (1.16 g, 91\%). M.p $89-91{ }^{\circ} \mathrm{C} ; \mathrm{R}_{f} 0.65$ (ether); $v_{\max } 1643 \mathrm{~s}, 1624 \mathrm{~s}, 1388 \mathrm{~s} ; \delta_{\mathrm{H}}\left(400 \mathrm{MHz}, \mathrm{C}_{6} \mathrm{D}_{6}\right) 1.55(2 \mathrm{H}, \mathrm{dddd}, J=12.5,6.0$, 2.5, $2.5 \mathrm{~Hz}), 1.70(2 \mathrm{H}$, dddd, $J=12.0,6.0,3.0,3.0 \mathrm{~Hz}), 1.78(6 \mathrm{H}, \mathrm{s}), 2.00(2 \mathrm{H}, \mathrm{td}, J=7.5,3.0 \mathrm{~Hz})$, 2.03-2.07 (2 H, m), 2.17-2.22 (2 H, m), $3.86(2 \mathrm{H}, \mathrm{t}, J=8.0 \mathrm{~Hz}), 5.07(1 \mathrm{H}, \mathrm{t}, J=3.0 \mathrm{~Hz}), 5.83(1 \mathrm{H}$, app. sept, $J=2.0 \mathrm{~Hz}) ; \delta_{\mathrm{C}}\left(100 \mathrm{MHz}, \mathrm{C}_{6} \mathrm{D}_{6}\right) 22.6,22.9,25.7,27.7,29.4,32.2,52.0,59.2,111.4,124.2$, 132.6, 149.7, 168.4; HRMS (ESI $\left.{ }^{+}\right)$m/z: $[\mathrm{M}+\mathrm{H}]^{+}$Calcd for $\mathrm{C}_{14} \mathrm{H}_{21}{ }^{79} \mathrm{BrNO} 298.0801$; Found 298.0800.

\section{6,6-Dimethyl-2,3,6,6a,7,8,9,10-octahydropyrrolo[2,1-a]isoquinolin-5(1H)-one (41) and 6,6-dimethyl- 2,6,6a,7,8,9,10,10a-octahydropyrrolo[2,1-a]isoquinolin-5(3H)-one (42)}

To an oven-dried two-neck $100 \mathrm{~mL}$ round-bottomed flask was added a solution of bromoester $\mathbf{4 0}$ (200 $\mathrm{mg}, 0.671 \mathrm{mmol})$ in degassed toluene $(27 \mathrm{~mL})$, and the solution was heated to $100{ }^{\circ} \mathrm{C}$. A solution of tributyltin hydride $(0.235 \mathrm{~mL}, 0.873 \mathrm{mmol})$ and AIBN $(11.0 \mathrm{mg}, 67.0 \mu \mathrm{mol})$ in degassed toluene $(20$ $\mathrm{mL}$ ) was added dropwise over $1 \mathrm{~h}$, and the mixture was heated at reflux for $22 \mathrm{~h}$. The flask was removed from the oil bath and stirred with saturated aqueous $\mathrm{KF}$ solution $(40 \mathrm{~mL})$ for $3 \mathrm{~h}$. The mixture was extracted with dichloromethane $(3 \times 20 \mathrm{~mL})$ and the combined organic extracts were dried $\left(\mathrm{MgSO}_{4}\right)$ and concentrated to yield a solid that was purified over silica gel, eluting with a gradient of $0.5 \%$ triethylamine/petrol to $0.5 \%$ triethylamine/20\% ether/petrol. The isomeric products $\mathbf{4 1}$ and $\mathbf{4 2}$ were 
isolated as a yellow oil in a ratio of 2.3:1 (106 $\mathrm{mg}, 72 \%)$. Partial separation of the isomers was achieved by further chromatography for characterization. Data for 41: $\mathrm{R}_{f} 0.40$ (ether/petrol, 2:1); $v_{\max } 1658 \mathrm{~s}$, $1641 \mathrm{~s}, 1408 \mathrm{~s} ; \delta_{\mathrm{H}}\left(500 \mathrm{MHz}, \mathrm{C}_{6} \mathrm{D}_{6}\right)$ 0.94-1.03 (1 H, m), 1.05-1.11 (2 H, m), 1.17 (3 H, s), 1.25 (2 H, app. quin, $J=7.0 \mathrm{~Hz}), 1.32(3 \mathrm{H}, \mathrm{s}), 1.54-1.67(3 \mathrm{H}, \mathrm{m}), 1.74(1 \mathrm{H}, \mathrm{tdq}, J=14.0,5.0,1.5 \mathrm{~Hz}), 1.82(1 \mathrm{H}$, app. dsext, $J=12.0,2.0 \mathrm{~Hz}), 1.95-2.09(2 \mathrm{H}, \mathrm{m}), 2.13-2.18(1 \mathrm{H}, \mathrm{m}), 3.47$ and $3.55(2 \times 1 \mathrm{H}, 2 \times \mathrm{dt}, J=11.5$, 7.0 Hz); $\delta_{\mathrm{C}}\left(125 \mathrm{MHz}, \mathrm{C}_{6} \mathrm{D}_{6}\right)$ 20.7, 21.9, 25.7, 26.5, 27.2, 27.4, 27.9, 29.5, 41.1, 46.1, 47.9, 108.9, 130.4, 172.6; HRMS $\left(\mathrm{ESI}^{+}\right) \mathrm{m} / z$ : $[\mathrm{M}+\mathrm{H}]^{+}$Calcd for $\mathrm{C}_{14} \mathrm{H}_{22} \mathrm{NO} 220.1696$; Found 220.1693. Partial data for 42 (that was not obtained free from 41): $\mathrm{R}_{f} 0.39$ (ether/petrol, $\left.2: 1\right) ; \delta_{\mathrm{H}}\left(500 \mathrm{MHz}, \mathrm{C}_{6} \mathrm{D}_{6}\right) 1.01$ and $1.32(2 \times$ $3 \mathrm{H}, 2 \times \mathrm{s}), 3.73(1 \mathrm{H}, \mathrm{ddd}, J=12.5,11.5,7.5 \mathrm{~Hz}), 3.87(1 \mathrm{H}, \mathrm{ddd}, J=12.5,11.0,6.5 \mathrm{~Hz}), 4.43(1 \mathrm{H}$, app. q, $J=2.5 \mathrm{~Hz})$.

\section{1-Bromo-6,6-dimethyl-2,3,6,6a, 7,8,9,10-octahydropyrrolo[2,1-a]isoquinolin-5(1H)-one (43)}

To a $7 \mathrm{~mL}$ sample vial was added bromoester 40 (100 mg, $0.335 \mathrm{mmol}), \mathrm{CuBr}(15.0 \mathrm{mg}, 0.105 \mathrm{mmol})$, and degassed dichloromethane $(2.5 \mathrm{~mL})$, and the insoluble mixture was stirred at RT under argon. Pentamethyldiethylenetriamine (PMDTA) $(21 \mu \mathrm{L}, 0.101 \mathrm{mmol})$ was added to the mixture, which which instantly turned a clear green color that darkened over $15 \mathrm{~min}$. The reaction mixture was filtered through a pipette of silica gel and concentrated to yield a pale yellow oil, a 1:1.3 mixture of inseparable diastereoisomers (90 mg, 90\%). The product was unstable to column chromatography with either silica gel or alumina due to the lability of the allylic bromide functionality towards dehydrobromination leading to diene 44. $\mathrm{R}_{f} 0.24$ (petrol/ether, 2:1); $v_{\max } 1605 \mathrm{~s} ; \delta_{\mathrm{H}}\left(400 \mathrm{MHz}, \mathrm{CDCl}_{3}\right) 1.01,1.04,1.14$ and $1.17(4 \times$ $\sim 1.5 \mathrm{H}, 4 \times \mathrm{s}), 1.07-1.46(\sim 3 \mathrm{H}, \mathrm{m}), 1.69-1.83(\sim 2 \mathrm{H}, \mathrm{m}), 1.87-2.04(\sim 3 \mathrm{H}, \mathrm{m}), 2.19-2.32(\sim 2 \mathrm{H}, \mathrm{m})$, $2.53(\sim 1 \mathrm{H}$, app. t, $J=7.0 \mathrm{~Hz}), 3.61-3.75(\sim 1 \mathrm{H}, \mathrm{m}), 3.90-4.06(\sim 1 \mathrm{H}, \mathrm{m}), 5.12(\sim 0.5 \mathrm{H}, \mathrm{d}, J=4.5 \mathrm{~Hz})$, $5.18(\sim 0.5 \mathrm{H}, \mathrm{d}, J=5.0 \mathrm{~Hz}) ; \delta_{\mathrm{C}}\left(100 \mathrm{MHz}, \mathrm{CDCl}_{3}\right)$ 19.9, 20.4, 25.4, 25.6, 25.7, 25.9, 26.0, 27.2 (two 
peaks), 27.9, 29.2, 29.8, 34.6, 34.7, 40.6, 40.9, 43.7, 43.8, 47.0, 47.1, 47.8, 48.0, 117.0, 117.3, 131.3, 131.6, 173.0, 173.3; HRMS (ESI $\left.{ }^{+}\right) m / z:[\mathrm{M}+\mathrm{H}]^{+}$Calcd for $\mathrm{C}_{14} \mathrm{H}_{21}{ }^{79} \mathrm{BrNO} 298.0801$; Found 298.0796.

\section{6,6-Dimethyl-2,3,7,8,9,10-hexahydropyrrolo[2,1-a]isoquinolin-5(6H)-one (44)}

To a $10 \mathrm{~mL}$ oven-dried round-bottomed flask was added bromoester $\mathbf{4 0}$ (100 mg, $0.335 \mathrm{mmol}), \mathrm{CuBr}$ (53.0 $\mathrm{mg}, 0.369 \mathrm{mmol})$, and degassed dichloromethane $(2.5 \mathrm{~mL})$, and the insoluble mixture was stirred at RT under argon. Tetramethylethylenediamine (TMEDA) $(56 \mu \mathrm{L}, 0.37 \mathrm{mmol})$ was added to the mixture, which instantly became clear and colorless; after 10 min further stirring, a green tint appeared in the solution. The reaction mixture was heated to reflux for $2.5 \mathrm{~h}$, during which the mixture became brown; the cooled reaction mixture was filtered through a plug of silica gel and concentrated to yield an oil. The oil was purified over basic alumina, eluting with a gradient of $2.5 \%$ triethylamine/petrol to $2.5 \%$ triethylamine $/ 40 \%$ ether/petrol, separating recovered starting material (40) from the product, a yellow oil (56 mg, 77\%; 92\% based on recovered 40, $16 \mathrm{mg}, 16 \%$ ). $\mathrm{R}_{f} 0.43$ (ether); $v_{\max } 1720 \mathrm{~m}, 1666 \mathrm{~s} ; \delta_{\mathrm{H}}(400$ $\left.\mathrm{MHz}, \mathrm{C}_{6} \mathrm{D}_{6}\right) 1.36-1.40(4 \mathrm{H}, \mathrm{m})$ overlaying $1.35(6 \mathrm{H}, \mathrm{s}), 1.75-1.81(2 \mathrm{H}, \mathrm{m}), 1.96-2.01(2 \mathrm{H}, \mathrm{m}), 2.09-$ $2.15(2 \mathrm{H}, \mathrm{m}), 3.80(2 \mathrm{H}$, app. t, $J=9.0 \mathrm{~Hz}), 4.56(1 \mathrm{H}, \mathrm{t}, J=3.0 \mathrm{~Hz}) ; \delta_{\mathrm{C}}\left(100 \mathrm{MHz}, \mathrm{C}_{6} \mathrm{D}_{6}\right) 22.3,23.1$,

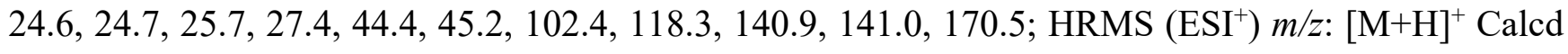
for $\mathrm{C}_{14} \mathrm{H}_{20} \mathrm{NO} 218.1539$; Found 298.1538.

\section{6,6-Dimethyl-2,3,6,8,9,10-hexahydropyrrolo[2,1-a]isoquinolin-5(1H)-one (45)}

When tricyclic lactam $\mathbf{4 4}$ was purified on silica gel in the absence of triethylamine, or when dissolved in $\mathrm{CDCl}_{3}$, partial isomerization occurred to give lactam 45 in $\sim 1: 1$ ratio with $\mathbf{4 4}$; this isomer was isolated by chromatography for characterization. $\mathrm{R}_{f} 0.33$ (ether); $v_{\max } 1660 \mathrm{~s}, 1410 \mathrm{~m} ; \delta_{\mathrm{H}}\left(500 \mathrm{MHz}, \mathrm{C}_{6} \mathrm{D}_{6}\right) 1.19$ (2 H, app. quin, $J=7.0 \mathrm{~Hz}), 1.51(6 \mathrm{H}, \mathrm{s}), 1.57(2 \mathrm{H}$, app. quin, $J=6.0 \mathrm{~Hz}), 1.99-2.05(4 \mathrm{H}, \mathrm{m}), 2.11(2$ $\mathrm{H}, \mathrm{tt}, J=6.5,1.0 \mathrm{~Hz}), 3.47(2 \mathrm{H}, \mathrm{t}, J=7.0 \mathrm{~Hz}), 5.44(1 \mathrm{H}, \mathrm{t}, J=4.0 \mathrm{~Hz}) ; \delta_{\mathrm{C}}\left(125 \mathrm{MHz}, \mathrm{C}_{6} \mathrm{D}_{6}\right) 21.5,22.9$, 


\author{
26.1, 26.3, 27.6, 27.8, 44.1, 46.3, 119.3, 124.1, 131.9, 141.5, 172.9; HRMS (ESI $\left.{ }^{+}\right) m / z:[\mathrm{M}+\mathrm{Na}]^{+}$Calcd \\ for $\mathrm{C}_{14} \mathrm{H}_{19} \mathrm{NNaO} 240.1359$; Found 240.1358.
}

\title{
ASSOCIATED CONTENT
}

\section{Supporting information}

The Supporting Information is available free of charge on the ACS Publications website at DOI: 10.1021/acs.joc.@@@@@@.

Reaction schemes for preparation of substrates, and copies of ${ }^{1} \mathrm{H}$ and ${ }^{13} \mathrm{C}$ NMR spectra.

\section{AUTHOR INFORMATION \\ Corresponding author \\ *E-mail: jeremy.robertson@chem.ox.ac.uk \\ ORCID \\ John T. R. Liddon: 0000-0002-5287-9817 \\ Peter J. Lindsay-Scott: 0000-0002-4721-3594 \\ Jeremy Robertson: 0000-0002-6809-8265}

\section{Notes}

The authors declare no competing financial interest.

\section{ACKNOWLEDGMENTS}

The authors are grateful to the Engineering and Physical Sciences Research Council, UK, and Eli Lilly for a studentship (to J.T.R.L.), and Prof. T. D. W. Claridge, University of Oxford, and his staff for NMR services.

\section{REFERENCES}

(1) Creary, X.; Wang, Y.-X.; Jiang, Z. $\alpha$-Imino and $\alpha$-Oximino Carbocations. A Comparison with $\alpha$-Carbonyl and $\alpha$-Thiocarbonyl Carbocations. J. Am. Chem. Soc. 1995, 117, 3044-3053.

(2) Zhurakovskyi, O.; Ellis, S. R.; Thompson, A. L.; Robertson, J. Access to a Guanacastepene and Cortistatin-Related Skeleton via Ethynyl Lactone Ireland-Claisen Rearrangement and Transannular (4 + 3)-Cycloaddition of an Azatrimethylenemethane Diyl. Org. Lett. 2017, 19, 2174-2177.

(3) See for example: (a) Huisgen, R.; Möbius, L.; Szeimies, G. 1.3-Dipolar Cycloadditions, XIV. The Addition of Organic Azides to Enol Ethers: Orientation and Triazoline Decomposition. Chem. Ber. 1965, 98, 1138-1152; (b) Huisgen, R.; Szeimies, G. 1.3Dipolar Cycloadditions, XV. The Steric Course of the Azide Addition to Enol Ethers. Chem. Ber. 1965, 98, 1153-1158; (c) Gerlach, O.; Reiter, P. L.; Effenberger, F. Enol Ethers, XII. Reactions of Cyclopropylolefins and Enol Ethers with Organic Azides. Liebigs Ann. Chem. 1974, 1895-1907; (d) Dahl, R. S.; Finney, N. S. A Surprising Dipolar Cycloaddition Provides Ready Access to Aminoglycosides. J. Am. Chem. Soc. 2004, 126, 8356-8357. 
(4) (a) Uhle, F. C. Solvolysis of Pseudodiosgenin 27-p-Toluenesulfonate. Tetrahedron Lett. 1964, 3099-3103; (b) Uhle, F. C. Synthesis and Transformations of Heptacyclic Triazoline Derived from Pseudodiosgenin. J. Org. Chem. 1967, 32, 1596-1601; (c) Ciufolini, M. A.; Chen, M.; Lovett, D. P.; Deaton, M. V. Application of Ene-Like Reactions of Aldehydes with Vinyl Ethers: Facile Assembly of Benzacocenone Intermediates for Mitomycinoids. Tetrahedron Lett. 1997, 38, 4355-4358; (d) Yao, L.; Smith, B. T.; Aubé, J. Base-Promoted Reactions of Bridged Ketones and 1,3- and 1,4-Haloalkyl Azides: Competitive Alkylation vs Azidation Reactions of Ketone Enolates. J. Org. Chem. 2004, 69, 1720-1722; (e) Zha, X.; Sun, H.; Hao, J.; Zhang, Y. Efficient Synthesis of Solasodine, O-Acetylsolasodine, and Soladulcidine as Anticancer Steroidal Alkaloids. Chem. Biodivers. 2007, 4, 25-31; (f) Zha, X. M.; Zhang, F. R.; Shan, J. Q.; Zhang, Y. H.; Liu, J. O.; Sun, H. B. Synthesis and Evaluation of in vitro Anticancer Activity of Novel Solasodine Derivatives. Chin. Chem. Lett. 2010, 21, 1087-1090.

(5) The same stucture produced from imidate + diazoalkane cycloaddition: Hamdi, N.; Fischmeister, C.; Dixneuf, P. H.; Romerosa Nievas, A. Regioselective Synthesis of a New [1,2,3]-Triazoles Directly from Imidates. J. Heterocyclic Chem. 2006, 43, 499-501.

(6) (a) Schöllkopf, U.; Hänßle, P. 1-Ethoxyvinylllithium as a Reagent for Nucleophilc Acetylation. Liebigs Ann. Chem. 1972, 763, 208-210; (b) Baldwin, J. E.; Höfle, G. A.; Lever, Jr., O. W. $\alpha$-Methoxyvinyllithium and Related Enol Ethers. Practical Reagents for Nucleophilic Acylation. J. Am. Chem. Soc. 1974, 96, 7125-7127; (c) Boeckman, Jr., R. K.; Bruza, K. J. Cyclic Vinyl Ether Carbanions I: Synthetic Equivalents of $\beta$-Acylvinyl and Substituted Acyl Anions. Tetrahedron Lett. 1977, 4187-4190.

(7) Jones, K. O.; Houk, K. N. Predictions of Substituent Effects in Thermal Azide 1,3-Dipolar Cycloadditions: Implications for Dynamic Combinatorial (Reversible) and Click (Irreversible) Chemistry. J. Org. Chem. 2008, 73, 1333-1342.

(8) Huisgen, R.; Szeimies, G.; Möbius, L. 1,3-Dipolar Cycloadditions, XXXII. Kinetics of the Addition of Organic Azides to CCMultiple Bonds. Chem. Ber. 1967, 100, 2494-2507.

(9) Xiao, Y.; Zhang, L. Synthesis of Bicyclic Imidazoles via [2 + 3] Cycloaddition between Nitriles and Regioselectively Generated $\alpha$-Imino Gold Carbene Intermediates. Org. Lett. 2012, 14, 4662-4665.

(10) Montevecchi, P. C.; Navacchia, M. L.; Spagnolo, P. A Study of Vinyl Radical Cyclization onto the Azido Group by Addition of Sulfanyl, Stannyl, and Silyl Radicals to Alkynyl Azides. Eur. J. Org. Chem. 1998, 1219.

(11) Mancuso, L.; Knobloch, T.; Bucholz, J.; Hartwig, J.; Möller, L.; Seidel, K.; Collisi, W.; Sasse, F.; Kirschning, A. Preparation of Thermocleavable Conjugates Based on Ansamitocin and Superparamagnetic Nanostructured Particles by a Chemobiosynthetic Approach. Chem. Eur. J. 2014, 20, 17541-17551.

(12) Bao, R.; Valverde, S.; Herradón, B. The Claisen-Johnson Rearrangement Route to 1,4-Dicarbonyl Compounds: Synthesis of Ethyl 4-Ethoxy-4-alkenoates as Masked 4-Oxo Esters. Synlett 1992, 217-218.

(13) Cf. Doi, T.; Robertson, J.; Stork, G.; Yamashita, A. Regiospecificity in the Cyclization of 6-(1-Hydroxyalkyl) Geraniol Derivatives. A Simple Route to the Taxol A-Ring System. Tetrahedron Lett. 1994, 35, 1481-1484.

(14) For discussion, see for example: (a) Wu, L.; Chen, Y.; Luo, J.; Sun, Q.; Peng, M.; Lin, Q. Base-mediated Reaction of Vinyl Bromides with Aryl Azides: One-pot Synthesis of 1,5-Disubstituted 1,2,3-Triazoles. Tetrahedron Lett. 2014, 55, 3847-3850; (b) Raj, J. P.; Gangaprasad, D.; Vajjiravel, M.; Karthikeyan, K.; Elangovan, J. CuO-Nanoparticales Catalyzed Synthesis of 1,4Disubstituted-1,2,3-Triazoles from Bromoalkenes. J. Chem. Sci. 2018, 130:44 (6 pages). 
(15) See for example Ref. 7 or Reichardt, C. Solvents and Solvent Effects in Organic Chemistry. Wiley-VCH, Weinheim, 2003, pp. 191-192.

(16) Brown, H. C.; Ichikawa, K. Chemical Effects of Steric Strains - XIV. The Effect of Ring Size on the Rate of Reaction of the Cyclanones with Sodium Borohydride. Tetrahedron 1957, 1, 221-230.

(17) Allinger, N. L.; Zalkow, V. Conformational Analysis. IX. The gem-Dimethyl Effect. J. Org. Chem. 1960, 25, 701-704.

(18) Roth, M.; Dubs, P.; Götschi, E.; Eschenmoser, A. Sulfide Contraction via Alkylative Coupling: Method for Preparation of $\beta$-Dicarbonyl Derivatives. Helv. Chim. Acta 1971, 54, 710-734.

(19) (a) Hegde, S. G.; Beckwith, D.; Doti, R.; Wolinsky, J. Synthesis with HOCl. Conversion of Pulegone and Isopulegol to Menthofuran. Preparation of 3,6-Dimethyl-2,6-cycloheptadien-1-one from Phorone. J. Org. Chem. 1985, 50, 894-896; (b) Toum, V.; Kadouri-Puchot, C.; Hamon, L.; Lhommet, G.; Mouriès-Mansuy, V.; Vanucci-Bacqué, C.; Dechoux, L. NBromosuccinimide-Promoted Cyclization of $\beta$-Carboxymethyl Enamino Esters; Synthesis of Functionalized 4-Amino-2(5H)Furanones. Synthesis 2011, 2781-2788.

(20) Niwa, H.; Miyachi, Y.; Okamoto, O.; Uosaki, Y.; Yamada, K. Total Synthesis of Optically Active Integerrimine, a TwelveMembered Dilactonic Pyrrolizidine Alkaloid of Retronecine Type. II. Enantioselective Synthesis of (+)-Retronecine. Tetrahedron Lett. 1986, 27, 4605-4608.

(21) Morimoto, T.; Chatani, N.; Murai, S. The First Catalytic Carbonylative [4 + 1] Cycloaddition Using a 1,3-Conjugated System. A New Transformation of $\alpha, \beta$-Unsaturated Imines to Unsaturated $\gamma$-Lactams Catalyzed by $\mathrm{Ru}_{3}(\mathrm{CO})_{12}$. J. Am. Chem. Soc. 1999, 121, 1758-1759.

(22) Hussain, S.; Leipold, F.; Man, H.; Wells, E.; France, S. P.; Mulholland, K. R.; Grogan, G.; Turner, N. J. An (R)-Imine Reductase Biocatalyst for the Asymmetric Reduction of Cyclic Imines. ChemCatChem 2015, 7, 579-583.

(23) (a) Jayakumar, S.; Ishar, M. P. S.; Mahajan, M. P. Recent Advances in Synthetic Applications of Azadienes. Tetrahedron 2002, 58, 379-471; (b) Groenendaal, B.; Ruijter, E.; Orru, R. V. A. 1-Azadienes in Cycloaddition and Multicomponent Reactions Towards N-Heterocycles. Chem. Commun. 2008, 5474-5489; (c) Monbaliu, J.-C. M.; Masschelein, K. G. R.; Stevens, C. V. Electron-deficient 1- and 2-Azabuta-1,3-dienes: a Comprehensive Survey of their Synthesis and Reactivity. Chem. Soc. Rev. 2011, 40, 4708-4739.

(24) Liddon, J. T. R. The Versatile Chemistry of Azidoalkyl Enol Ethers and their Equivalents. D.Phil. Thesis, Oxford, 2015.

(25) Michael, J. P. Simple Indolizidine and Quinolizidine Alkaloids. In The Alkaloids: Chemistry and Biology. Knölker, H.-J., Ed.; Academic Press: 2016, Vol. 75, pp. 1-498.

(26) Smith, L. I.; Hoehn, H. H. Diphenylketene. Org. Synth. 1955, Coll. Vol. III, 356-357.

(27) (a) Brady, W. T.; Shieh, C. H. Halogenated Ketenes. 38. Cycloaddition of $\alpha, \beta$-Unsaturated Imines with Ketenes to Yield Both $\beta$ - and $\delta$-Lactams. J. Org. Chem. 1983, 48, 2499-2502; (b) Saito, T.; Kobayashi, S.; Ohgaki, M.; Wada, M.; Nagahiro, C. Diene-transmissive Hetero Diels-Alder Reaction of Cross-conjugated Azatrienes with Ketenes: a Novel and Efficient, Stereocontrolled Synthetic Method for Hexahydroquinolinones. Tetrahedron Lett. 2002, 43, 2627-2631; (c) Kobayashi, S.; Semba, T.; Takahashi, T.; Yoshida, S.; Dai, K.; Otani, T.; Saito, T. A Novel and Efficient Stereo-controlled Synthesis of 
Hexahydroquinolinones via the Diene-transmissive Hetero-Diels-Alder Reaction of Cross-conjugated Azatrienes with Ketenes and Electrophilic Dienophiles. Tetrahedron 2009, 65, 920-933.

(28) Kraus, G. A.; Neuenschwander, K. A Facile Synthesis of N-Acyl-2-pyrrolines. J. Org. Chem. 1981, 46, 4791-4792.

(29) Clark, A. J. Copper Catalyzed Atom Transfer Radical Cyclization Reactions. Eur. J. Org. Chem. 2016, 2231-2243.

(30) De Campo, F.; Lastécouères, D.; Verlhac, J.-B. New Copper(I) and Iron(II) Complexes for Atom Transfer Radical Macrocyclisation Reactions. J. Chem. Soc., Perkin Trans. 1 2000, 575-580.

(31) Benedetti, M.; Forti, L.; Ghelfi, F.; Pagnoni, U. M.; Ronzoni, R. Halogen Atom Transfer Radical Cyclization of N-Allyl- $N$ Benzyl-2,2-Dihaloamides to 2-Pyrrolidinones, Promoted by $\mathrm{Fe}^{0}-\mathrm{FeCl}_{3}$ or $\mathrm{CuCl}-\mathrm{TMEDA}$. Tetrahedron 1997, 53, 14031-14042.

(32) Boeckman, Jr., R. K.; Tagat, J. R.; Johnston, B. H. The Chemistry of Cyclic Vinyl Ethers 4: Cyclization of Unsaturated Cyclic Vinyl Ether Epoxides. Heterocycles 1987, 25, 33-41.

(33) Patra, D.; Ghosh, S. Photocycloaddition-cyclobutane Rearrangement to Spirocyclopentanones: Application in a Formal Synthesis of ( \pm )- $\alpha$-Cedrene. J. Chem. Soc. Perkin Trans. 1 1995, 2635-2641.

(34) Wills, V. S.; Metzger, J. I.; Allen, C.; Varney, M. L.; Wiemer, D. F.; Holstein, S. A. Bishomoisoprenoid Triazole Bisphosphonates as Inhibitors of Geranylgeranyl Diphosphate Synthase. Bioorg. Med. Chem. 2017, 25, 2437-2444.

(35) Phipps, R. J.; McMurray, L.; Ritter, S.; Duong, H. A.; Gaunt, M. J. Copper-Catalyzed Alkene Arylation with Diaryliodonium Salts. J. Am. Chem. Soc. 2012, 134, 10773-10776.

(36) Albrecht, U.; Langer, P. Synthesis of Spirocyclic Butenolides by Ring Closing Metathesis. Tetrahedron 2007, 63, 46484654.

(37) Chen, L.; Gill, G. B.; Pattenden, G.; Simonian, H. Consecutive 6-Endo-Trigonal Cyclisations from Polyene Acyl Radical Intermediates Leading to Decalone and Perhydrophenanthrone Ring Constructions. J. Chem. Soc. Perkin Trans. 1 1996, 3143.

(38) Han, S.-J.; Doi, R.; Stoltz, B. M. Nickel-Catalyzed Intramolecular C-O Bond Formation: Synthesis of Cyclic Enol Ethers. Angew. Chem. Int. Ed. 2016, 55, 7437-7440.

(39) Hodgson, D. M.; Chung, Y. K.; Nuzzo, I.; Freixas, G.; Kulikiewicz, K. K.; Cleator, E.; Paris, J.-M. Intramolecular Cyclopropanation of Unsaturated Terminal Epoxides and Chlorohydrins. J. Am. Chem. Soc. 2007, 129, 4456-4462.

(40) Vang, T.; Xie, Y.; Liu, W. H.; Vidovic, D.; Liu, Y.; Wu, S.; Smith, D. H.; Rinderspacher, A.; Chung, C.; Gong, G.; Mustelin, T.; Landry, D. W.; Rickert, R. C.; Schürer, S. C.; Deng, S.-X.; Tautz, L. Inhibition of Tyrosine Phosphatase by Benzofuran Salicylic Acids. J. Med. Chem. 2011, 54, 562-571.

(41) Data obtained by T. A. Clohessy, Preparation and Reactions of $\omega$-Azidoalkenes. Chemistry Part II Thesis, Oxford, 2013.

(42) Green, S. A.; Vásquez-Céspedes, S.; Shenvi, R. A. Iron-Nickel Dual-Catalysis: A New Engine for Olefin Functionalization and the Formation of Quaternary Centers. J. Am. Chem. Soc. 2018, 140, 11317-11324.

(43) Ono, N.; Kamimura, A.; Miyake, H.; Hamamoto, I.; Kaji, A. New Synthetic Methods. Conjugate Addition of Alkyl Groups to Electron Deficient Olefins with Nitroalkanes as Alkyl Anion Equivalents. J. Org. Chem. 1985, 50, 3692-3698.

(44) Morimoto, T.; Chatani, N.; Murai, S. The First Catalytic Carbonylative [4 + 1] Cycloaddition Using a 1,3-Conjugated System. A New Transformation of $\alpha, \beta$-Unsaturated Imines to Unsaturated $\gamma$-Lactams Catalyzed by $\mathrm{Ru}_{3}(\mathrm{CO})_{12}$. J. Am. Chem. Soc. 1999, 121, 1758-1759. 